\begin{abstract}
SCOFIELD, DANIEL. Patterns in Khovanov Link and Chromatic Graph Homology. (Under the direction of Radmila Sazdanović.)

In 2000, Khovanov introduced a homology theory whose graded Euler characteristic is the Jones polynomial. The rich structure of Khovanov homology contains topological information such as the Rasmussen $s$-invariant and spectral sequences that relate it to other link homology theories. Although torsion, especially $\mathbb{Z}_{2}$ torsion, frequently appears in Khovanov homology, its relations with topological properties of knots are not well understood. Using a similar construction, Helme-Guizon and Rong have categorified the chromatic polynomial for graphs with a theory known as chromatic graph homology. There is a partial isomorphism between Khovanov homology of a semi-adequate link $L$ and the chromatic homology $H_{\mathcal{A}_{2}}(G)$ of a state graph $G$ obtained from a diagram $D$ of $L$. This isomorphism allows us to describe part of Khovanov homology in terms of combinatorial graph data.

For chromatic homology over $\mathcal{A}_{2}$, we improve a bound given by Helme-Guizon and Rong by stating the precise homological span of $H_{\mathcal{A}_{2}}(G)$. We determine how $H_{\mathcal{A}_{2}}(G)$ changes under structural operations on $G$ and use these results to describe torsion in Khovanov homology for several families of alternating 3-strand pretzel links and rational 2-bridge links. We give explicit formulas for the ranks of several chromatic homology groups on the top diagonal, and use the chromatic polynomial to compute the first $n$ coefficients of the Jones polynomial for links with certain diagrams. We also define a new invariant, the girth of a link, that describes the largest possible contribution of chromatic homology to Khovanov homology. We prove some basic properties of this invariant and provide bounds on the girths of many knots and links.

When other polynomial algebras of the form $\mathcal{A}_{m}=\mathbb{Z}[x] /\left(x^{m}\right)$ are used in the construction, the resulting homologies may be stronger than the chromatic polynomial and may contain torsion of arbitrary order. In the case where $m$ is greater than 2, we provide a lower bound for the homological span of $H_{\mathcal{A}_{m}}(G)$ and prove that the homological thickness of $H_{\mathcal{A}_{m}}(G)$ is determined by $m$ and the number of vertices of $G$. We describe several examples of cochromatic graphs distinguished by chromatic homology over $\mathcal{A}_{3}$, and show that the this version of chromatic homology can distinguish graphs with the same Tutte polynomial and 2-isomorphism type.
\end{abstract}




\section{Patterns in Khovanov Link and Chromatic Graph Homology}

by

Daniel Scofield

A dissertation submitted to the Graduate Faculty of

North Carolina State University

in partial fulfillment of the

requirements for the degree of

Doctor of Philosophy

Mathematics

Raleigh, North Carolina

2018

APPROVED BY:

Radmila Sazdanović

Chair of Advisory Committee
Patricia Hersh

Nathan Reading 


\section{DEDICATION}

To my parents and my sisters, for their love, encouragement, and sense of humor. 


\section{BIOGRAPHY}

Daniel Scofield was born in Winston-Salem, North Carolina on October 6th, 1990 and spent his childhood in Washington County, Pennsylvania. He first became interested in abstract mathematics while reading about non-Euclidean geometries and the artwork of M.C. Escher in Harold Jacobs' Geometry textbook. He attended Grove City College as a mathematics major from 2009 to 2013, participating in an REU summer program at Clemson University which helped him decide to pursue graduate studies. During his first year as a graduate student at North Carolina State University, Daniel began a collaboration with Dr. Radmila Sazdanovic which eventually led to his dissertation on the relationship between certain invariants of knots and graphs. He earned an M.S. in mathematics in 2016 and plans to graduate with a Ph.D. in May 2018. 


\section{ACKNOWLEDGEMENTS}

I would like to thank my advisor, Dr. Radmila Sazdanović, for her patience and support in helping me complete my dissertation. I would also like to thank Dr. Adam Lowrance, Dr. Jozef Przytycki, Alex Chandler, and Victor Summers for many fruitful discussions, and the members of my committee for their assistance and insight.

I would never have reached this point without the help of many teachers who contributed to my education and encouraged me to pursue graduate studies in mathematics, including my parents David and Elizabeth Scofield and Drs. Eric Bancroft, Erin Bancroft, Jim Brown, Ryan Higginbottom, and Gary Thompson, along with numerous faculty members at Grove City College. Finally, I would like to express my appreciation to all the friends who have supported me over the past five years, especially Michael Bowser, Paul Miles, Paul Mosley, McKay Sullivan, and my North Raleigh church community at Redeemer PCA. 


\section{TABLE OF CONTENTS}

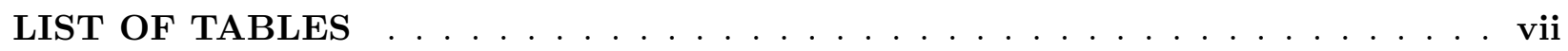

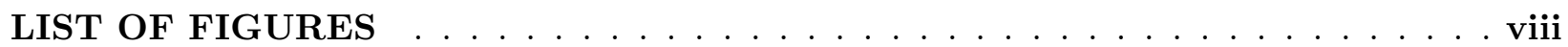

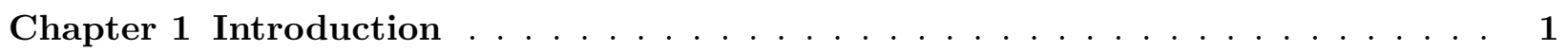

Chapter 2 Background $\ldots \ldots \ldots \ldots \ldots \ldots$

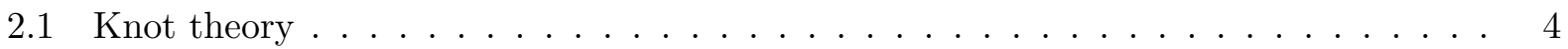

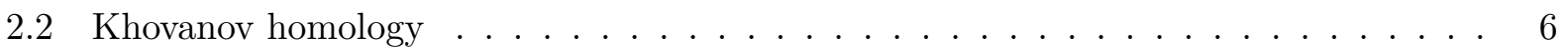

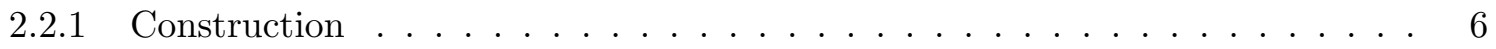

2.2.2 Supported gradings and diagonals . . . . . . . . . . . . . . . 9

2.2.3 Torsion . . . . . . . . . . . . . . . . . . . 10

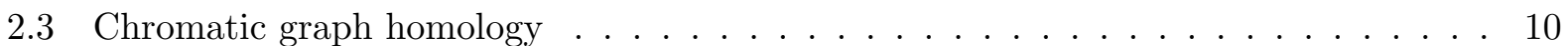

2.3 .1 Construction . . . . . . . . . . . . . . . . . . 11

2.3.2 Chromatic homology over $\mathbb{Z}[x] /\left(x^{2}\right) \ldots \ldots \ldots \ldots \ldots$

2.3.3 Chromatic homology over $\mathbb{Z}[x] /\left(x^{m}\right)$ for $m>2 \ldots \ldots \ldots \ldots$

2.4 Correspondence between Khovanov and chromatic homologies . . . . . . . . . . . 15

Chapter 3 Structure of chromatic homology over $\mathcal{A}_{2}=\mathbb{Z}[x] /\left(x^{2}\right) \ldots \ldots \ldots$

3.1 Homological span . . . . . . . . . . . . . . . . . . . . . 18

3.2 Addition of cycles . . . . . . . . . . . . . . . . . . 22

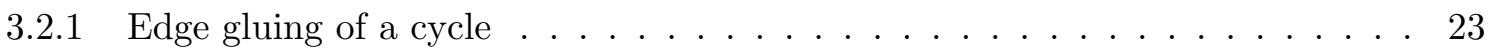

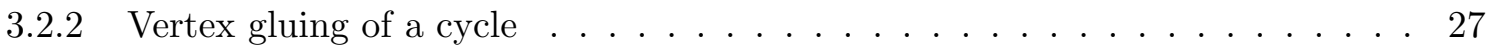

3.2.3 Khovanov homology of certain 3 -strand pretzel links . . . . . . . . . . . . 29

3.3 Existence of gaps in Khovanov and chromatic homology . . . . . . . . . . . . . . 32

3.4 Khovanov homology groups and the corresponding coefficients of the Jones polynomial 34

Chapter 4 Structure of chromatic homology over $\mathcal{A}_{m}=\mathbb{Z}[x] /\left(x^{m}\right), m>2 \ldots 43$

4.1 Width of chromatic homology over $\mathcal{A}_{m} \ldots \ldots \ldots \ldots$. . . . . . . 43

$4.2 H^{i_{\max }}(G)$ tail of homology . . . . . . . . . . . . . . . . . . 44

4.3 Relative strengths of chromatic homology and graph polynomials . . . . . . . . . 47

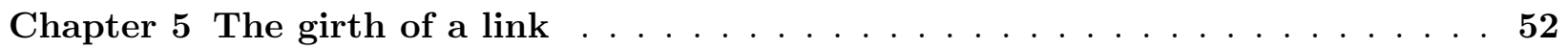

5.1 Definition of girth and properties . . . . . . . . . . . . . . . 52

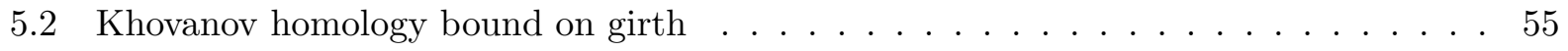

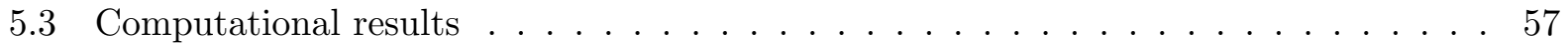

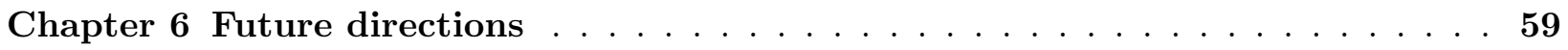

6.1 Chromatic graph homology over $\mathcal{A}_{2} \ldots \ldots \ldots \ldots \ldots \ldots$

6.2 Chromatic graph homology over $\mathcal{A}_{m}, m>2 \ldots \ldots \ldots$

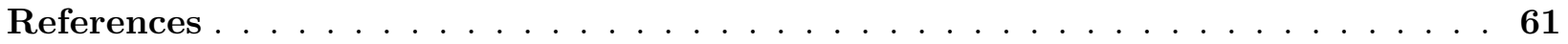




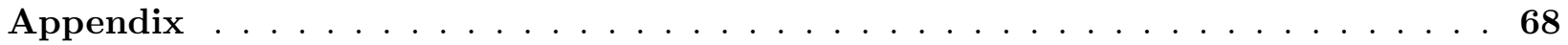

Appendix A Proofs of combinatorial identities . . . . . . . . . . . . . 69 


\section{LIST OF TABLES}

Table 3.1 Torsion in Khovanov homology of pretzel knot $L=(-3,-2,-3)$ and in chromatic homology of the corresponding graph $G=\theta(3,2,3)$. Entries in boldface denote the range where torsion is isomorphic. . . . . . . . . . . . 31

Table 4.1 Computation of $H_{\mathcal{A}_{5}}^{1, j}(G)$ in Mathematica [Sco], where $G$ is a triangle with 2 pendant edges (see Example 4). . . . . . . . . . . . . . . 46

Table 5.1 Khovanov homology of the link $L D_{4}=\overline{-(4 ; 4)(4 ; 4)}$ with boldface entries denoting matching homology with chromatic homology. . . . . . . . . . . . 57

Table 5.2 Chromatic homology of $G=G_{+}\left(D_{4}\right)=P_{4} * P_{4} * P_{4} * P_{4}$ with boldface entries denoting matching homology with chromatic homology. . . . . . . . . . 57

Table 5.3 Upper bounds for girth of alternating knots (up to 16 crossings), based on Jones polynomial coefficients. . . . . . . . . . . . . . . . . 58 


\section{LIST OF FIGURES}

Figure 2.1 The Reidemeister moves. . . . . . . . . . . . . . . . . . . 5

Figure 2.2 Positive and negative resolutions of a crossing. . . . . . . . . . . . . 5

Figure 2.3 Convention for positive/negative crossings of an oriented link diagram. . . . . . . 6

Figure 2.4 Kauffman states and Khovanov chain groups for the Hopf link $L=2_{1} \ldots \ldots$. . . 7

Figure 2.5 Subgraphs and chromatic chain groups of a 3 -cycle $G=P_{3} \ldots \ldots \ldots$. . . . . 12

Figure 2.6 Diagram of $5_{1}$ and its corresponding planar graph. . . . . . . . . . . . . . . 15

Figure 2.7 Diagram of torus knot $T(2,7)$ and its corresponding all-positive state graph. . . 16

Figure 3.1 Contraction sequence $\left\{G_{0}, G_{1}, G_{2}\right\}$ with ending with a tree $G_{2}$. . . . . . . . 19

Figure 3.2 Edge and vertex gluings of graphs. . . . . . . . . . . . . . . . . . . . . 24

Figure 3.3 Multibridge graphs (left), multibridge graph $\theta(3,2,3)$ (middle) which can be seen as gluing two pentagons along two edges, that corresponds to the standard diagram of pretzel knot $(-3,-2,-3)$ (right). . . . . . . . . . . 30

Figure 3.4 Graphs $T_{1}$ through $T_{20}$ involved in the computation of the 5 th and 6 th coefficients of the chromatic polynomial $[$ Bie05] . . . . . . . . . . . . . . 35

Figure 3.5 Graphs $T_{21}$ through $T_{31}$ involved in the computation of the 5 th and 6 th coefficients of the chromatic polynomial $[\mathrm{Bie} 05] . \ldots \ldots \ldots$

Figure 4.1 Whitney flip operation on graphs. . . . . . . . . . . . . . . . . 48

Figure 4.2 Cochromatic graphs of Marion C. Gray [PPS09, Example 6.4]. . . . . . . . . . . 49

Figure 4.3 An example of cochromatic graphs from [BM76] . . . . . . . . . . . . . . 49

Figure 4.4 First example of cochromatic graphs from [CWJ79]. . . . . . . . . . . . . . . 49

Figure 4.5 Second example of cochromatic graphs from [CWJ79] . . . . . . . . . . . 50

Figure 4.6 An example of cochromatic graphs from [KG90]. . . . . . . . . . . . . . . 50

Figure 4.7 An example of cochromatic graphs from [DKT05] . . . . . . . . . . . . . 51

Figure 4.8 Two graphs in the same 2 -isomorphism class. . . . . . . . . . . . . . 51

Figure 5.1 (a) Mirror of the link 12n888; (b) Graph $G_{+}\left(D_{3}\right)$ corresponding to diagram in (a); (c) Infinite family $D_{n}=\overline{-(n ; n)(n ; n)}$; (d) Graph $G_{+}\left(D_{n}\right)$ corresponding to diagram in $(\mathrm{c}) \ldots \ldots \ldots \ldots \ldots \ldots$ 


\section{Chapter 1}

\section{Introduction}

The Jones polynomial, first introduced in 1984, is a topological invariant of knots and links in threedimensional space. Khovanov [Kho00] introduced a homology theory for links whose graded Euler characteristic is the Jones polynomial. This theory, known as Khovanov link homology, lifts the Jones polynomial and its properties, a process known as categorification. Khovanov homology has a rich algebraic structure which contains new invariants such as the Rasmussen $s$-invariant [Ras10] and spectral sequences that relate it to other link homology theories. The Khovanov homology of many links contains $\mathbb{Z}_{2}$ torsion, with $\mathbb{Z}_{p}$ torsion appearing less frequently for odd primes $p$. However, few results are known which relate this torsion to the topological properties of a knot or link. Shumakovitch conjectured that the Khovanov homology of any link (except for disjoint unions or connect sums of unlinks and Hopf links) has torsion of order 2. This conjecture has been found true for homologically thin links, which have only $\mathbb{Z}_{2}$ torsion. On the other hand, odd torsion of many orders is possible in non-alternating links such as torus links and their deformations, which may contain odd torsion of various orders [BN02, $\left.\mathrm{MPS}^{+} 17\right]$.

The process that led from the Jones polynomial to the construction of Khovanov homology is known as categorification. Categorification takes objects with integral structure, such as natural numbers or polynomials, and builds new algebraic structures which contain all the properties of the original objects, along with (possibly) new information. Since Khovanov's seminal paper, a number of other combinatorial objects have been categorified. The chromatic polynomial for graphs has been categorified by Helme-Guizon and Rong [HGR05] using a construction similar to that of Khovanov homology. Given a semi-adequate link $L$, there is a partial isomorphism between the Khovanov homology of $L$ and the chromatic homology of the all-positive state graph obtained from a diagram $D$ of $L$. The extent of the isomorphism depends only on the length of the shortest

cycle in this graph. Lowrance and Sazdanovic [LS17] have shown that chromatic homology over the algebra $\mathcal{A}_{2}=\mathbb{Z}[x] /\left(x^{2}\right)$ has only $\mathbb{Z}_{2}$ torsion, and is equivalent to the chromatic polynomial. When other polynomial algebras of the form $\mathcal{A}_{m}=\mathbb{Z}[x] /\left(x^{m}\right)$ are used in the construction, the resulting 
homologies may be stronger than the chromatic polynomial and may contain torsion of arbitrary order.

In Chapter 3, we use graph-theoretic results and the partial isomorphism with chromatic homology to provide a combinatorial interpretation for certain properties in Khovanov homology. We improve the bound given by Helme-Guizon and Rong for the homological span of chromatic homology over $\mathcal{A}_{2}$, stating the precise homological span in terms of the numbers of vertices and blocks in a given graph. We determine how chromatic homology over $\mathcal{A}_{2}$ changes under structural operations on $G$, and give explicit formulas for the ranks of several chromatic homology groups. We also use the chromatic polynomial to compute the first $n$ coefficients of the Jones polynomial for links with certain diagrams, along with the ranks and torsion of the corresponding groups in Khovanov homology.

In Chapter 4, we provide a lower bound for the homological span of $H_{\mathcal{A}_{m}}(G), m>2$ and prove that the homological thickness of $H_{\mathcal{A}_{m}}(G)$ is determined by $m$ and the number of vertices of $G$. We describe some interesting patterns in the last homological grading of $H_{\mathcal{A}_{m}}(G)$ and offer a conjecture on how this grading relates to the addition of new edges in $G$. We describe several examples of cochromatic graphs distinguished by chromatic homology over $\mathcal{A}_{3}$ which exhibit different properties from the example described in [PPS09]. In addition, we show that this version of chromatic homology can distinguish graphs which have the same Tutte polynomial as well as the same 2-isomorphism type.

In Chapter 5, we define the girth of a link to be the maximum girth of any all-positive state graph associated to a diagram of the link. We determine the girth of $(2, n)$ torus links, and provide lower bounds on the girths of alternating pretzel links and connected sums of knots. We show that for any link $L$ whose Khovanov homology lies on two diagonals, the coefficients of the Jones polynomial provide an upper bound on girth, and we provide statistics on this upper bound for alternating knots with 16 or fewer crossings. 


\section{Chapter 2}

\section{Background}

Categorification is a process which lifts numbers or polynomials to objects in a category. For example, the set of natural numbers $\mathbb{N}$ may be thought of as a "shadow" of the category of finitedimensional vector spaces. For each integer $n$, there is one $n$-dimensional vector space up to isomorphism. The arithmetical operations of addition and multiplication are upgraded to the algebraic operations of direct $\operatorname{sum}(\operatorname{dim}(V \oplus W)=\operatorname{dim} V+\operatorname{dim} W)$ and tensor product $(\operatorname{dim}(V \otimes W)=$ $(\operatorname{dim} V)(\operatorname{dim} W))$. The categorification has additional structure not found in the shadow. For example, the set $\mathbb{N}$ does not come equipped with maps between its elements, but any pair of vector spaces can be related by linear maps. Through the process of decategorification (in this example, taking the dimension of a vector space) we project the category back down to its shadow.

Suppose we also wish to categorify the operation of subtracting natural numbers. To accommodate negative numbers, we must expand our perspective to the category of chain complexes, and use Euler characteristic as our decategorifying operation. If $V, W$ are vector spaces with dimensions $n, m$ respectively, then the chain complex $0 \rightarrow V \rightarrow W \rightarrow 0$ (where $V$ and $W$ are assigned homological gradings 1 and 0$)$ has the property $\chi(0 \rightarrow V \rightarrow W \rightarrow 0)=\operatorname{dim} W-\operatorname{dim} V=m-n$.

Many well-known categorifications are algebraic upgrades of polynomials. As in the example above, the operation which recovers the polynomial is an Euler characteristic. To account for the various terms of the polynomial, we need to include an additional grading structure for our vector spaces. We will only discuss categorifications of single-variable polynomials, although this idea can be extended to multivariate polynomials as well.

Suppose we have a chain (or cochain) complex $\left(C^{i}, d^{i}\right)$, in which the elements of each chain group belong to a $\mathbb{Z}$-graded algebra with grading indexed by $j$. Each (co)chain group may be written as a direct sum $C^{i}=\oplus_{j} C^{i, j}$, and the complex has a graded Euler characteristic given by the formula

$$
\chi_{q}(C)=\sum_{i, j}(-1)^{i} q^{j} \operatorname{dim} C^{i, j}
$$


which is a polynomial in the variable $q$. If the $j$-grading is preserved by the differential, then the same formula holds for the homology of the complex $\left(C^{i}, d^{i}\right)$ :

$$
\chi_{q}(H)=\sum_{i, j}(-1)^{i} q^{j} \operatorname{dim} H^{i, j} .
$$

In low-dimensional topology, many polynomial invariants of knots and links have been categorified as the graded Euler characteristic of some homology theory. These include the Alexander polynomial [OS04], the Jones polynomial [Kho00], the $\mathfrak{s l}_{n}$ link polynomials [KR08a], and the HOMFLY-PT polynomial [KR08b]. In combinatorics, many polynomial invariants of graphs have received a similar treatment, including the chromatic polynomial [EH07, HGR05], the Tutte polynomial [JHR06], the dichromatic polynomial [Sto08], the Penrose polynomial [LR11], the magnitude polynomial [HW14], and the Stanley chromatic symmetric function [SY18].

\subsection{Knot theory}

Knots and links are one-dimensional models of physical strings and the ways in which they interact. We often imagine a link as a collection of circles in three-dimensional space, each homeomorphic to $S^{1}$, which may be knotted or intertwined with each other like pieces of string. To keep this picture close to physical reality, it is helpful to restrict the definition of a link to a set of piecewise linear curves.

Definition 1. [Lic97] A link $L$ of $m$ components is a subset of $S^{3}$ that consists of $m$ disjoint, piecewise linear, simple closed curves. A link of one component is a knot.

We define topological equivalence classes of links by the following relation. Two links $L_{1}$ and $L_{2}$ are said to be equivalent if there is a family of continuous maps $h_{t}: S^{3} \rightarrow S^{3}$ such that $h_{0}\left(L_{1}\right)=L_{1}$, $h_{1}\left(L_{1}\right)=L_{2}$, and $h_{t}\left(L_{1}\right)$ remains homeomorphic to $L_{1}$ for all $t \in[0,1]$. This continuous deformation is known as an ambient isotopy of $S^{3}$ from $L_{1}$ to $L_{2}$.

Rather than manipulating knots and links in three dimensions, it is often easier to project them down to the plane and look at their two-dimensional shadows. A projection of a link $L$ into $\mathbb{R}^{2}$, with under-strands and over-strands indicated at each crossing, is called a link diagram of $L$. Two diagrams represent isotopic links if and only if they are related by a sequence of diagrammatic operations known as Reidemeister moves (see Figure 2.1). 

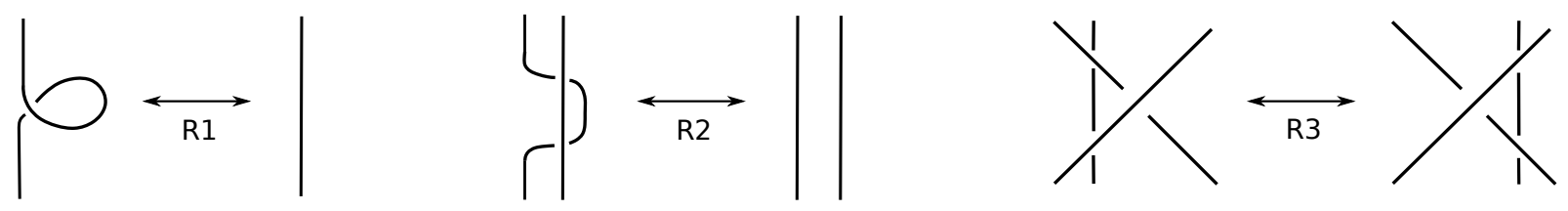

Figure 2.1: The Reidemeister moves.

Link invariants are mathematical objects, such as numbers, groups, or polynomials, which are computed from link diagrams and remain unchanged under the Reidemeister moves. Among the most well-known link invariants are the Alexander, Jones, and HOMFLY-PT polynomials. Each of these polynomials can be defined using a skein relation that measures the effect of local crossing changes in a diagram. We will define the Jones polynomial in detail before introducing Khovanov link homology.
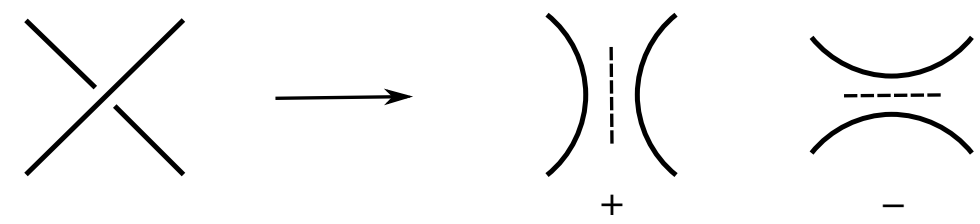

Figure 2.2: Positive and negative resolutions of a crossing.

Definition 2. The Kauffman bracket polynomial is a function that sends a link diagram $D$ in $\mathbb{R}^{2}$ to a Laurent polynomial $\langle D\rangle \in \mathbb{Z}\left[q, q^{-1}\right]$ defined by the following properties.

1. $\langle\bigcirc\rangle=q+q^{-1}$

2. $\langle D \sqcup \bigcirc\rangle=\left(q+q^{-1}\right)\langle D\rangle$

3. \langle\rangle$\rangle=\langle\bigvee\rangle-q\langle\rangle\langle\rangle$, where $\asymp$ and $\rangle\langle$ denote the link diagrams which are identical to $\lambda$ except for a positive or negative resolution at the given crossing (Figure 2.2).

The bracket polynomial defined above is only invariant under Reidemeister moves R2 and R3. To achieve invariance under R1, we multiply by a factor that depends on the crossings of the diagram. A normalizing factor $\left(q+q^{-1}\right)^{-1}$ may be applied so that the polynomial associated to the unknot is trivial.

Definition 3. The Jones polynomial of an oriented link $L$, denoted $J(L)$, is the Laurent polynomial in $\mathbb{Z}\left[q, q^{-1}\right]$ given by $J(L)=\frac{(-1)^{c_{-}} q^{c_{+}-2 c_{-}}\langle D\rangle}{q+q^{-1}}$ where $D$ is a diagram of $L$ with $c_{+}$positive crossings and $c_{-}$negative crossings according to the convention shown in Figure 2.3. We will refer to $\hat{J}(L)=(-1)^{c_{-}} q^{c_{+}-2 c_{-}}\langle D\rangle$ as the unnormalized Jones polynomial of $L$. 

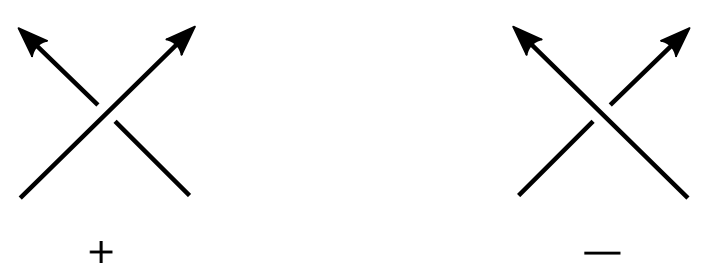

Figure 2.3: Convention for positive/negative crossings of an oriented link diagram.

\subsection{Khovanov homology}

Khovanov homology is an invariant of oriented links first introduced in [Kho00]. The Khovanov homology of $L$, denoted $K h(L)$, is a bigraded abelian group whose Euler characteristic is a version of the Jones polynomial $V(L)$. Since a link cobordism $L_{1} \rightarrow L_{2}$ induces a map $K h\left(L_{1}\right) \rightarrow K h\left(L_{2}\right)$, Khovanov homology is a functor from the category of links and link cobordisms to the category of abelian groups and group homomorphisms. It possesses a rich algebraic structure that includes new invariants (such as the Rasmussen $s$-invariant [Ras10]) and homological operations such as spectral sequences [PS16]. It distinguishes many links which the Jones polynomial does not. In addition, $K h(L)$ is an unknot detector [KM11], a property conjectured but not yet proven in the case of the Jones polynomial. Since its discovery, Khovanov homology has been applied to link topology [BS15, Ng05], Heegaard Floer homology for 3-manifolds [Bal11, OS05], and gauge theory [Wit12].

\subsubsection{Construction}

In this section, we review the construction of Khovanov homology following [BN02] and [Vir04].

Definition 4. Let $M=\bigoplus_{j} M^{j}$ be a graded vector space where the $M^{j}$ are the homogeneous components of $M$. The graded dimension of $M$ is defined to be $q \operatorname{dim} M=\sum_{j} q^{j} \operatorname{dim} M^{j}$. Let $\cdot\{\ell\}$ be the degree shift operation on a graded vector space $M$ given by $M\{\ell\}^{j}=M^{j-\ell}$.

Definition 5. Let $\left\{C^{i}\right\}$ be a chain complex. The height shift operation on this complex, denoted $\cdot[s]$, adjusts the grading such that $C[s]^{i}=C^{i-s}$.

Let $D$ be a diagram of link $L$. The construction of Khovanov homology builds on so-called Kauffman states described in Definition 6 where each crossing in a diagram $D$ of link $L$ is assigned a choice of a positive or negative resolution, also known as a "smoothing" of the crossing (see Figure $2.2)$.

Definition 6. A Kauffman state of $D$ is a collection of disjoint circles, denoted $D_{s}$, obtained by resolving each crossing of $D$ in either the positive or negative way according to a function 
$s:\{$ crossings of $D\} \rightarrow\{-1,1\}$. An enhanced Kauffman state $S$ is a Kauffman state $s$ in which each circle in $D_{s}$ is assigned a label 1 or $x$. Let $n_{+}(s)$ denote the number of positive smoothings in Kauffman state $s$, and $n_{-}(s)$ denote the number of negative smoothings.

Let $\mathcal{A}_{2}=\mathbb{Z}[x] /\left(x^{2}\right)$ be the graded $\mathbb{Z}$-module whose generators 1 and $x$ have degree 1 and -1 , respectively. The graded dimension of a $\mathbb{Z}$-module or graded vector space $M$ is defined as qdim $M=\sum_{j} q^{j} \operatorname{dim} M^{j}$. We use $\mathcal{A}_{2}$ in the construction of Khovanov homology because its graded $\operatorname{dimension} q \operatorname{dim} \mathcal{A}_{2}=q+q^{-1}$ is the bracket polynomial of the unknot.

Fix an order on the $n$ crossings of $D$, and let each Kauffman state be represented by a tuple in $\{0,1\}^{n}$ with 0 s for positive smoothings and 1 s for negative smoothings. The $2^{n}$ Kauffman states of $D$ are in one-to-one correspondence with the vertices of an $n$-dimensional cube: state $s$ corresponds to vertex $\alpha=\left(\alpha_{1}, \alpha_{2}, \ldots, \alpha_{n}\right)$ where $\alpha_{k}=0$ if the $k$ th crossing is resolved with a positive smoothing in $s$, and $\alpha_{k}=1$ if it is resolved with a negative smoothing. To the vertex $\alpha$, we assign the graded $\mathbb{Z}$-module $C_{\alpha}(D)=\mathcal{A}_{2}^{\otimes k(s)}$, where $k(s)$ is the number of circles in $s$.

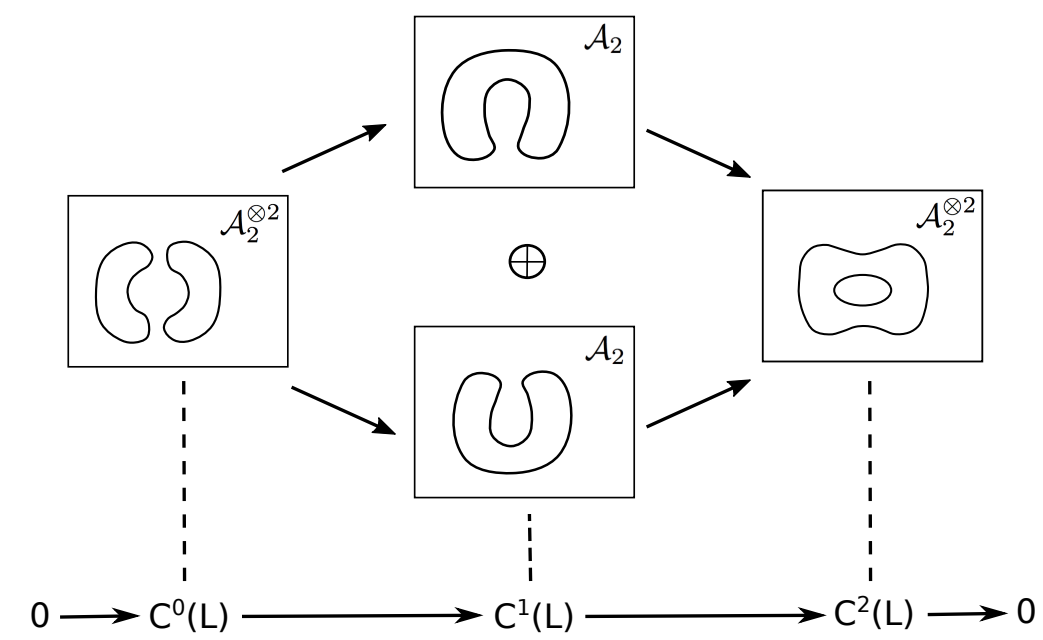

Figure 2.4: Kauffman states and Khovanov chain groups for the Hopf link $L=2_{1}$.

The cochain groups in the Khovanov complex are obtained as direct sums along the diagonals of the cube:

$$
C^{i}(D)=\bigoplus_{|\alpha|=i} C_{\alpha}(D)
$$

where $|\alpha|$ represents the number of 1 s in the label of vertex $\alpha$. We can think of $C^{i}(D)$ as a group freely generated by enhanced states of $D$ with $i$ negative smoothings. Let $C^{i, j}(D)$ denote the subgroup of $C^{i}(D)$ generated by elements whose $\mathbb{Z}$-module grading is $j$.

To define a differential on this cochain complex, we first define maps along the edges of the cube of resolutions. Suppose Kauffman states $s$ and $s^{\prime}$ only differ at the $k$ th crossing, where $s$ has 
the positive smoothing and $s^{\prime}$ has the negative smoothing. The corresponding vertices of the cube $\alpha$ and $\alpha^{\prime}$ differ only in the $k$ th coordinate, where $\alpha_{k}=0$ and $\alpha_{k}^{\prime}=1$. Thus there is an edge of the cube from $\alpha$ to $\alpha^{\prime}$, which we denote $e$. We define the map $d_{e}: C_{\alpha}(D) \rightarrow C_{\alpha^{\prime}}(D)$ as follows. If $s^{\prime}$ is obtained from $s$ by joining two circles, $d_{e}$ is the map $m: \mathcal{A}_{2} \otimes \mathcal{A}_{2} \rightarrow \mathcal{A}_{2}$ that multiplies the labels on those circles. If $s^{\prime}$ is obtained from $s$ by splitting one circle into two, $d_{e}$ is the comultiplication map $\Delta: \mathcal{A}_{2} \rightarrow \mathcal{A}_{2} \otimes \mathcal{A}_{2}$ that sends $1 \mapsto 1 \otimes x+x \otimes 1$ and $x \mapsto x \otimes x$. The differential $d^{i}: C^{i}(D) \rightarrow C^{i+1}(D)$ is defined to be

$$
d^{i}=\sum_{\left\{d_{e}:|\alpha|=i\right\}}(-1)^{\xi} d_{e}
$$

where $C_{\alpha}(D)$ is the domain of $d_{e}$ and the sign $(-1)^{\xi}$ is chosen as follows.

Suppose the $k$ th coordinate of $\alpha$ is being changed from 0 to 1 along edge $e$ from $\alpha$ to $\alpha^{\prime}$. Let $\xi \in\{0,1, *\}^{n}$ be the $n$-tuple which has a $*$ in the $k$ th coordinate and agrees with $\alpha$ and $\alpha^{\prime}$ in every other coordinate. If the number of 1 s preceding the $*$ is odd, then $(-1)^{\xi}=-1$; if the number of 1 s is even, then $(-1)^{\xi}=1$. This assignment ensures that every square face of the cube has a single edge whose associated map has opposite sign from the maps on the other three edges of the square. Since $m$ and $\Delta$ are (co)associative and (co)commutative respectively, each square face anti-commutes, and so $d^{2}=0$.

The chain complex $\mathcal{C}(D)=\left(C^{i}(D), d^{i}\right)$ is the Khovanov (co)chain complex of $D$. Since the differential preserves degree, $\mathcal{C}(D)$ is a bigraded chain complex. In accordance with the grading conventions found in [BN02], we shift the original complex by a factor that depends on the number of positive and negative crossings in $D$ (denoted $c_{+}$and $c_{-}$, respectively). The shifted complex is denoted by $\overline{\mathcal{C}}(D)=\mathcal{C}(D)\left[-c_{-}\right]\left\{c_{+}-2 c_{-}\right\}$where $\cdot\{\ell\}$ and $\cdot[s]$ are the degree and height shift operations described above.

The homology of $\overline{\mathcal{C}}(D)$ is denoted $K h(D)$, the Khovanov homology of diagram $D$. (Technically, this is a cohomology theory because the differential increases the homological degree, but we will follow general usage in calling it homology.) Khovanov homology is a link invariant ([Kho00], [BN02]) with graded Euler characteristic $\chi_{q}(K h(L))=\sum_{i}(-1)^{i}$ qdim $\left(K h^{i}(L)\right)=\hat{J}(L)$ where $\hat{J}(L)$ is the unnormalized Jones polynomial. This polynomial can also be expressed [Kau11] as a state sum formula

$$
\hat{J}(L)=(-1)^{c_{-}} q^{c_{+}-2 c_{-}} \sum_{i=0}^{c_{+}+c_{-}}(-1)^{i} \sum_{\left\{S: n_{-}(S)=i\right\}} q^{i}\left(q+q^{-1}\right)^{|S|}
$$

where $S$ is an enhanced Kauffman state with $|S|$ connected components.

In Khovanov homology, the Jones polynomial skein relation is categorified as a long exact 
sequence of homology groups:

$$
\left.\left.\ldots \rightarrow K h^{i-1}(\asymp) \rightarrow K h^{i}(〈) \rightarrow K h^{i} \searrow\right) \rightarrow K h^{i} \asymp\right) \rightarrow \ldots
$$

\subsubsection{Supported gradings and diagonals}

If $L$ has an odd number of components, then the quantum grading $K h^{*, m}(L)=0$ for even $m$; if the number of components is even, $K h^{*, m}(L)=0$ for odd $m$. These zero rows are typically omitted from tabular diagrams of Khovanov homology. To give a more detailed picture of $K h(L)$, we can describe its extent in the $j$-dimension, as well as the number of slope 2 diagonals on which it lives.

Definition 7. Let $j_{\min }$ be the minimal quantum grading on which $K h(L)$ is nontrivial, and let $j_{\max }$ be the highest. Then we define the quantum span of homology $K h(L)$ as: $q \operatorname{span}(K h(L))=$ $j_{\max }-j_{\min }+1$.

A b-diagonal in Khovanov homology is a set of bigradings $(i, j)$ for which $-2 i+j=b$. If $K h(L)$ is non-trivial on the set of diagonals $\left\{-2 i+j=a_{i}\right\}$, then we define homological width of $K h(L)$ to be $\mathrm{hw}(K h(L))=a_{\max }-a_{\min }+1$ where $a_{\max }, a_{\min }$ are the maximum and minimum values of $a_{i}$. Torsion width of homology is defined analogously, and denoted $\mathrm{hw}^{t}(K h(L))$.

The span of the Jones polynomial is the difference between the highest and lowest degrees of its terms. While the quantum span of Khovanov homology cannot be less than the span of the Jones polynomial, it may be larger due to extreme bigradings whose contributions to the Euler characteristic cancel each other out. For an example, see $K h\left(10_{132}\right)$ in [Kat].

The signature of a link, denoted $\sigma(L)$, is the signature of the Seifert matrix obtained from any diagram of $L$. For many links, $K h(L)$ lies only on the $(\sigma(L) \pm 1)$-diagonals. [Kho03]. When $K h(L)$ lies only on these two diagonals, we say it is thin; otherwise, we say it is thick.

Theorem 1. [Lee05] If $L$ is an alternating link, then $K h(L)$ is thin.

Manolescu and Oszvath later defined a more general class of links, known as quasi-alternating, for which Khovanov homology is thin. However, there exist non-quasi-alternating links for which $K h(L)$ is thin (see e.g. [Sta12]) and no general criterion for thinness is known.

When $K h(L)$ is thin, all ranks on the $(\sigma(L)+1)$-diagonal match those on the $(\sigma(L)-1)$-diagonal up to a "knight move" shift of degree $(1,4)$, except for a finite number of "exceptional pairs" with the form $\mathbb{Z} \oplus \mathbb{Z}\{2\}$. In [Ras10], the location of one exceptional pair is used to define the Rasmussen $s$-invariant, which gives a lower bound for the slice genus of a knot.

Theorem 2. [Lee05, Shu16] If $L$ is a link such that $\mathrm{hw}(K h(L))=2$, then $K h(L)$ consists of a finite number of summands of the form $\mathbb{Z} \oplus \mathbb{Z}[1]\{4\} \oplus \mathbb{Z}_{2}[1]\{2\}$ together with a finite number of summands of the form $\mathbb{Z} \oplus \mathbb{Z}\{2\}$. 


\subsubsection{Torsion}

Khovanov homology frequently contains $\mathbb{Z}_{2}$ torsion, while torsion of other orders is relatively rare. Based on computations up to 13 crossings, Shumakovitch [Shu14] conjectured that $\mathbb{Z}_{2}$ torsion is present in the Khovanov homology of all knots and links, except for disjoint unions and connected sums of unknots and Hopf links. If true, this statement implies that Khovanov homology detects the unknot, a result whose only known proof requires techniques from gauge theory [KM11]. In recent work, Shumakovitch demonstrates that if $K h(L)$ is thin, then it contains only $\mathbb{Z}_{2}$ torsion [Shu16].

The smallest known knot with odd torsion is the torus knot $T(5,6)$, which has $\mathbb{Z}_{3}$ and $\mathbb{Z}_{5}$ torsion in its Khovanov homology [Kat, MPS $\left.{ }^{+} 17\right]$. Through twist deformations of torus links, $\left[\mathrm{MPS}^{+} 17\right]$ found infinite families of links which have torsion of order $n$ for $2<n<9$, as well as infinite families with torsion of order $2^{s}$ for $s<24$.

Reduced Khovanov homology, denoted $\widetilde{K h}(L)$, generally has fewer torsion groups than the unreduced version. For example, $\widetilde{K h}(L)$ is torsion-free if $L$ is alternating [Shu14]. However, odd torsion is also possible; for example, in $T(7,8)$ and $T(7,9)$ [MPS $\left.{ }^{+} 17\right]$. Odd Khovanov homology $K h_{\text {odd }}(L)$ [ORS13], which agrees with $K h(L)$ over $\mathbb{Z}_{2}$ coefficients, has odd torsion for knots with crossing numbers as low as 8 . Unlike the standard (even) version of reduced homology, $\widehat{K h_{\text {odd }}}(L)$ has the same orders of torsion as $K h_{\text {odd }}(L)$ for any given link [Shu11].

\subsection{Chromatic graph homology}

Inspired by Khovanov's categorification of the Jones polynomial, Helme-Guizon and Rong constructed a bigraded chain complex for graphs whose Euler characteristic is the chromatic polynomial [HGR05]. We review the definition of this polynomial along with several important properties.

Let $G$ be a finite, undirected graph with vertex set $V(G)$ and edge set $E(G)$. We will often denote the cardinalities of these sets by $v=|V(G)|$ and $E=|E(G)|$. If $G$ has an edge between vertices $x, y \in V(G)$, we write the corresponding element in $E(G)$ as $\{x, y\}$.

Definition 8. Define the set of $\lambda$-colors to be the set of integers $\{1, \ldots, \lambda\} . A \lambda$-coloring of $G$ is an assignment of $\lambda$-colors to the elements of $V(G)$ such that if $x, y \in V(G)$ and $\{x, y\} \in E(G)$, then the colors assigned to $x$ and $y$ are distinct.

Definition 9. The chromatic polynomial of graph $G$, denoted $P_{G}(\lambda)$, counts the number of $\lambda$ colorings of $G$.

Proposition 1. The chromatic polynomial is the unique graph polynomial defined by the following properties:

1. If $\bar{K}_{n}$ is the empty graph with $n$ vertices and no edges, then $P_{\bar{K}_{n}}(\lambda)=\lambda^{n}$. 
2. If $e \in E(G)$, then the following deletion-contraction relation holds: $P_{G}(\lambda)=P_{G-e}(\lambda)-$ $P_{G / e}(\lambda)$.

Note that if $G$ has a loop at vertex $v$, then there are no possible colorings and $P_{G}(\lambda)=0$ for all $\lambda$. If $G^{\prime}$ is the graph $G$ with multiple edges added between vertices $v, w \in V(G)$, then $P_{G}(\lambda)=P_{G^{\prime}}(\lambda)$ for all $\lambda$.

A spanning subgraph of $G$ is a subgraph which contains all the vertices of $G$. The chromatic polynomial admits an inclusion-exclusion type formula

$$
P_{G}(\lambda)=\sum_{i=0}^{E}(-1)^{i} \sum_{\{s:|s|=i\}} \lambda^{k(s)}
$$

where $s$ is a spanning subgraph with $|s|$ edges and $k(s)$ connected components [HGR05]. This formula plays a role in the construction of chromatic homology analogous to that played by the Jones polynomial state sum formula Eq.(2.2) in Khovanov homology. The spanning subgraphs are used in a manner similar to Kauffman states of a link diagram.

\subsubsection{Construction}

The construction of chromatic homology depends on the choice of an algebra $\mathcal{A}$. In [HGR05], this was originally envisioned as $\mathcal{A}_{2}=\mathbb{Z}[x] /\left(x^{2}\right)$, the polynomial algebra which appears in the construction of Khovanov homology. The chromatic chain complex over $\mathcal{A}_{2}$ is isomorphic to the Khovanov chain complex up until the first appearance of comultiplication in the latter. In general, $\mathcal{A}$ may be any associative, $\mathbb{Z}$-graded algebra with unit [HGR12].

We label the connected components of spanning subgraphs with generators of $\mathcal{A}$ to obtain enhanced state graphs, analogous to enhanced Kauffman states. If we substitute $\lambda=$ qdim $\mathcal{A}$ in the state sum formula $(2.3)$, then $P_{G}(\operatorname{qdim} \mathcal{A})$ can be realized as the Euler characteristic of the homology theory that follows.

Fix an ordering on the edge set $E(G)=\left\{e_{1}, e_{2}, \ldots, e_{n}\right\}$. As with the Khovanov cube of resolutions, there are $2^{n}$ possible spanning subgraphs of $G$ that can be arranged as vertices of an $n$-dimensional cube. Each vertex has a label $\left(\alpha_{1}, \alpha_{2}, \ldots, \alpha_{n}\right) \in\{0,1\}^{n}$, where $\alpha_{k}=1$ if and only if the $k$ th edge is present in the corresponding spanning subgraph $s$. To each vertex $\alpha$, we assign the graded $\mathbb{Z}$-module $C_{\mathcal{A}, \alpha}(G)=\mathcal{A}^{\otimes k(s)}$, where $k(s)$ is the number of connected components in $s$; see Figure 2.5. Let $C_{\mathcal{A}}^{i}(G)$ be the group freely generated by enhanced state graphs of $G$ with $i$ edges, and let $C_{\mathcal{A}}^{i, j}(G)$ be the subgroup generated by elements of $C_{\mathcal{A}}^{i}(G)$ whose $\mathbb{Z}$-module grading is $j$.

Each edge of the cube corresponds to a map $d_{e}$, defined as follows. Suppose spanning subgraphs $s$ and $s^{\prime}$ are identical except that $s^{\prime}$ contains the $k$ th edge and $s$ does not. The corresponding vertices of the cube $\alpha$ and $\alpha^{\prime}$ differ only in the $k$ th coordinate, where $\alpha_{k}=0$ and $\alpha_{k}^{\prime}=1$. Thus there is 
an edge of the cube from $\alpha$ to $\alpha^{\prime}$. If the $k$ th edge (here denoted $e$ ) joins different components of $s$, then $d_{e}: C_{\mathcal{A}, \alpha}(G) \rightarrow C_{\mathcal{A}, \alpha^{\prime}}(G)$ is the map $m: \mathcal{A} \otimes \mathcal{A} \rightarrow \mathcal{A}$ that multiplies the labels on these components. If the addition of edge $e$ preserves the number of connected components in $s$, then $d_{e}$ is the identity map on $\mathcal{A}$.

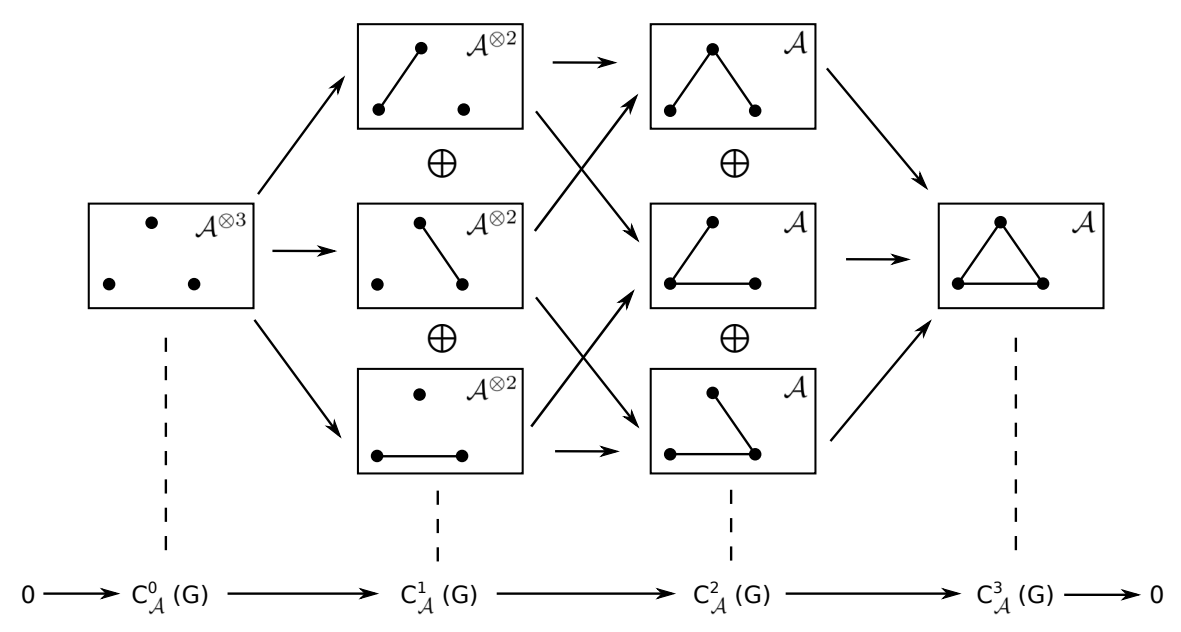

Figure 2.5: Subgraphs and chromatic chain groups of a 3 -cycle $G=P_{3}$.

The chromatic differential $d^{i}: C_{\mathcal{A}}^{i}(G) \rightarrow C_{\mathcal{A}}^{i+1}(G)$ is defined by

$$
d^{i}=\sum_{\left\{d_{e}:|\alpha|=i\right\}}(-1)^{\xi} d_{e}
$$

where the sign $(-1)^{\xi}$ is chosen in analogous fashion to the sign in the Khovanov differential. The chain complex $C_{\mathcal{A}}(G)=\left(C_{\mathcal{A}}^{i}(G), d^{i}\right)$ is the chromatic chain complex of $G$. The homology of $C_{\mathcal{A}}(G)$ is denoted $H_{\mathcal{A}}(G)$ and called the chromatic homology of graph $G$.

The graded Euler characteristic of $H_{\mathcal{A}}(G)$ is $\chi_{q}\left(H_{\mathcal{A}}(G)\right)=\sum_{i}(-1)^{i} \mathrm{qdim}\left(H_{\mathcal{A}}^{i}(G)\right)$, and since $d^{i}$ is a degree preserving differential, it recovers the evaluation of the chromatic polynomial at $\lambda=\operatorname{qdim} \mathcal{A}$ :

$$
\chi_{q}\left(H_{\mathcal{A}}(G)\right)=\chi_{q}\left(C_{\mathcal{A}}(G)\right)=\sum_{i=0}^{E}(-1)^{i} \sum_{\{s:|s|=i\}}(\operatorname{qdim} \mathcal{A})^{k(s)}=P_{G}(\operatorname{qdim} \mathcal{A})
$$

With the special choice of algebra $\mathcal{A}=\mathbb{Z}[x] /\left(x^{2}\right)=\mathcal{A}_{2}$, this Euler characteristic is $P_{G}\left(\right.$ qdim $\left.\mathcal{A}_{2}\right)=$ $P_{G}(q+1)$.

Analogous to the categorification of the Jones polynomial skein relation, the deletion-contraction 
formula for the chromatic polynomial

$$
P_{G}(\lambda)=P_{G-e}(\lambda)-P_{G / e}(\lambda)
$$

is categorified by to the short exact sequence [HGR05] of chain groups

$$
0 \rightarrow C_{\mathcal{A}}^{i-1, j}(G / e) \rightarrow C_{\mathcal{A}}^{i, j}(G) \rightarrow C_{\mathcal{A}}^{i, j}(G-e) \rightarrow 0
$$

which induces a long exact sequence in chromatic homology:

$$
0 \rightarrow H_{\mathcal{A}}^{0, j}(G) \rightarrow H_{\mathcal{A}}^{0, j}(G-e) \rightarrow H_{\mathcal{A}}^{0, j}(G / e) \rightarrow \ldots \rightarrow H_{\mathcal{A}}^{i-1, j}(G / e) \rightarrow H_{\mathcal{A}}^{i, j}(G) \rightarrow H_{\mathcal{A}}^{i, j}(G-e) \rightarrow \ldots
$$

Both $P_{G}(\lambda)$ and $H_{\mathcal{A}}(G)$ are trivial if $G$ has a loop, and both remain unchanged if multiple edges are added between two vertices. Therefore, unless otherwise stated, we will assume that $G$ is a simple graph with no loops or multiple edges. For simplicity, we assume $G$ is connected, since the following formula gives the chromatic homology of any graph in terms of the chromatic homology of its connected components.

Theorem 3. [HGR12, Theorem 3.6] Let $G_{1}, G_{2}$ be graphs with disjoint union $G_{1} \sqcup G_{2}$. Then

$$
H_{\mathcal{A}}^{i}\left(G_{1} \sqcup G_{2}\right) \cong\left[\bigoplus_{p+q=i} H_{\mathcal{A}}^{p}\left(G_{1}\right) \otimes H_{\mathcal{A}}^{q}\left(G_{2}\right)\right] \oplus\left[\bigoplus_{p+q=i+1} H_{\mathcal{A}}^{p}\left(G_{1}\right) * H_{\mathcal{A}}^{q}\left(G_{2}\right)\right]
$$

where $*$ denotes the torsion product of two abelian groups.

Corollary 1. [HGR12, Example 4.1] The chromatic homology of the empty graph is given by

$$
H_{\mathcal{A}}^{i}\left(\bar{K}_{n}\right)= \begin{cases}\mathcal{A}^{\otimes n} & i=0 \\ 0 & \text { otherwise. }\end{cases}
$$

A pendant edge of a graph is an edge which is attached to a vertex of degree one. Adding a pendant edge to a graph $G$ multiplies its chromatic polynomial $P_{G}(\lambda)$ by a factor of $(\lambda-1)$. This property is categorified in chromatic homology. For a given algebra $\mathcal{A}$ with unit $1_{\mathcal{A}}$, we can write $\mathcal{A}=\mathbb{Z}_{1_{\mathcal{A}}} \oplus \mathcal{A}^{\prime}$

Theorem 4. [HGR12, Prop. 3.4] If e is a pendant edge of $G$, then $H_{\mathcal{A}}(G)=H_{\mathcal{A}}(G / e) \otimes \mathcal{A}^{\prime}$. 
Corollary 2. [HGR12, Example 4.3] Let $T_{n}$ be the tree with $n+1$ vertices and $n$ edges. Then

$$
H_{\mathcal{A}}^{i}\left(T_{n}\right)= \begin{cases}\mathcal{A} \otimes \mathcal{A}^{\prime \otimes n} & i=0 \\ 0 & \text { otherwise }\end{cases}
$$

\subsubsection{Chromatic homology over $\mathbb{Z}[x] /\left(x^{2}\right)$}

Although the construction may be more general, we focus on chromatic homology over polynomial algebras of the form $\mathcal{A}_{m}=\mathbb{Z}[x] /\left(x^{m}\right)$, following [HGPR06] and [PPS09]. Of particular interest is $\mathcal{A}_{2}=\mathbb{Z}[x] /\left(x^{2}\right)$, since this is the same algebra used to construct Khovanov homology.

Definition 10. Let $i_{\text {min }}$ be the minimal homological grading on which $H_{\mathcal{A}}(G)$ is nontrivial, and let $i_{\text {max }}$ be the highest. Then we define the homological span of $H_{\mathcal{A}}(G)$ as: $\operatorname{hspan}\left(H_{\mathcal{A}}(G)\right)=i_{\max }-$ $i_{\text {min }}+1$. The quantum span of $H_{\mathcal{A}}(G)$ is defined similarly: $q \operatorname{span}\left(H_{\mathcal{A}}(G)\right)=j_{\text {max }}-j_{\text {min }}+1$.

$A$ b-diagonal in chromatic homology is a set of bigradings $(i, j)$ for which $i+j=b$. If $H_{\mathcal{A}}(G)$ is non-trivial on the set of diagonals $\left\{i+j=a_{i}\right\}$, then we define homological width of $H_{\mathcal{A}}(G)$ to be $\mathrm{hw}\left(H_{\mathcal{A}}(G)\right)=a_{\max }-a_{\text {min }}+1$ where $a_{\max }, a_{\min }$ are the maximum and minimum values of $a_{i}$. Torsion width of homology is defined analogously, and denoted $\mathrm{hw}^{t}\left(H_{\mathcal{A}}(G)\right)$.

Proposition 2. [HGPR06, Cor. 16] $H_{\mathcal{A}_{2}}(G)$ is concentrated on the $v$-and $(v-1)$-diagonals, where $v$ is the number of vertices in $G$. Torsion exists only on the $v$-diagonal.

Chromatic homology of a connected graph over $\mathcal{A}_{2}$ closely resembles the Khovanov homology of an alternating link, occupying exactly two diagonals and having only $\mathbb{Z}_{2}$ torsion [LS17]. Chmutov, Chmutov and Rong [CCR08] proved that the ranks of $H_{\mathcal{A}_{2}}(G ; \mathbb{Q})$ on these two diagonals are related by a knight move isomorphism with bidegree $(1,-4)$. Lowrance and Sazdanovic [LS17] showed that over integral coefficients, torsion is always found between $\mathbb{Z} \mathrm{s}$ in a knight move pair, and so $H_{\mathcal{A}_{2}}(G)$ can be constructed using only information about ranks.

Theorem 5. [LS17, Theorem 1.4] $H_{\mathcal{A}_{2}}(G ; \mathbb{Z})$ is determined by the chromatic polynomial of $G$. Specifically, $H_{\mathcal{A}_{2}}(G ; \mathbb{Z})$ consists of a finite number of summands of the form $(\mathbb{Z} \oplus \mathbb{Z}[1]\{-2\} \oplus$ $\left.\mathbb{Z}_{2}[1]\{-1\}\right)[i]\{v-i\}$ with $i \geq 0$, plus a summand $\mathbb{Z}\{v\} \oplus \mathbb{Z}\{v-1\}$ in homological grading $i=0$ if $G$ is bipartite.

\subsubsection{Chromatic homology over $\mathbb{Z}[x] /\left(x^{m}\right)$ for $m>2$}

For values of $m$ greater than $2, H_{\mathcal{A}_{m}}(G)$ may lie on many diagonals, and all but one of these are allowed to contain torsion.

Proposition 3. [HGPR06, Cor. 13] Let $G$ be a connected graph with v vertices. 


$$
H_{\mathcal{A}_{m}}^{i, j}(G) \neq 0 \Rightarrow\left\{\begin{array}{l}
0 \leq i \leq v-2 \\
i+j \geq v-1 \\
(m-1) i+j \leq(m-1) v
\end{array} \quad \text { tor } H_{\mathcal{A}_{m}}^{i, j}(G) \neq 0 \Rightarrow\left\{\begin{array}{l}
1 \leq i \leq v-2 \\
i+j \geq v \\
(m-1) i+j \leq(m-1) v
\end{array}\right.\right.
$$

Computations suggest that $\mathbb{Z}_{m}$ torsion is most common in $H_{\mathcal{A}_{m}}(G)$, and that $\mathbb{Z}_{2}$ appears in the homology of wheel graphs and complete graphs. In general, however, there are no restrictions on the orders of torsion that may appear. In [PPS09], the authors construct a cell complex which is a partial geometric realization of the bigrading $H_{\mathcal{A}_{3}}^{1,2 v-3}(G)$ and show that this complex may be used to produce torsion of any kind.

Proposition 4. [PPS09, Cor. 5.2] For any finite abelian group $T$, there is a simple graph $G$ such that tor $H_{\mathcal{A}_{3}}^{1,2 v-3}(G)=T$.

\subsection{Correspondence between Khovanov and chromatic homologies}

Given a diagram $D$ of a link $L$, let $s_{+}(D)$ be the Kauffman state of $D$ which has a positive smoothing at each crossing. The all-positive state graph $G_{+}(D)$ consists of one vertex for each circle in $s_{+}(D)$, with an edge connecting any pair of circles related by a crossing in $D$ (see Figure 2.6). (For alternating links, $G_{+}(D)$ is often referred to as the Tait graph of $D$.) This construction may also be applied to any other Kauffman state of $G$. We denote by $s_{-}(D)$ the Kauffman state that results from applying the negative smoothing at each crossing, with $G_{-}(D)$ the all-negative state graph.

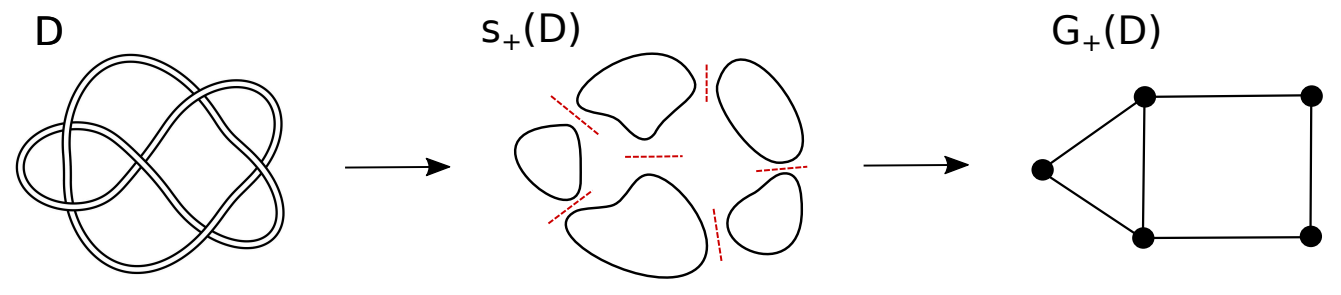

Figure 2.6: Diagram of $5_{1}$ and its corresponding planar graph.

The connection between Khovanov homology and state graphs of a diagram was first realized by Przytycki via Hochschild homology, a homology theory for associative algebras introduced in [Hoc45]. Our definition of Hochschild homology follows [Lod98, Prz10]. Let $\mathbb{k}$ be a commutative ring and let $\mathcal{A}$ a $\mathbb{k}$-algebra with unit. Let $\mathbb{M}$ be a bimodule over $\mathcal{A}$; that is, a module on which $\mathcal{A}$ operates linearly such that $(a m) a^{\prime}=a\left(m a^{\prime}\right)$ for all $a, a^{\prime} \in \mathcal{A}, m \in \mathbb{M}$. In this section, let $\mathcal{A} \otimes \mathcal{B}$ denote the tensor product of two algebras over the ring $\mathbb{k}$.

Definition 11. The Hochschild chain complex $C_{*}(\mathcal{A}, \mathbb{M})$ of the algebra $\mathcal{A}$ with coefficients in $\mathbb{M}$ is 
defined as

$$
\ldots \rightarrow \mathbb{M} \otimes \mathcal{A}^{\otimes n} \rightarrow \mathbb{M} \otimes \mathcal{A}^{\otimes n-1} \rightarrow \ldots \rightarrow \mathbb{M} \otimes \mathcal{A} \rightarrow \mathbb{M}
$$

where $C_{n}(\mathcal{A}, \mathbb{M})=\mathbb{M} \otimes \mathcal{A}^{\otimes n}$ and the Hochschild boundary is the $\mathbb{k}$-linear map $b: \mathbb{M} \otimes \mathcal{A}^{\otimes n} \rightarrow$ $\mathbb{M} \otimes \mathcal{A}^{\otimes n-1}$ given by $b=\sum_{i=0}^{n}(-1)^{i} d_{i}$, with face maps $d_{i}$ defined as follows:

$$
\begin{aligned}
& d_{0}\left(m, a_{1}, \ldots, a_{n}\right)=\left(m a_{1}, a_{2}, \ldots, a_{n}\right) \\
& d_{i}\left(m, a_{1}, \ldots, a_{n}\right)=\left(m, a_{1}, \ldots, a_{i} a_{i+1}, \ldots, a_{n}\right) \text { for } 1 \leq i \leq n-1 \\
& d_{n}\left(m, a_{1}, \ldots, a_{n}\right)=\left(a_{n} m, a_{1}, \ldots, a_{n-1}\right)
\end{aligned}
$$

The nth homology group of this chain complex is called the nth Hochschild homology group of $\mathcal{A}$ with coefficients in $\mathbb{M}$ and denoted $H_{n}(\mathcal{A}, \mathbb{M})$. In the case $\mathbb{M}=\mathcal{A}$, we use the notation $H H_{n}(\mathcal{A})$ for this homology.

The Khovanov homology of the $(2, n)$ torus link is isomorphic to part of the Hochschild homology of the algebra $\mathcal{A}_{2}$. The standard diagram of this link has an all-positive state graph which is a cycle (or polygon) $P_{n}$ (Figure 2.7), and the chromatic homology $H_{\mathcal{A}_{2}}\left(P_{n}\right)$ is also related to the Hochschild homology of $\mathcal{A}_{2}$. The following result explains the correspondence precisely for any $\mathcal{A}_{m}$.

$T(2,7)$

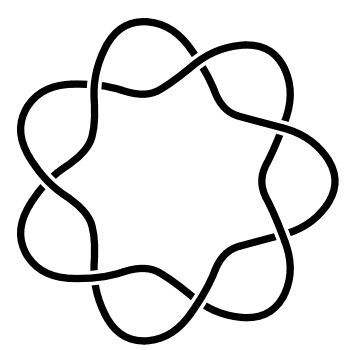

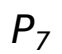

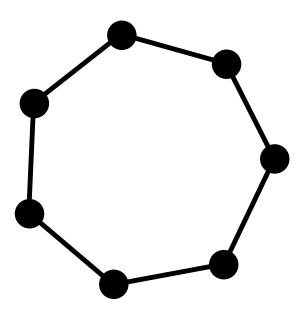

Figure 2.7: Diagram of torus knot $T(2,7)$ and its corresponding all-positive state graph.

Theorem 6. [Prz10] Let $H H\left(\mathcal{A}_{m}\right)$ be the Hochschild homology of $\mathcal{A}_{m}$. For $i>0$, Hochschild homology determines the chromatic homology of a cycle graph $P_{n}$ as follows:

$$
H H_{i-n-1, j}\left(\mathcal{A}_{m}\right) \cong H_{\mathcal{A}_{m}}^{i, j}\left(P_{n}\right) \cong \begin{cases}\mathbb{Z}_{m} & \text { if } i<n-1, n-i \text { even, } j=\frac{n-i}{2} m \\ \mathbb{Z} & \text { if } i<n-1,\left\lfloor\frac{n-i-1}{2}\right\rfloor m+1 \leq j \leq\left\lfloor\frac{n-i-1}{2}\right\rfloor m+m-1 \\ 0 & \text { otherwise }\end{cases}
$$

Chromatic homology may be considered as a generalization of Hochschild homology of $\mathcal{A}_{m}$ to 
graphs with multiple cycles.

Definition 12. The girth of a graph $G$, denoted $\ell(G)$, is the length of the shortest cycle in $G$. We adopt the convention that the girth of a tree is zero, as opposed to considering the girth of a tree to be infinite (see [Bol98, Die00]).

The chromatic chain complex $C_{\mathcal{A}}^{i}\left(G_{+}(D)\right)$ is isomorphic to the Khovanov chain complex of $L$ in homological gradings $i=0$ through $i=\ell-1$. For these gradings, the enhanced Kauffman states of $D$ and the enhanced state graphs of $G_{+}(D)$ have the same number of components. The edge maps $d_{e}$ on each cube complex multiply the labels associated to these components. In $C_{\mathcal{A}}^{\ell}\left(G_{+}(D)\right)$, one of the spanning subgraphs with $\ell$ edges contains a cycle. The closure of a cycle in the $\ell$ th grading indicates that one of the edge maps in Eq. (2.1) is a comultiplication in the Khovanov cube complex. It follows that the chromatic homology in degree $\ell$ and all subsequent gradings may differ from the corresponding gradings in Khovanov homology.

The following result describes the partial correspondence between the Khovanov homology of $L$ and the chromatic homology of the corresponding graph $G_{+}(D)$.

Theorem 7. [Prz10, PS14] Let D be a diagram of link $L$ with $c_{-}$negative crossings and $c_{+}$positive crossings. Suppose $G_{+}(D)$ has $v$ vertices and positive girth $\ell$. Let $p=i-c_{-}$and $q=v-2 j+c_{+}-2 c_{-}$. For $0 \leq i<\ell$ and $j \in \mathbb{Z}$, there is an isomorphism $H_{\mathcal{A}_{2}}^{i, j}\left(G_{+}(D)\right) \cong K h^{p, q}(L)$. Additionally, for all $j \in \mathbb{Z}$, there is an isomorphism of torsion tor $H_{\mathcal{A}_{2}}^{\ell, j}\left(G_{+}(D)\right) \cong$ tor $K h^{\ell-c_{-}, q}(L)$.

Similarities between Khovanov link and chromatic homology go beyond this theorem, and extend mainly to alternating knots and their associated graphs. Note that the following result from [LS17] states that the portion of Khovanov homology of any link is the same as Khovanov homology of an alternating link provided that their associated graphs are isomorphic. More precisely, if $D$ is an alternating diagram of a link $L$ and $D^{\prime}$ is a diagram of any link $L$ such that $G=G_{+}(D)=G_{+}\left(D^{\prime}\right)$, then we have the following isomorphism of Khovanov homology groups: $K h^{i, j}(D) \cong K h^{p, q}\left(D^{\prime}\right)$ for $-c_{-}(D) \leq i \leq-c_{-}(D)-\ell(G)-1$ and all $j$ where $p-c_{-}\left(D_{1}\right)=i-c_{-}\left(D_{0}\right)$ and $q+c_{+}\left(D^{\prime}\right)-2 c_{-}\left(d^{\prime}\right)=$ $j+c_{+}(D)-2 c_{-}(D)[$ LS17, Cor. 5.2]. 


\section{Chapter 3}

\section{Structure of chromatic homology over $\mathcal{A}_{2}=\mathbb{Z}[x] /\left(x^{2}\right)$}

In this section we prove a number of results about the chromatic homology of a graph over $\mathcal{A}_{2}$, building on the results found in [HGR05, HGPR06, CCR08, LS17]. Using the long exact sequence (2.4) and a result about chromatic polynomials, we determine the homological span of $H_{\mathcal{A}_{2}}(G)$ for any connected graph $G$, showing that it depends only on the number of vertices and the number of blocks in the graph. We also use the long exact sequence together with the knight move structure described in Theorem 5 to describe the effect on homology when we glue a cycle to $G$ along a single edge or a single vertex. These results provide information about torsion in extreme gradings of Khovanov homology. For certain links, we can describe an interval of Khovanov gradings on which torsion has no gaps.

We provide explicit descriptions of chromatic homology in gradings $i=3$ through $i=6$, derived from the coefficients of the chromatic polynomial [Mer72, Far80, Bie05]. For homologically thin links which a corresponding graph $G$ with girth $\ell$, we show that the first $\ell$ coefficients of the Jones polynomial depend only on the cycles of $G$.

\subsection{Homological span}

In order to compute the precise homological span of $H_{\mathcal{A}_{2}}(G)$, we first observe that the minimal quantum grading is equal to the number of blocks in a graph, then define a contracting sequence of graphs that will induce inclusion between their corresponding homology groups.

A bridge of a connected graph $G$ is an edge $e \in E(G)$ such that $G-e$ is disconnected. We say that a graph is 2-connected if it cannot be disconnected by the removal of a single edge.

Definition 13. A subgraph $B$ is a block of $G$ (also known as a biconnected component of $G$ ) if it 
is either a bridge or a maximal 2-connected subgraph of $G$ ([Bol98]). We let $b=b(G)$ denote the number of blocks of $G$.

Proposition 5. [Rea68, WJZ84] Let $G$ be any graph and let $c_{0}, c_{1}$ be the multiplicities of the factors $\lambda,(\lambda-1)$ respectively in $P_{G}(\lambda)$. Then $c_{0}$ is the number of connected components in $G$ and $c_{1}$ is the number of blocks.

Lemma 1. Let $j_{\min }$ be the minimal quantum grading for which $H_{\mathcal{A}_{2}}^{*, j}(G)$ is non-trivial. Then $j_{\min }\left(H_{\mathcal{A}_{2}}^{*, j}(G)\right)=b(G)$.

Proof. Theorem 5 implies that there is only one non-trivial homology group in the minimal quantum grading: $H_{\mathcal{A}_{2}}^{v-j_{\min }-1, j_{\min }}(G)$ on the lower diagonal. Therefore the lowest degree term in the chromatic polynomial $P_{G}(1+q)$ equals \pm rk $H_{\mathcal{A}_{2}}^{v-j_{\min }-1, j_{\min }}(G) q^{j_{\text {min }}}$, and $q^{j_{\min }}$ divides $P_{G}(1+q)$. In terms of the original variable $\lambda=1+q$ this means that $j_{\text {min }}$ is the multiplicity of the factor $(\lambda-1)$ in $P_{G}(\lambda)$, which is known to be equal to the number of blocks $b$ by Proposition 5 .

Given a graph $G$, we define a sequence of graphs obtained by contracting certain edges of $G$. The requirements of Definition 14 are tailored to fit conditions in Theorems 8 and 9, where we use the long exact sequence (2.4) and results of [CCR08] for connected graphs. In particular, we avoid contracting bridges, as in that case $G-e$ is not connected and its chromatic homology is not thin.

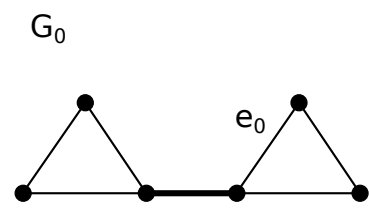

$\mathrm{G}_{1}$

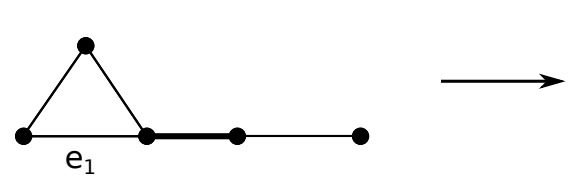

$\mathrm{G}_{2}$

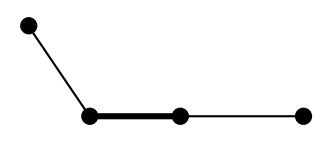

Figure 3.1: Contraction sequence $\left\{G_{0}, G_{1}, G_{2}\right\}$ with ending with a tree $G_{2}$.

Example 1. The contraction sequence shown in Figure 4 reduces the graph $G=G_{0}$ to a tree $G_{2}$ in $v(G)-b(G)-1=2$ steps. Note that bridges (represented by a bold line in $G_{0}$ ) can not be contracted, and remain fixed in the contraction sequence. First we reduce the block on the right to a single edge by contracting $e_{0}$ to obtain the first graph in the contracting sequence $G_{1}$. The second contracting step does the same for the block on the left by contracting $e_{1}$ which reduces $G_{1}$ to $G_{2}$ which is a tree.

Definition 14. A contraction sequence $G^{/ s}$ of a graph $G$ is a set of graphs $G^{/ s}=\left\{G_{i}\right\}_{i=0}^{n}$ such that $G_{0}=G$ and each $G_{i}$ with $0<i \leq n$ is obtained from $G_{i-1}$ by contracting a single non-bridge edge and removing any double edges after the contraction. 
Remark 1. Note that each contraction decreases the number of vertices in the graph by one; i.e., $v\left(G_{i}\right)=v\left(G_{i-1}\right)-1$. This procedure can never decrease the number of blocks because contraction of bridges is prohibited, and if a block has more than two vertices then any edge $e \in E(B)$ is contained in a cycle of $B$, so contraction of e can not eliminate B. Moreover, this procedure cannot remove cut-vertices, which implies that each block is contracted separately.

Lemma 2. For any graph $G$ there exists a contraction sequence $G^{/ s}$ that reduces $G$ to a tree in exactly $v-b-1$ steps, i.e. the first and only tree in a sequence $G^{/ s}$ is $\left\{G_{i}\right\}_{i \geq 0}$ is $G_{v-b-1}$.

Proof. In the light of Remark 1, we need to prove the existence of the longest possible contracting sequence because after $v-b-1$ steps we will have a graph with $b+1$ vertices and $b$ blocks so $G_{v-b-1}$ has to be a tree. Theorem 5.12 [Hav] states that if you have a 2-connected graph (in this case, a block) $\mathrm{B}$ with more than three vertices, there is an edge e of $\mathrm{B}$ such that $B / e$ is 2-connected. This fact ensures that we will not get a tree prior to $G_{v-b-1}$.

The tree obtained in Lemma 2 is similar to the "block-cutvertex tree" defined in [HP66] (see $[\operatorname{Bar} 02])$.

Theorem 8. For any connected graph $G$ with $v$ vertices and $b$ blocks, hspan $\left(H_{\mathcal{A}_{2}}(G)\right)=v-b$.

Proof. Since $H_{\mathcal{A}_{2}}^{0, v}(G)=\mathbb{Z}$ for any $G$, it suffices to show that the last nontrivial homology group occurs in homological grading $i=v-b-1$. In particular, we show that the group $H_{\mathcal{A}_{2}}^{v-b-1, b}(G)$ is non-trivial. Let $G^{/ s}$ be a contraction sequence described in Lemma 2. If $e_{1} \in E(G)$ is the first edge contracted in the sequence, we have a deletion-contraction long exact sequence in chromatic homology Eq. (2.4):

$$
\ldots \rightarrow H_{\mathcal{A}_{2}}^{v-b-2, b}\left(G-e_{1}\right) \rightarrow H_{\mathcal{A}_{2}}^{v-b-2, b}\left(G / e_{1}\right) \stackrel{\alpha_{7}}{\rightarrow} H_{\mathcal{A}_{2}}^{v-b-1, b}(G) \rightarrow \ldots
$$

in which $H_{\mathcal{A}_{2}}^{v-b-2, b}\left(G-e_{1}\right) \cong 0$ (because $G-e_{1}$ is connected and has $v$ vertices). Thus map $\alpha_{1}$ is injective. Applying the same argument to each of the steps in the contracting sequence yields:

$$
H_{\mathcal{A}_{2}}^{0, b}\left(G_{v-b-1}\right) \stackrel{\alpha_{v-b-1}}{\longrightarrow} \ldots \stackrel{\alpha_{3}}{\hookrightarrow} H_{\mathcal{A}_{2}}^{v-b-3, b}\left(\left(G / e_{1}\right) / e_{2}\right) \stackrel{\alpha_{2}}{\hookrightarrow} H_{\mathcal{A}_{2}}^{v-b-2, b}\left(G / e_{1}\right) \stackrel{\alpha_{1}}{\hookrightarrow} H_{\mathcal{A}_{2}}^{v-b-1, b}(G)
$$

with each $\alpha_{i}$ injective.

Since $G_{v-b-1}$ is a tree, based on Corollary $2, H_{\mathcal{A}_{2}}^{0, b}\left(G_{v-b-1}\right) \cong \mathbb{Z}$. The sequence of injections implies that $H_{\mathcal{A}_{2}}^{v-b-1, b}(G)$ is also non-trivial. So the span of homology on the $i+j=v-1$ diagonal is at least $v-b$. Since $H_{\mathcal{A}_{2}}^{v-j-1, j}(G)$ with $j<b$ must be trivial by Lemma 1 , the span is exactly $v-b$. Theorem 5 implies that the $i+j=v$ diagonal must have the same homological span. 
Theorem 9. Chromatic homology $H_{\mathcal{A}_{2}}^{i}(G)$ contains at least one copy of $\mathbb{Z}$ for each $i$-grading such that $0 \leq i \leq v-b-1$, that is, rk $H_{\mathcal{A}_{2}}^{i, v-i}(G) \oplus H_{\mathcal{A}_{2}}^{i, v-i-1}(G)>0$.

Proof. In case $i=0,1$ the statement follows from [PPS09, Thm. 3.1] and [PS14, Lem. 3.1].

Now let $2 \leq i \leq v-b-1$ and assume that $G$ is not a tree. The proof relies on the contraction sequence of Definition 14 and the deletion-contraction long exact sequence in chromatic homology. More precisely, we will show that the statement is true for all graphs in the contraction sequence, working backwards starting from $n=v-b-1$. By Lemma 2, there is a contraction sequence $\left\{G_{k}\right\}_{k=0}^{v-b-1}$ of $G$ such that $G_{v-b-1}$ is a tree. We let $G_{v-b-1}$ be our base case, since the result holds for any tree in homological degree zero (Corollary 2).

Next, assume that the result holds for $G_{k+1}, 1 \leq k+1 \leq v-b-1$. We show the result also holds for $G_{k}$.

In the induction step that follows, $v, E$, and $b$ refer to the number of vertices, edges, and blocks in $G_{k}$, respectively. By [CCR08]:

$$
\begin{aligned}
\operatorname{rk} H_{\mathcal{A}_{2}}^{i, v-i}\left(G_{k}\right) & =\operatorname{rk} H_{\mathcal{A}_{2}}^{i-1, v-i}\left(G_{k+1}\right)+\operatorname{rk} H_{\mathcal{A}_{2}}^{i, v-i}\left(G_{k}-e\right) \\
\operatorname{rk} H_{\mathcal{A}_{2}}^{i, v-i-1}\left(G_{k}\right) & =\operatorname{rk} H_{\mathcal{A}_{2}}^{i-1, v-i-1}\left(G_{k+1}\right)+\operatorname{rk} H_{\mathcal{A}_{2}}^{i, v-i-1}\left(G_{k}-e\right)
\end{aligned}
$$

where $e$ is the edge such that $G_{k+1}=G_{k} / e$.

Note that $G_{k+1}$ has $v-1$ vertices, $E-1$ edges, and $b$ blocks (the number of blocks cannot change since $e$ was not a bridge). The group $H_{\mathcal{A}_{2}}^{i-1, v-i}\left(G_{k+1}\right)$ is on the upper diagonal of the homology of $G_{k+1}$, while $H_{\mathcal{A}_{2}}^{i-v-i-1}\left(G_{k+1}\right)$ is immediately below it on the lower diagonal. Since $2 \leq i \leq v-b-1$, we have $1 \leq i-1 \leq v-b-2$ where $v-b-2=v\left(G_{k+1}\right)-b\left(G_{k+1}\right)-1$. By assumption, then, rk $H_{\mathcal{A}_{2}}^{i-1, v-i}\left(G_{k+1}\right) \oplus H_{\mathcal{A}_{2}}^{i-1, v-i-1}\left(G_{k+1}\right)>0$. This implies that rk $H_{\mathcal{A}_{2}}^{i-1, v-i}\left(G_{k+1}\right)>0$ or $\operatorname{rk} H_{\mathcal{A}_{2}}^{i-1, v-i-1}\left(G_{k+1}\right)>0$.

Using our description of homological span over $\mathcal{A}_{2}$, we can give a fairly weak lower bound for the span of torsion in Khovanov homology.

Theorem 10. Let $D$ be a link diagram of link $L$ whose graph $G_{+}(D)$ has $v$ vertices, $b$ blocks, and girth $\ell$.

$$
\operatorname{hspan}^{t}(K h(L)) \geq h s_{+}^{t}= \begin{cases}v-b-1 & G_{+}(D) \text { has odd cycle with } \ell \geq v-b-1 \\ v-b-2 & G_{+}(D) \text { is bipartite with } \ell \geq v-b-1 \\ \ell & G_{+}(D) \text { has odd cycle with } \ell<v-b-1 \\ \ell-1 & G_{+}(D) \text { is bipartite with } \ell<v-b-1\end{cases}
$$

Proof. The minimal $i$-grading with torsion is either $i=1$ (odd cycle) or $i=2$ (bipartite) [PPS09]. On the other hand, $H_{\mathcal{A}_{2}}(G)$ contains one $\mathbb{Z}_{2}$ in $(i+1, j-1)$ for each $(i, j),(i+1, j-2)$ knight move pair, based on the proof of Theorem 5 [LS17]. Therefore, the maximal homological grading with 
torsion is $i=v-b-1$, where the last $\mathbb{Z}$ occurs. If $\ell \geq v-b-1$, the last grading with torsion inside the correspondence is $i=v-b-1$ and the span of torsion is $v-b-1$ (odd cycle) or $v-b-2$ (bipartite). If $\ell<v-b-1$, then the span of torsion is at least $\ell$ (odd cycle) or $\ell-1$ (bipartite).

Corollary 3. Let $D$ be a link diagram whose graphs $G_{+}(D)$ and $G_{-}(D)$ have $v_{ \pm}$vertices, $b_{ \pm}$blocks, and girth $\ell_{ \pm}$, respectively. Using notation in Theorem 10 if both $h s_{+}^{t}, h s_{-}^{t}>0$ we know that the span of torsion relates to the homological span of Khovanov homology in the following way:

$$
2 \leq \operatorname{hspan}(K h(L))-\operatorname{hspan}^{t}(K h(L)) \leq 4 .
$$

\subsection{Addition of cycles}

In this section we analyze how attaching a cycle along an edge or vertex affects chromatic homology $H_{\mathcal{A}_{2}}(G)$ and use these results to describe patterns in Khovanov homology of some alternating 3strand pretzel links and rational 2-bridge links.

Recall that the chromatic homology of an $n$-cycle, denoted $P_{n}$, is determined by the Hochschild homology of the chosen algebra (Theorem 6). As mentioned before, we focus on polynomial algebras $\mathcal{A}_{m}$.

This result, applied to algebra $\mathcal{A}_{2}$, says the following:

Corollary 4. The chromatic homology for $P_{n}$ over $\mathcal{A}_{2}$ is given by

$$
\begin{aligned}
H_{\mathcal{A}_{2}}^{i, n-i}\left(P_{n=2 k+1}\right) \cong \begin{cases}\mathbb{Z}_{2} & i \text { odd }, 1 \leq i \leq n-2 \\
\mathbb{Z} & i \text { even }, 0 \leq i \leq n-3\end{cases} \\
H_{\mathcal{A}_{2}}^{i, n-i}\left(P_{n=2 k}\right) \cong \begin{cases}\mathbb{Z}_{2} & i \text { even }, 2 \leq i \leq n-2 \\
\mathbb{Z} & i=0 \text { or } i \text { odd }, 1 \leq i \leq n-3\end{cases} \\
H_{\mathcal{A}_{2}}^{i, n-i-1}\left(P_{n=2 k+1}\right) \cong \begin{cases}\mathbb{Z} & i \text { odd }, 1 \leq i \leq n-2 \\
0 & \text { otherwise }\end{cases} \\
H_{\mathcal{A}_{2}}^{i, n-i-1}\left(P_{n=2 k}\right) \cong \begin{cases}\mathbb{Z} & i \text { even, } 0 \leq i \leq n-2 \\
0 & \text { otherwise }\end{cases}
\end{aligned}
$$

For other connected graphs, explicit formulae were known only for the first three homological gradings [AP04, PPS09, PS14] and Theorem 21 describes the fourth grading. It is not surprising, but still curious, that these initial gradings in chromatic homology depend only on the bipartiteness and the number of triangles.

Definition 15. The cyclomatic number $p_{1}(G)$ of a connected graph $G$ is equal to $p_{1}(G)=|E|-v+1$. 
Proposition 6. [PPS09, PS14] Let $G$ be a graph, with $t_{1}$ equal to the number of triangles in $G$.

Then:

$$
\begin{aligned}
& H_{\mathcal{A}_{2}}^{0, v}(G)=\mathbb{Z} \quad H_{\mathcal{A}_{2}}^{1, v-1}(G)= \begin{cases}\mathbb{Z}^{p_{1}} & G \text { bipartite } \\
\mathbb{Z}^{p_{1}-1} \oplus \mathbb{Z}_{2} & \text { otherwise }\end{cases}
\end{aligned}
$$

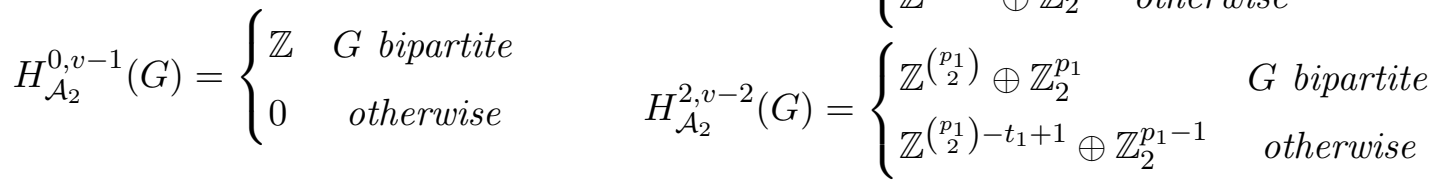

Lemma 3 states that entries on the main diagonal in chromatic homology of graph $G$ are determined by entries from the main diagonals of chromatic homology for graphs $G-e$ and $G / e$, provided that edge $e$ is not a bridge.

Lemma 3. Given graph $G$ with $v$ vertices and an edge $e \in E(G)$ which is not a bridge, then for all $i \geq 2$,

$$
H_{\mathcal{A}_{2}}^{i, v-i}(G) \cong H_{\mathcal{A}_{2}}^{i-1, v-i}(G / e) \oplus H_{\mathcal{A}_{2}}^{i, v-i}(G-e)
$$

Proof. The free part of $H_{\mathcal{A}_{2}}^{i, v-i}(G)$ is determined by computation of rational chromatic homology [CCR08, Corollary 4.2]:

$$
\operatorname{rk} H_{\mathcal{A}_{2}}^{i, v-i}(G ; \mathbb{Q})=\operatorname{rk} H_{\mathcal{A}_{2}}^{i-1, v-i}(G / e ; \mathbb{Q})+\operatorname{rk} H_{\mathcal{A}_{2}}^{i, v-i}(G-e ; \mathbb{Q})
$$

The same result, applied in the previous homological grading

$$
\operatorname{rk} H_{\mathcal{A}_{2}}^{i-1, v-i+1}(G ; \mathbb{Q})=\operatorname{rk} H_{\mathcal{A}_{2}}^{i-2, v-i+1}(G / e ; \mathbb{Q})+\operatorname{rk} H_{\mathcal{A}_{2}}^{i-1, v-i+1}(G-e ; \mathbb{Q})
$$

together with Theorem 5 determine the torsion on the main diagonal:

$$
\text { tor } H_{\mathcal{A}_{2}}^{i, v-i}(G)=\text { tor } H_{\mathcal{A}_{2}}^{i-1, v-i}(G / e) \oplus \text { tor } H_{\mathcal{A}_{2}}^{i, v-i}(G-e) \text {. }
$$

\subsubsection{Edge gluing of a cycle}

In this section we analyze how attaching a cycle along an edge or vertex affects chromatic homology $H_{\mathcal{A}_{2}}(G)$.

We use the notation $G_{1} \mid G_{2}$ to represent the graph obtained by gluing $G_{1}$ and $G_{2}$ along a single edge, and $\left.G_{1}\right|^{k} G_{2}$ for a gluing along $k$ edges, Figure 3.2. Similarly, $G_{1} * G_{2}$ is the gluing of $G_{1}$ and $G_{2}$ at a single vertex.

Theorem 11 provides an explicit formula for the upper diagonal $i+j=v$ of $H_{A_{2}}\left(G \mid P_{n}\right)$, and, together with Theorem 5, determines the rest of chromatic homology, i.e. the lower diagonal. 


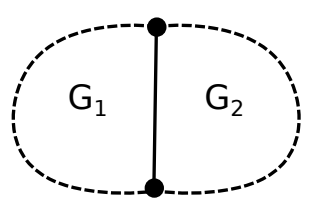

$\mathrm{G}_{1} \mid \mathrm{G}_{2}$

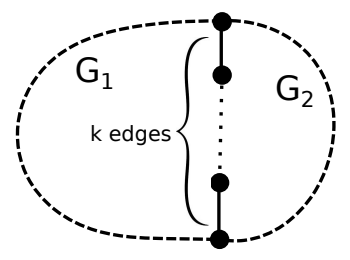

$\left.\mathrm{G}_{1}\right|^{\mathrm{k}} \mathrm{G}_{2}$

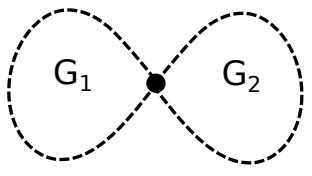

$\mathrm{G}_{1} * \mathrm{G}_{2}$

Figure 3.2: Edge and vertex gluings of graphs.

Theorem 11. Let $G$ be a graph with $v$ vertices, $E$ edges, and $S_{t}(G)=\bigoplus_{k=0}^{t} H_{\mathcal{A}_{2}}^{i-k, v-i+k}(G)$. For $n \geq 3$,

$$
H_{\mathcal{A}_{2}}^{i,(v+n-2)-i}\left(G \mid P_{n}\right) \cong \begin{cases}S_{n-2}(G) & i>n-2 \\ \mathbb{Z}^{E-v+2} \oplus S_{i-2}(G) & i \leq n-2, n-i \text { odd, G bipartite } \\ \mathbb{Z}^{E-v+1} \oplus \mathbb{Z}_{2} \oplus S_{i-2}(G) & \text { otherwise }\end{cases}
$$

Proof. Note that $H_{\mathcal{A}_{2}}^{i, v\left(G \mid P_{n}\right)-i}\left(G \mid P_{n}\right)=H_{\mathcal{A}_{2}}^{i,(v+n-2)-i}\left(G \mid P_{n}\right)$. First we consider the case where $i>$ $n-2$. We induct on $n$, the length of the added cycle. For $n=3$, let $e$ be an edge of $P_{3}$ that is not in $G$.

Observe that $\left(G \mid P_{3}\right) / e$ is $G$ with a double edge, so $H_{\mathcal{A}_{2}}\left(\left(G \mid P_{3}\right) / e\right) \cong H_{\mathcal{A}_{2}}(G)$. The graph $G \mid P_{3}-e$ is $G$ with a pendant edge. From Lemma 3 and Theorem 4, we obtain the proof for $n=3$ :

$$
H_{\mathcal{A}_{2}}^{i,(v+1)-i}\left(G \mid P_{3}\right) \cong H_{\mathcal{A}_{2}}^{i-1,(v+1)-i}\left(G \mid P_{3} / e\right) \oplus H_{\mathcal{A}_{2}}^{i,(v+1)-i}\left(G \mid P_{3}-e\right) \cong H_{\mathcal{A}_{2}}^{i-1,(v+1)-i}(G) \oplus H_{\mathcal{A}_{2}}^{i, v-i}(G)
$$

The induction step is based on following:

$$
\begin{aligned}
H_{\mathcal{A}_{2}}^{i,(v+n-2)-i}\left(G \mid P_{n}\right) & \cong H_{\mathcal{A}_{2}}^{i-1,(v+n-2)-i}\left(G \mid P_{n} / e\right) \oplus H_{\mathcal{A}_{2}}^{i,(v+n-2)-i}\left(G \mid P_{n}-e\right) \\
& \cong H_{\mathcal{A}_{2}}^{i-1,(v+n-2)-i}\left(G \mid P_{n-1}\right) \oplus H_{\mathcal{A}_{2}}^{i,(v+n-2)-i}(G)\{n-2\} \\
& \cong H_{\mathcal{A}_{2}}^{i-1,(v+n-3)-(i-1)}\left(G \mid P_{n-1}\right) \oplus H_{\mathcal{A}_{2}}^{i, v-i}(G) \\
& \cong\left(\bigoplus_{k=0}^{n-3} H_{\mathcal{A}_{2}}^{(i-1)-k, v-(i-1)+k}(G)\right) \oplus H_{\mathcal{A}_{2}}^{i, v-i}(G) \cong \bigoplus_{k=0}^{n-2} H_{\mathcal{A}_{2}}^{i-k, v-i+k}(G) .
\end{aligned}
$$

For cases where $i \leq n-2$, we state the result differently to accommodate the extra $\mathbb{Z}$ in bipartite graphs. We apply Lemma 3 a total of $i-1$ times to obtain: 


$$
\begin{aligned}
H_{\mathcal{A}_{2}}^{i,(v+n-2)-i}\left(G \mid P_{n}\right) & \cong H_{\mathcal{A}_{2}}^{i-1,(v+n-2)-i}\left(G \mid P_{n-1}\right) \oplus H_{\mathcal{A}_{2}}^{i, v-i}(G) \\
& \cong H_{\mathcal{A}_{2}}^{1,(v+n-2)-i}\left(G \mid P_{n-(i-1)}\right) \oplus \bigoplus_{k=0}^{i-2} H_{\mathcal{A}_{2}}^{i-k, v-i+k}(G)
\end{aligned}
$$

Now we compute the first summand in terms of $G$ only, using Proposition 6:

$$
\begin{aligned}
H_{\mathcal{A}_{2}}^{1,(v+n-2)-i}\left(G \mid P_{n-(i-1)}\right) & =H_{\mathcal{A}_{2}}^{1, v\left(G \mid P_{n-(i-1)}\right)-1}\left(G \mid P_{n-(i-1)}\right) \\
& = \begin{cases}\mathbb{Z}^{E-v+2} & G \mid P_{n-(i-1)} \text { is bipartite } \\
\mathbb{Z}^{E-v+1} \oplus \mathbb{Z}_{2} & \text { otherwise }\end{cases}
\end{aligned}
$$

Since $G \mid P_{n-(i-1)}$ is bipartite only for $G$ bipartite, $n-i$ odd, we have derived the formulas for the second and third cases.

The following results are special cases of the previous theorem when graph $G$ is also a cycle.

Corollary 5. The rank of $H_{\mathcal{A}_{2}}^{i, v-i}\left(P_{3} \mid P_{n}\right)$ is given by $\mathrm{rk} H_{\mathcal{A}_{2}}^{i, v-i}\left(P_{3} \mid P_{n}\right)=\left\{\begin{array}{lc}1 & 0 \leq i \leq n-2 \\ 0 & \text { otherwise }\end{array}\right.$

Corollary 6. The rank of $H_{\mathcal{A}_{2}}^{i, v-i}\left(P_{4} \mid P_{n}\right)$ is given by the following formulas:

If $n$ is even, then $\operatorname{rk} H_{\mathcal{A}_{2}}^{i, v-i}\left(P_{4} \mid P_{n}\right)= \begin{cases}2 & i<n-1 \text { odd } \\ 1 & i<n-1 \text { even, } i=n-1 \\ 0 & i \geq n\end{cases}$
If $n$ is odd, then $\operatorname{rk} H_{\mathcal{A}_{2}}^{i, v-i}\left(P_{4} \mid P_{n}\right)= \begin{cases}2 & 0<i<n-1 \text { even } \\ 1 & i<n-1 \text { odd, } i=0, i=n-1 \\ 0 & i \geq n\end{cases}$

Corollary 7. The rank of $H_{\mathcal{A}_{2}}^{i, v-i}\left(P_{5} \mid P_{n}\right)$ is given by rk $H_{\mathcal{A}_{2}}^{i, v-i}\left(P_{5} \mid P_{n}\right)= \begin{cases}1 & i=0,1, n-1, n \\ 2 & 1<i<n-1 \\ 0 & i>n\end{cases}$

Concatenation of a pair of sequences $a=\left(a_{1}, \ldots, a_{k}\right)$ and $b=\left(b_{1}, \ldots, b_{\ell}\right)$ is denoted by $a \cdot b=$ $\left(a_{1}, \ldots, a_{k}, b_{1}, \ldots, b_{\ell}\right)$. Let $a^{\prime}$ denote the sequence obtained from $a$ by removing its last element; let $\bar{a}$ represent the sequence obtained from a by reversing its order. The notation $(a)^{p}=a \cdot a \cdot \ldots \cdot a$ represents the constant sequence of length $p$. We introduce the following notation for special integer sequences, as in [Man14]: $A_{p}=(2,1,3,2,4,3, \ldots, p, p-1)$ and $C_{p}=(1,1,2,2,3,3, \ldots, p, p)$. 
Torsion in chromatic homology of graphs $G=P_{s} \mid P_{t}$ depends on the parity of $s$ and $t$. Writing $s=2 m$ or $s=2 m+1$ and $j=2 n$ or $j=2 n+1$, we denote $M=M(G)=\min \{m, n\}$.

Theorem 12. For all graphs of the form $G=P_{s} \mid P_{t}(s, t \geq 3)$, torsion in chromatic homology follows the pattern tor $H_{\mathcal{A}_{2}}^{i, v-i}(G)=\mathbb{Z}_{2}^{x_{i}}$ where $x_{i}$ is the ith term of the sequences $x=\left(x_{n}\right)_{n \in \mathbb{N}}$ described below:

A) If $G=P_{2 n+1} \mid P_{2 m+1}$ then $x=C_{M-1} \cdot(M)^{2|m-n|+2} \cdot \bar{C}_{M-1}$ for $1 \leq i \leq 2 n+2 m-2$.

B) If $G=P_{2 n+1} \mid P_{2 m}$ with $n \leq m$, then $x=C_{M-1} \cdot(M)^{2|m-n|+1} \cdot \bar{C}_{M-1}$ for $1 \leq i \leq 2 n+2 m-3$.

C) If $G=P_{2 n+1} \mid P_{2 m}$ with $n>m$, then $x=C_{M-1} \cdot M \cdot(M-1, M)^{|m-n|} \cdot \bar{C}_{M-1}$ for $1 \leq i \leq$ $2 n+2 m-3$.

D) If $G=P_{2 n} \mid P_{2 m}$, then $x=A_{M-1} \cdot M \cdot(M-1, M)^{|m-n|} \cdot \bar{C}_{M-1}$ for $1 \leq i \leq 2 n+2 m-4$.

Proof. Based on Theorem 5 [LS17] the torsion pattern follows from the the free part of homology on the $i+j=v$ diagonal.

We prove the result for all $P_{s} \mid P_{t}$ where $s \leq t$. This suffices because graphs $P_{s} \mid P_{t}$ and $P_{t} \mid P_{s}$ are isomorphic. The result holds for $P_{3} \mid P_{t}, t \geq 3$ (Corollary 5) and $P_{4} \mid P_{t}, t \geq 4$ (Corollary 6). It follows that the result holds for $P_{s} \mid P_{3}$ for any $s \geq 3$, and $P_{s} \mid P_{4}$ for any $s \geq 4$, - we use this as a base for the induction.

Next, fix $s \geq 5$ and assume the result holds for $P_{s} \mid P_{q}, q<s$. To show that it holds for $P_{s} \mid P_{q}$, $q \geq s$ we consider the following four cases based on the parity of cycle lengths:

A) Suppose $G=P_{s}\left|P_{q}=P_{2 n+1}\right| P_{2 m+1}$, with $M=\min \{m, n\}=n \leq m$. Let $e$ be an edge of $G$ that is contained in $P_{2 m+1}$ but not in the other cycle. Then $G / e=P_{2 n+1} \mid P_{2 m}$ and $G-e=P_{2 n+1}$ with $2 m$ pendant edges. By assumption, homology of $G / e$ follows the pattern given in case B): $C_{n-1} \cdot(n)^{2(m-n)+1} \cdot \bar{C}_{n-1}$. We have rk $H_{\mathcal{A}_{2}}^{0, v}(G)=1$ and $\operatorname{rk} H_{\mathcal{A}_{2}}^{1, v-1}(G)=1$ by Proposition 6. For $i>1$, Equation (3.1) gives:

\begin{tabular}{|c|c|c|c|c|c|}
\hline $12233 \ldots$ & $(n-2)$ & $(n-1)$ & $(n-1)$ & $\underbrace{n \ldots n}_{2(m-n)+1} \bar{C}_{n-1}$ & (homology of $G / e$ ) \\
\hline$+10101 \ldots$ & 1 & 0 & 1 & & (homology of $G-e$ ) \\
\hline$=2233 \ldots$ & $(n-1)$ & $(n-1)$ & $n$ & $\underbrace{n \ldots n}_{2(m-n)+1} \bar{C}_{n-1}$ & (homology of $G$ ) \\
\hline
\end{tabular}

The final pattern for $H_{\mathcal{A}_{2}}^{i, v-i}(G)$ is

$$
112233 \ldots \quad(n-1)(n-1) n \underbrace{n \ldots n}_{2(m-n)+1} \bar{C}_{n-1}=C_{n-1} \cdot(n)^{2(m-n)+2} \cdot \bar{C}_{n-1}
$$


B) Analogously, case $G=P_{s}\left|P_{q}=P_{2 n+1}\right| P_{2 m}, M=n \leq m$, builds off of case A). Choosing an edge $e \in G$ that is contained only in $P_{2 m}$ means that $G / e=P_{2 n+1} \mid P_{2(m-1)+1}$ and $G-e=$ $P_{2 n+1}$ with $2 m-1$ pendant edges attached.

C) Notice that $G=P_{s}\left|P_{q}=P_{2 n+1}\right| P_{2 m}, n>m$ is isomorphic to $G=P_{q}\left|P_{s}=P_{2 m}\right| P_{2 n+1}$. In this case $M=m$ and for simplicity of the argument, we choose the edge of the odd cycle which reduces the computation to graph $P_{2 m} \mid P_{2 n}$ which belongs to Case D).

D) Let $G=P_{s}\left|P_{q}=P_{2 n}\right| P_{2 m}$ with $M=\min \{m, n\}=n \leq m$. Select an edge $e$ of $G$ that is contained in $P_{2 m}$ but not in the other cycle. Then $G / e=P_{2 n} \mid P_{2(m-1)+1}$ and $G-e=P_{2 n}$ with pendant edges attached. Case $\mathrm{C}$ ) gives us the homology of $G / e$ if $n<m-1$; if $n=m$ or $n=m-1$, use Case B) instead.

The proof of the following theorem is omitted, as it closely follows the proof of Theorem 12 .

Theorem 13. For all graphs of the form $G=\left.P_{s}\right|^{2} P_{t}(s, t \geq 4)$, torsion in chromatic homology follows the pattern tor $H_{\mathcal{A}_{2}}^{i, v-i}(G)=\mathbb{Z}_{2}^{x_{i}}$ where $x_{i}$ is the ith term of the sequences $x=\left(x_{n}\right)_{n \in \mathbb{N}}$ described below:

A) If $G=\left.P_{2 n+1}\right|^{2} P_{2 m+1}$ with $M=\min \{m, n\}$, then $x=C_{M-1} \cdot(M)^{2|m-n|+2} \cdot \bar{C}_{M-1}^{\prime}$ for $1 \leq i \leq$ $2 n+2 m-3$.

B) If $G=\left.P_{2 n+1}\right|^{2} P_{2 m}$ with $n \leq m$, then $x=C_{M-1} \cdot(M)^{2|m-n|+1} \cdot \bar{C}_{M-1}^{\prime}$ for $1 \leq i \leq 2 n+2 m-4$.

C) If $G=\left.P_{2 n+1}\right|^{2} P_{2 m}$ with $n>m$, then $x=C_{M-1} \cdot M \cdot(M-1, M)^{|m-n|} \cdot \bar{C}_{M-1}^{\prime}$ for $1 \leq i \leq$ $2 n+2 m-4$.

D) If $G=\left.P_{2 n}\right|^{2} P_{2 m}$, then $x=A_{M-1} \cdot M \cdot(M-1, M)^{|m-n|} \cdot \bar{C}_{M-1}^{\prime}$ for $1 \leq i \leq 2 n+2 m-5$.

\subsubsection{Vertex gluing of a cycle}

Using ideas outlined in Section 3.2.1, we describe the chromatic homology of graphs obtained by gluing a cycle along a vertex of a given graph. These results allow us to give an alternative proof of [WW92, Theorem 2] stating that certain classes of outerplanar graphs are cochromatic.

Corollary 8, which follows from Theorem 11, says that gluing a cycle to $G$ at a vertex has the same effect as gluing along a single edge, up to a shift in the $j$-grading.

Corollary 8. For any graph $G$ and any $n \geq 3, H_{\mathcal{A}_{2}}^{i, v-i}\left(G * P_{n}\right)=H_{\mathcal{A}_{2}}^{i, v-i-1}\left(G \mid P_{n}\right)$. 
Proof. The proof is analogous to the proof of Theorem 11, and yields:

$$
\begin{aligned}
& H_{\mathcal{A}_{2}}^{i, v\left(G * P_{n}\right)-i}\left(G * P_{n}\right) \cong H_{\mathcal{A}_{2}}^{i,(v+n-1)-i}\left(G * P_{n}\right) \\
& \cong \begin{cases}S_{n-2}(G) & i>n-2 \\
\mathbb{Z}^{E-v+2} \oplus S_{i-2}(G) & i \leq n-2, n-i \text { odd, } G \text { bipartite } \\
\mathbb{Z}^{E-v+1} \oplus \mathbb{Z}_{2} \oplus S_{i-2}(G) & \text { otherwise }\end{cases}
\end{aligned}
$$

Since $G * P_{n}$ has one more vertex than $G \mid P_{n}$, the formula above implies that $G * P_{n}$ has the same homology as $G \mid P_{n}$ with an upward shift of one $j$-grading.

The results in this section determine the chromatic homology of graphs constructed iteratively by gluing cycles only along single edges, or along both single edges and vertices. These families of graphs are known as polygon trees and outerplanar graphs, respectively.

Definition 16. A first-order polygon tree is a graph consisting of a single cycle. An nth order polygon tree may be constructed by gluing a new cycle along one edge of an $(n-1)$ st order polygon tree.

Definition 17. A planar graph is outerplanar if it can be embedded in the plane with all its vertices on the same face.

Remark 2. The set of outerplanar graphs may be considered a generalization of polygon trees in which cycles are glued along a single edge, glued at a single vertex, or connected by a bridge. An equivalent description is given in [Sys79, Theorem 4].

Theorem 14. Suppose that $G=G_{1} * G_{2}$ and $G_{B}$ is the graph obtained by expanding the shared vertex into a bridge between $G_{1}$ and $G_{2}$. Then $H_{\mathcal{A}_{2}}\left(G_{B}\right)=H_{\mathcal{A}_{2}}(G)\{1\}$.

Proof. Let $J_{1}$ denote $G_{1}$ with a pendant edge, $J_{2}$ denote $G_{2}$ with a pendant edge. A result for chromatic polynomials ([DKT05], [Zyk49]) says that: $P_{G}(\lambda)=\frac{P_{G_{1}}(\lambda) P_{G_{2}}(\lambda)}{\lambda}$ and

$$
P_{G_{B}}(\lambda)=\frac{P_{J_{1}}(\lambda) P_{J_{2}}(\lambda)}{\lambda(\lambda-1)}=\frac{\left((\lambda-1) P_{G_{1}}(\lambda)\right)\left((\lambda-1) P_{G_{2}}(\lambda)\right)}{\lambda(\lambda-1)}=(\lambda-1) P_{G}(\lambda) .
$$

Changing variables to $q=\lambda-1$, we have $P_{G_{B}}(q)=q P_{G}(q)$, so $H_{\mathcal{A}_{2}}\left(G_{B}\right)$ and $H_{\mathcal{A}_{2}}(G)$ are determined up to a shift of one $q$-grading.

Definition 18. An induced subgraph $H \subseteq G$ is a graph such that $V(H) \subseteq V(G)$ and $E(H)$ contains all edges in $E(G)$ with both endpoints in $H$. 
Note that induced cycles of $G$ are sometimes referred to as "chordless cycles" or "pure cycles".

If two polygon-trees have the same collection of induced cycles, they are chromatically equivalent; i.e., they have the same chromatic polynomial ([CL85], [WW92]). An analogous result holds for outerplanar graphs with the same collection of induced cycles and the same number of blocks [WW92, Theorem 2]. Corollary 9 provides another proof of this fact using chromatic homology.

Corollary 9. [WW92, Theorem 2] The family of all connected outerplanar graphs with $r_{k}$ induced cycles of length $k$ and $b$ blocks is chromatically equivalent. If $G$ is in this family, and $G^{E}$ is a polygon tree with the same collection of induced cycles, then $P_{G}(\lambda)=(\lambda-1)^{y} P_{G^{E}}(\lambda)$ where $y$ is the total number of vertex gluings and bridges in $G$.

Proof. Based on Remark 2, we need to know the effect that gluing two cycles along a single edge, gluing two cycles at a vertex, or connecting two cycles by a bridge has on chromatic graph homology. Theorem 11, Corollary 8, and Theorem 14 cover all relevant graph operations.

Corollary 10. Let $G$ be a connected outerplanar graph with $r_{k}$ induced cycles of length $k$. Then $\operatorname{hspan}\left(H_{\mathcal{A}_{2}}(G)\right)=\sum r_{k}(k-2)+1$.

Proof. Follows from [WW92, Theorem 2] and our considerations.

\subsubsection{Khovanov homology of certain 3-strand pretzel links}

Note that the graphs $G \mid P_{n}$ described in Subsection 3.2.1 are instances of multibridge graphs, Figure 3.3, defined as follows:

Definition 19 ([DHK $\left.\left.{ }^{+} 04\right]\right)$. The multibridge graph $\theta\left(a_{1}, a_{2}, \ldots, a_{k}\right)$ is the graph obtained by connecting two distinct vertices with $k$ internally disjoint paths, each of length $a_{k}$. In particular, $\theta\left(a_{1}, a_{2}, \ldots, a_{k}\right)$ is called a $k$-bridge graph.

Specifically, the 3-bridge graph $\theta\left(a_{1}, 1, a_{2}\right)$ consists of two cycles $P_{a_{1}+1}$ and $P_{a_{2}+1}$ glued along a single edge. We will compute torsion patterns in chromatic homology of multibridge graphs of the form $P_{n} \mid{ }^{k} P_{m}$ when $k=1$ or $k=2$. Note that the graph assigned to the standard diagram of the pretzel knot $K=\left(-a_{1},-a_{2}, \ldots,-a_{n}\right)$, which is $\left.\left.\left.P_{a_{1}+a_{2}}\right|^{a_{2}} P_{a_{2}+a_{3}}\right|^{a_{3}} \ldots\right|^{a_{n-1}} P_{a_{n-1}+a_{n}}$, where $n \geq 3$ and $a_{i} \geq 2$ for all $i$, is precisely the multibridge graph $\theta\left(a_{1}, a_{2}, \ldots, a_{k}\right)$. As a corollary, we will be able to partially describe Khovanov homology of alternating 3 -strand pretzel knots.

For thin pretzel links, such as those which are alternating or quasi-alternating ([OS08], [Gre10]), torsion is determined by the Jones polynomial and signature via results of Alex Shumakovitch that inspired results in [LS17]. Three-strand pretzel links of the form $\left(p_{1}, p_{2},-q\right)$ are quasi-alternating if and only if $q>\min \left\{p_{1}, p_{2}\right\}$ [Gre10]. Rational Khovanov homology of $(p, q,-q)$ is given by a recursive formula on the parameter $p$ [Sta12, Qaz11]. Furthermore, links of the form $(p, q,-q)$ 

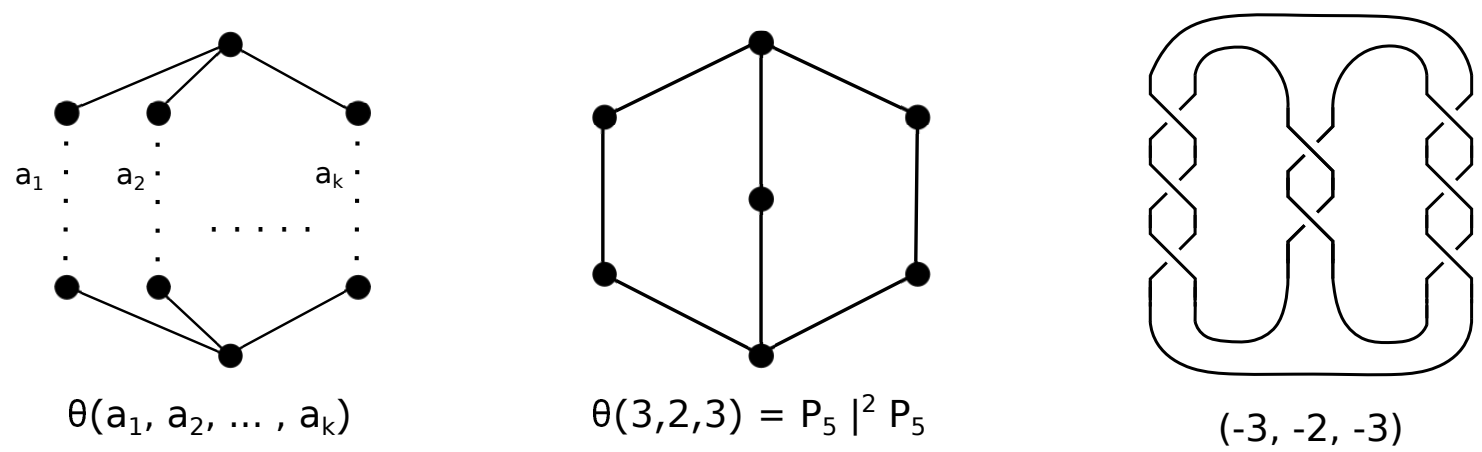

Figure 3.3: Multibridge graphs (left), multibridge graph $\theta(3,2,3)$ (middle) which can be seen as gluing two pentagons along two edges, that corresponds to the standard diagram of pretzel knot $(-3,-2,-3)$ (right).

with $q$ odd and $p>q$ are the only non-quasi-alternating pretzels which are homologically thin [Man13]. The results below describe patterns in Khovanov torsion of alternating links, in terms of the combinatorial properties of the corresponding graph.

Theorem 15. Let $L=\left(-a_{1}, \ldots,-a_{n}\right)$ be a pretzel link with standard diagram $D$. The homological span of torsion in $K h(L)$ has the following lower bound:

$$
\operatorname{hspan}^{t}(K h(L)) \geq \begin{cases}\min _{1 \leq i<j \leq n}\left\{a_{i}+a_{j}\right\}-1 & \text { if } a_{i}+a_{j} \text { is even for all } i \neq j \\ \min _{1 \leq i<j \leq n}\left\{a_{i}+a_{j}\right\} & \text { otherwise }\end{cases}
$$

Proof. This result is an application of Theorem 10 in the case of alternating pretzel knots. $G_{D}$ is a graph with 1 block and $\left(\sum_{i=1}^{n} a_{i}\right)-n+2$ vertices. By Theorem $8, \operatorname{hspan}\left(H_{\mathcal{A}_{2}}\left(G_{D}\right)\right)=\left(\sum_{i=1}^{n} a_{i}\right)-$ $n+1$. So the last torsion group occurs in grading $i=\left(\sum_{i=1}^{n} a_{i}\right)-n$ ([LS17]). To prove the result, we need to show that $\left(\sum_{i=1}^{n} a_{i}\right)-n$ is greater than or equal to the girth $l$.

The girth of a multibridge graph $G_{D}=\theta\left(a_{1}, a_{2}, \ldots, a_{n}\right)$ is $l=\min _{1 \leq i<j \leq n}\left\{a_{i}+a_{j}\right\}$. Without loss of generality, assume that $\ell=a_{j_{1}}+a_{j_{2}}$ and notice that

$$
\left(\sum_{i=1}^{n} a_{i}\right)-n=\sum_{i=1}^{n}\left(a_{i}-1\right)=\left(\sum_{i \neq j_{1}, j_{2}}\left(a_{i}-1\right)\right)+\left(a_{j_{1}}-1\right)+\left(a_{j_{2}}-1\right) \geq a_{j_{1}}+a_{j_{2}}
$$

The last inequality is true when $\sum_{i \neq j_{1}, j_{2}}\left(a_{i}-1\right) \geq 2$. This is true for any set of parameters except for $(-2,-2,-2)$ (this case can be verified by direct computation).

For thin links, torsion in Khovanov homology is determined by the Jones polynomial and signature [Shu16]. No general formula is known for computing this torsion. Using patterns in chromatic 
homology of multibridge graphs, we can describe a large part of $\mathbb{Z}_{2}$ torsion in the Khovanov homology of alternating pretzel links.

Example 2 (Torsion of pretzel knot and multibridge graph). The alternating knot with diagram $D=(-3,-2,-3)$, shown in Figure 3.3, is the mirror of $8_{5}$ in Rolfsen's table [Rol03, Kat]. Its corresponding graph is $G=\theta(3,2,3)$, which has girth 5 . In Table 3.1 we compare torsion in $K h(D)$ and $H_{\mathcal{A}_{2}}(G)$, using boldface to denote matching copies of $\mathbb{Z}_{2}$.

Table 3.1: Torsion in Khovanov homology of pretzel knot $L=(-3,-2,-3)$ and in chromatic homology of the corresponding graph $G=\theta(3,2,3)$. Entries in boldface denote the range where torsion is isomorphic.

\begin{tabular}{|l|l|l|l|l|l|l|l|l|l|}
\hline & $p=-5$ & $p=-4$ & $p=-3$ & $p=-2$ & $p=-1$ & $p=0$ & $p=1$ & $p=2$ \\
\hline tor $K h^{p}(L)$ & $\mathbb{Z}_{\mathbf{2}}$ & $\mathbb{Z}_{\mathbf{2}}$ & $\mathbb{Z}_{\mathbf{2}}^{\mathbf{2}}$ & $\mathbb{Z}_{\mathbf{2}}^{\mathbf{2}}$ & $\mathbb{Z}_{\mathbf{2}}$ & $\mathbb{Z}_{2}^{2}$ & & $\mathbb{Z}_{2}$ \\
\hline & $i=1$ & $i=2$ & $i=3$ & $i=4$ & $i=5$ & $i=6$ & $i=7$ & $i=8$ \\
\hline & $\mathbb{Z}_{\mathbf{2}}$ & $\mathbb{Z}_{\mathbf{2}}$ & $\mathbb{Z}_{\mathbf{2}}^{\mathbf{2}}$ & $\mathbb{Z}_{\mathbf{2}}^{\mathbf{2}}$ & $\mathbb{Z}_{\mathbf{2}}$ & & & \\
\hline
\end{tabular}

The following results are corollaries of the results in Section 3.2 that describe patterns in chromatic homology of multibridge graphs.

Corollary 11. Let $L$ be an alternating 3-strand pretzel link with diagram $D$ such that $D$ has $c_{-}$ negative crossings and $c_{+}$positive crossings and $G_{+}(D)$ has $v$ vertices. Then $L$ has torsion in Khovanov grading $\left(i-c_{-}, v-2 j+c_{+}-2 c_{-}\right)$equal to $\mathbb{Z}_{2}^{x_{i}}$ where $x_{i}$ is the ith term of the sequences $x$ described below:

A) If $D=(-(2 n-1),-2,-(2 m-1))$ with $m \neq n$, then $x=C_{M-1} \cdot M \cdot M \cdot M$ for $1 \leq i \leq 2 M+1$, where $M=\min \{m, n\}$.

B) If $D=(-(2 n-1),-2,-(2 n-1))$, then $x=C_{M-1} \cdot M \cdot M \cdot(M-1)$ for $1 \leq i \leq 2 n+1$.

C) If $D=(-(2 n-1),-2,-(2 m-2))$ with $n<m$, then $x=C_{M-1} \cdot M \cdot M \cdot M$ for $1 \leq i \leq 2 n+1$.

D) If $D=(-(2 n-1),-2,-(2 m-2))$ with $n \geq m$, then $x=C_{M-1} \cdot M \cdot(M-1)$ for $1 \leq i \leq 2 m$.

E) If $D=(-(2 n-2),-2,-(2 m-2))$, then $x=A_{M-1} \cdot M \cdot(M-1)$ for $1 \leq i \leq 2 M$.

If we take the graph $\theta\left(a_{1}, a_{2}, a_{3}\right)$ with a single parameter $a_{i}=1$, the corresponding alternating diagram describes a rational 2-bridge link. The Khovanov homology of these links is similar to that 
of the pretzel links above. Note that the sequences $C_{k}$ and $A_{k}$ in Corollaries 11 and 12 also appear in the rational homology of non-alternating pretzels [Man14].

Corollary 12. Let $L$ be a rational link with Conway notation $-P Q$ and diagram $D$ with $c_{-}$ negative crossings and $c_{+}$positive crossings. Let $v$ the number of vertices in $G_{+}(D)$. Then $L$ has torsion in Khovanov grading $\left(i-c_{-}, v-2 j+c_{+}-2 c_{-}\right)$equal to $\mathbb{Z}_{2}^{x_{i}}$ where $k_{i}$ is the ith term of the sequences $x$ described below:

A) If $L$ has Conway notation $-(2 n+1) 2 m+1$ with $m \neq n$, then $x=C_{M-1} \cdot M \cdot M \cdot M$ for $1 \leq i \leq 2 M+1$, where $M=\min \{m, n\}$.

B) If $L$ has Conway notation - $(2 n+1) 2 n+1$, then $x=C_{M-1} \cdot M \cdot M \cdot(M-1)$ for $1 \leq i \leq 2 n+1$.

C) If $L$ has Conway notation $-(2 n+1) 2 m$ with $n<m$, then $x=C_{M-1} \cdot M \cdot M \cdot M$ for $1 \leq i \leq 2 n+1$.

D) If $L$ has Conway notation $-(2 n+1) 2 m$ with $n \geq m$, then $x=C_{M-1} \cdot M \cdot(M-1)$ for $1 \leq i \leq 2 m$.

E) If $L$ has Conway notation $-2 n 2 m$, then $x=A_{M-1} \cdot M \cdot(M-1)$ for $1 \leq i \leq 2 M$.

\subsection{Existence of gaps in Khovanov and chromatic homology}

We prove several results concerning gaps in torsion for $H_{\mathcal{A}_{2}}(G)$ and their analogues for Khovanov homology of corresponding diagrams via Theorem 7 .

Definition 20. Let $H$ be either Khovanov or chromatic homology. A homological torsion gap of $H$ of length $g$ exists if there exists $i$ in the span of homology such that $H^{i-1}(G)$ and $H^{i+g}(G)$ has torsion, but $H^{k}(G)$ does not for $i \leq k<i+g$.

Notice that the quantum torsion gap can be defined analogously and that for chromatic homology over $\mathcal{A}_{2}$ and thin Khovanov homology, a homological gap in torsion is necessarily a quantum torsion gap, since torsion exists only on one diagonal.

Since there is a single $\mathbb{Z}_{2}$ in $H_{\mathcal{A}_{2}}^{1, v-1}(G)$ if $G$ has an odd cycle, and no torsion if $G$ is bipartite, the following definition involves homology in degrees two and higher.

Definition 21. Torsion of chromatic homology $H_{\mathcal{A}_{2}}(G)$ over algebra $\mathcal{A}_{2}$ is said to be dense if there is at least one $\mathbb{Z}_{2}$ in every $i$-grading from $i=2$ to $i=v-b-1$, i.e. if there are no homological torsion gaps.

Theorem 16. Chromatic homology $H_{\mathcal{A}_{2}}\left(P_{m} \mid P_{n}\right)$ of two polygons $P_{n}, P_{m}$ for $m, n \geq 3$ glued along an edge has dense torsion. 
Proof. Having dense torsion means that $H_{\mathcal{A}_{2}}^{i,(m+n-2)-i}\left(P_{m} \mid P_{n}\right)$ contains torsion for every $2 \leq i \leq$ $m+n-4$. Based on Theorem 11 we consider the following cases.

For $n-2<i \leq m+n-4$, we use the formula $H_{\mathcal{A}_{2}}^{i,(m+n-2)-i}\left(P_{m} \mid P_{n}\right) \cong \bigoplus_{k=0}^{n-2} H_{\mathcal{A}_{2}}^{i-k, m-i+k}\left(P_{m}\right)$. Since $n \geq 3$, the sum in the formula must include at least the $k=n-2$ and $k=n-3$ terms. That means we are looking into one of the $H_{\mathcal{A}_{2}}^{i^{*}, m-i^{*}}\left(P_{m}\right)$ with $i^{*}=i-k, 2 \leq k \leq m-2$ which must contain a copy of $\mathbb{Z}_{2}$ by Corollary 4 . Suppose $i \leq n-2, n-i$ is odd, and $m$ is even. The corresponding formula from Theorem $11 H_{\mathcal{A}_{2}}^{i,(m+n-2)-i}\left(P_{m} \mid P_{n}\right) \cong \mathbb{Z}^{E-v+2} \oplus \underset{k=0}{i-2} H_{\mathcal{A}_{2}}^{i-k, m-i+k}\left(P_{m}\right)$ contains $H_{\mathcal{A}_{2}}^{2, m-2}\left(P_{m}\right)=\mathbb{Z}_{2}$ for $k=i-2$.

For all other $i \leq n-2$ not covered by case 2 , there is $\mathbb{Z}_{2}$ torsion by the third formula in Theorem 11.

Corollary 13. If $G$ is a polygon-tree, then $H_{\mathcal{A}_{2}}(G)$ has dense torsion.

Proof. We induct on the number of cycles in $G$ with Theorem 16 as our base case for a graph $G_{2}$ with only two cycles. Assume the result holds for all polygon-trees with $p-1$ cycles, where $p \geq 3$. For the induction step we show that if $G_{p-1}$ is one such graph, then the result also holds for any $G_{p}=G_{p-1} \mid P_{n}, n \geq 3$.

If $v$ denotes the number of vertices in $G_{p-1}$ then $G_{p}$ has $v+n-2$ vertices and the gradings of interest are $2 \leq i \leq v+n-4$. According to Theorem 11 there are three cases.

For $n-1 \leq i \leq v+n-4$, Theorem 11 yields $H_{\mathcal{A}_{2}}^{i,(v+n-2)-i}\left(G_{p}\right) \cong \bigoplus_{k=0}^{n-2} H_{\mathcal{A}_{2}}^{i-k, v-i+k}\left(G_{p-1}\right)$. As in the proof of Theorem 16 we show that there exists a term that contributes at least one copy of $\mathbb{Z}_{2}$. By induction hypothesis, if $i=n-1$, then the term with $k=n-3$ contains torsion, otherwise the same is true for $k=n-2$. Similar arguments apply in the remaining two cases.

The following two Theorems are based on Corollary 8 and Corollary 13, respectively.

Theorem 17. If $G$ is a connected outerplanar graph, $H_{\mathcal{A}_{2}}(G)$ has dense torsion.

Theorem 18. If $H_{\mathcal{A}_{2}}(G)$ has dense torsion, the same is true of $H_{\mathcal{A}_{2}}\left(G \mid P_{n}\right)$ and $H_{\mathcal{A}_{2}}\left(G * P_{n}\right)$ for $n \geq 3$.

As a corollary we get the existence of $\mathbb{Z}_{2}$ torsion in Khovanov homology of some link provided that it can be associated a graph with certain properties.

Corollary 14. Let $D$ be a diagram of link $L$ such that $G=G_{+}(D)$ is a polygon-tree or bridgefree outerplanar graph where $v, b, \ell$ are the number of vertices, number of blocks, and girth of $G$. Then there is $\mathbb{Z}_{2}$ torsion in Khovanov homology $K h^{p, p-v+c_{+}-c_{-}}(L)$ of the corresponding link for $2-c_{-} \leq p \leq \min \{\ell, v-b-1\}-c_{-}$. 
Theorem 19. Let $L$ be an alternating 3-strand pretzel link with a diagram $D$ given by Conway symbol $-2,-2,-(n-2)$ where $n \geq 4$. Then there is a homological gap in torsion of its Khovanov homology $K h(L)$.

Proof. Note that diagram $D$ corresponds to a multibridge graph $G_{+}(D)=\theta(2, n-2,2)$ which has 1 block, $n+1$ vertices and girth $n$. By Theorem $8, H_{\mathcal{A}_{2}}\left(G_{+}(D)\right)$ has no homology in grading $i=n$, so $K h^{p, q}(L)$ has no torsion in the corresponding Khovanov grading $p=n-c_{-}$.

\subsection{Khovanov homology groups and the corresponding coefficients of the Jones polynomial}

Chromatic graph homology over algebra $\mathcal{A}_{2}$ has proven to be useful for providing explicit formulae for the first few extremal homological gradings of Khovanov homology subject to combinatorial conditions on the Kauffman state of a link diagram. The torsion groups in chromatic homology in degrees $i, v-i$ for $i=1,2,3$, are computed explicitly in [AP04, PPS09, PS14] and used to get the following gradings in Khovanov homology when the isomorphism theorem holds.

Proposition 7 ([PPS09, PS14]). Let $D$ be a diagram of $L$ with $c_{+}$positive crossings and $c_{-}$negative crossings, and let $G=G_{+}(D)$ with $p_{1}$ the cyclomatic number and $t_{1}$ the number of triangles.

$$
\begin{aligned}
& K h^{-c_{-},-v+c_{+}-2 c_{-}}(L)=\mathbb{Z} \\
& K h^{-c_{-},-v+2+c_{+}-2 c_{-}}(L)=\left\{\begin{array}{lc}
\mathbb{Z} & G \text { bipartite } \\
0 & \text { otherwise }
\end{array}\right. \\
& K h^{1-c_{-},-v+2+c_{+}-2 c_{-}}(L)= \begin{cases}\mathbb{Z}^{p_{1}} & \text { G bipartite } \\
\mathbb{Z}^{p_{1}-1} \oplus \mathbb{Z}_{2} & \text { otherwise }\end{cases}
\end{aligned}
$$

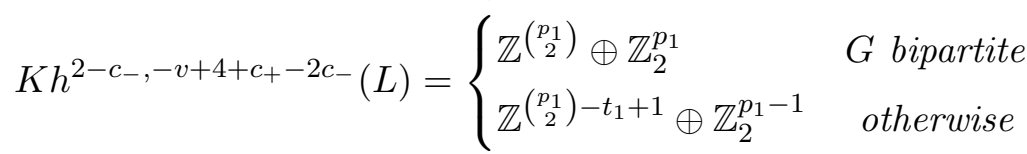

We use recent results from [LS17] and the formulas for coefficients of $P_{G}(\lambda)$ given in [Far80, Bie05], to calculate the torsion in the first $\ell(G)+1$ gradings of Khovanov homology.

Theorem 20. [Far80, Bie05] Let $G$ be a graph with $v$ vertices, $E$ edges, $t_{1}$ triangles, $t_{2}$ induced 4-cycles, and $t_{3}$ complete graphs of order 4. The first four coefficients of the chromatic polynomial

$$
P_{G}(\lambda)=c_{v} \lambda^{v}+c_{v-1} \lambda^{v-1}+c_{v-2} \lambda^{v-2}+c_{v-3} \lambda^{v-3}+\ldots
$$

are given by the following formulas: $c_{v}=1, c_{v-1}=-E, c_{v-2}=\left(\begin{array}{c}E \\ 2\end{array}\right)-t_{1}$, and $c_{v-3}=-\left(\begin{array}{c}E \\ 3\end{array}\right)+$ 
$(E-2) t_{1}+t_{2}-2 t_{3}$.

The 5th and 6th coefficients are given by the following formulas, where $t_{i}$ is the number of induced subgraphs of $G$ isomorphic to $T_{i}$ (see Figures 3.4, 3.5).

$$
\begin{aligned}
c_{v-4} & =\left(\begin{array}{c}
E \\
4
\end{array}\right)-\left(\begin{array}{c}
E-2 \\
2
\end{array}\right) t_{1}+\left(\begin{array}{c}
t_{1} \\
2
\end{array}\right)-(E-3) t_{2}-(2 E-9) t_{3}-t_{4}+t_{5}+2 t_{6}+3 t_{7}-6 t_{8} \\
c_{v-5} & =-\left(\begin{array}{c}
E \\
5
\end{array}\right)+\left(\begin{array}{c}
E-2 \\
3
\end{array}\right) t_{1}-(E-4)\left(\begin{array}{c}
t_{1} \\
2
\end{array}\right)+\left(\begin{array}{c}
E-3 \\
2
\end{array}\right) t_{2}-\left(t_{2}-2 t_{3}\right) t_{1}-\left(E^{2}-10 E+30\right) t_{3}+t_{4} \\
& -(E-3) t_{5}-2(E-5) t_{6}-3(q-6) t_{7}+6(E-8) t_{8}+t_{9}-t_{10}-2 t_{11}-2 t_{12}-t_{13}+t_{14} \\
& -t_{15}-3 t_{16}-4 t_{17}-4 t_{18}+2 t_{19}-4 t_{20}-t_{21}+4 t_{22}+3 t_{23}+4 t_{24}+5 t_{25}+4 t_{26}+6 t_{27}+8 t_{28} \\
& +16 t_{29}+12 t_{30}-24 t_{31}
\end{aligned}
$$

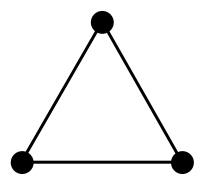

$T_{1}$

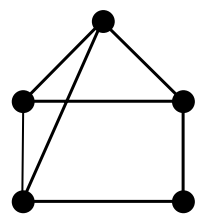

$T_{6}$

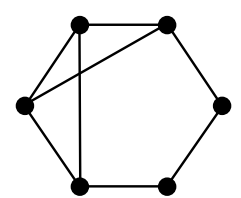

$T_{11}$

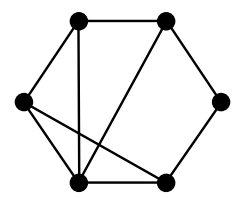

$T_{16}$

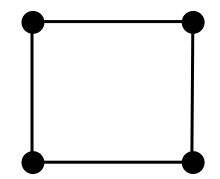

$T_{2}$

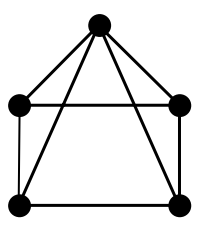

$T_{7}$

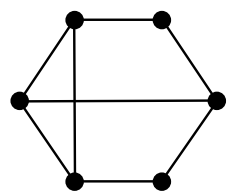

$T_{12}$

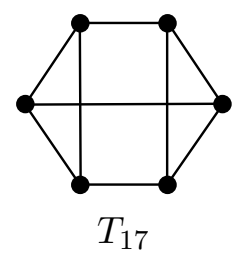

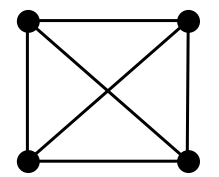

$T_{3}$

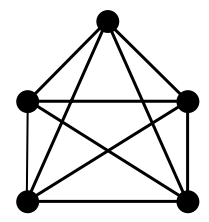

$T_{8}$

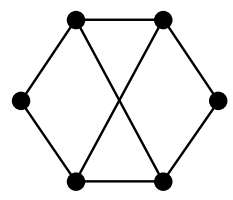

$T_{13}$

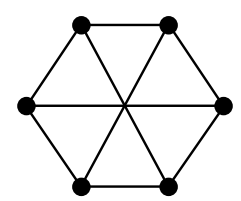

$T_{18}$

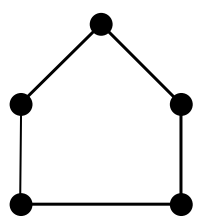

$T_{4}$

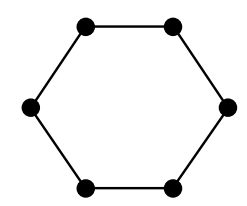

$T_{9}$

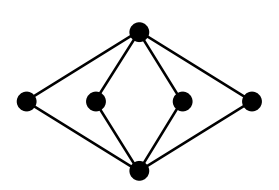

$T_{14}$

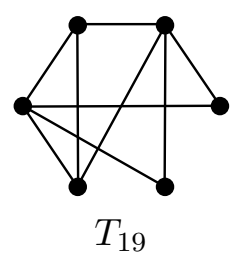

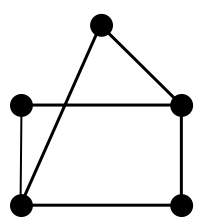

$T_{5}$

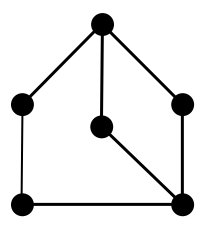

$T_{10}$

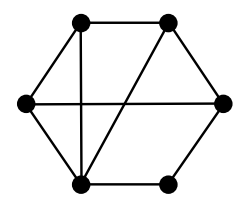

$T_{15}$

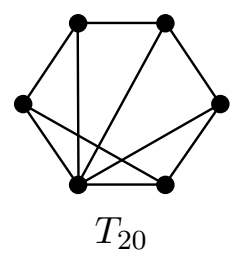

Figure 3.4: Graphs $T_{1}$ through $T_{20}$ involved in the computation of the 5 th and 6 th coefficients of the chromatic polynomial [Bie05]. 


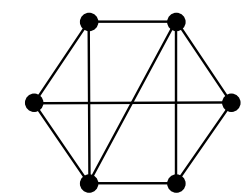

$T_{21}$

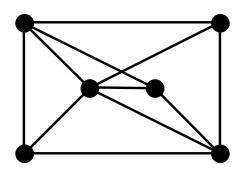

$T_{26}$

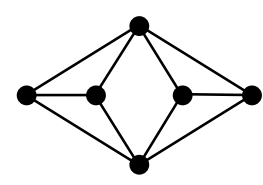

$T_{22}$

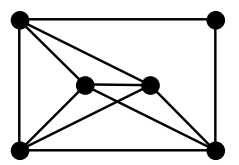

$T_{27}$

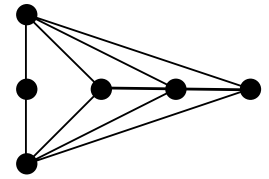

$T_{23}$

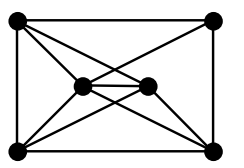

$T_{28}$

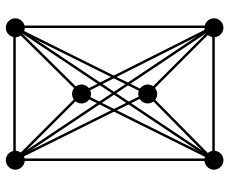

$T_{31}$

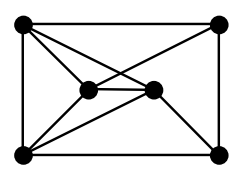

$T_{24}$

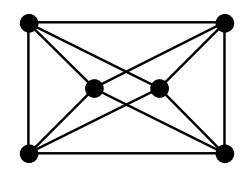

$T_{29}$
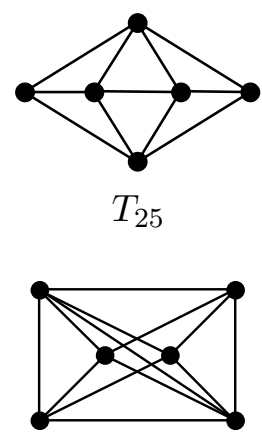

$T_{30}$

Figure 3.5: Graphs $T_{21}$ through $T_{31}$ involved in the computation of the 5 th and 6 th coefficients of the chromatic polynomial [Bie05].

The following result describes the rank of $H_{\mathcal{A}_{2}}^{3, v-3}(G)$ and the torsion of $H_{\mathcal{A}_{2}}^{4, v-4}(G)$ in terms of the cyclomatic number and subgraphs of $G$. This adds to the description of the first 3 gradings stated in Proposition 6.

\section{Theorem 21.}

$$
\begin{aligned}
& \text { rk } H_{\mathcal{A}_{2}, v-3}^{3, G}= \begin{cases}p_{1}+\left(\begin{array}{c}
p_{1}+1 \\
3
\end{array}\right)-t_{2} & G \text { bipartite } \\
p_{1}+\left(\begin{array}{c}
p_{1}+1 \\
3
\end{array}\right)-t_{1}\left(p_{1}-1\right)-t_{2}+2 t_{3}-1 & \text { otherwise }\end{cases} \\
& \text { tor } H_{\mathcal{A}_{2}}^{4, v-4}(G)= \begin{cases}\mathbb{Z}_{2}^{p_{1}+\left(\begin{array}{c}
p_{1}+1 \\
3
\end{array}\right)-t_{2}} & G \text { bipartite } \\
\mathbb{Z}_{2}^{p_{1}+\left({ }^{p_{1}+1}\right)-t_{1}\left(p_{1}-1\right)-t_{2}+2 t_{3}-1} & \text { otherwise }\end{cases}
\end{aligned}
$$

Proof. Let the chromatic polynomial of $G$ have coefficients labeled as follows:

$$
P_{G}(\lambda)=\lambda^{v}+c_{v-1} \lambda^{v-1}+\ldots+c_{2} \lambda^{2}+c_{1} \lambda
$$

We change variables to $\lambda=q+1$ to match the graded Euler characteristic of $H_{\mathcal{A}_{2}}(G)$. The coefficient 
of $q^{v-i}$ in this polynomial will be denoted $a_{i}$ throughout this section.

$$
\begin{aligned}
P_{G}(q) & =(q+1)^{v}+c_{v-1}(q+1)^{v-1}+\ldots+c_{2}(q+1)^{2}+c_{1}(q+1) \\
& =q^{v}+a_{v-1} q^{v-1}+\ldots+a_{2} q^{2}+a_{1} q+a_{0} .
\end{aligned}
$$

Since chromatic homology is supported on only two diagonals, $a_{v-3}=\operatorname{rk} H_{\mathcal{A}_{2}}^{2, v-3}(G)-\mathrm{rk} H_{\mathcal{A}_{2}}^{3, v-3}(G)$. By [CCR08, Cor. 4.2], rk $H_{\mathcal{A}_{2}}^{2, v-3}(G)=\operatorname{rk} H_{\mathcal{A}_{2}}^{1, v-1}(G)$. The rank of $H_{\mathcal{A}_{2}}^{1, v-1}(G)$ is known (see Proposition 6$)$, so

$$
\operatorname{rk} H_{\mathcal{A}_{2}}^{3, v-3}(G)=\operatorname{rk} H_{\mathcal{A}_{2}}^{2, v-3}(G)-a_{v-3}=\operatorname{rk} H_{\mathcal{A}_{2}}^{1, v-1}(G)-a_{v-3}= \begin{cases}p_{1}-a_{v-3} & G \text { bipartite } \\ p_{1}-1-a_{v-3} & \text { otherwise }\end{cases}
$$

Using formulas in Theorem 20, we compute $a_{v-3}$.

$$
\begin{aligned}
a_{v-3} & =\left(\begin{array}{c}
v \\
v-3
\end{array}\right)+c_{v-1}\left(\begin{array}{l}
v-1 \\
v-3
\end{array}\right)+c_{v-2}\left(\begin{array}{l}
v-2 \\
v-3
\end{array}\right)+c_{v-3} \\
& =\left(\begin{array}{c}
v \\
v-3
\end{array}\right)-E\left(\begin{array}{c}
v-1 \\
2
\end{array}\right)+\left(\left(\begin{array}{c}
E \\
2
\end{array}\right)-t_{1}\right)(v-2)-\left(\begin{array}{c}
E \\
3
\end{array}\right)+(E-2) t_{1}+t_{2}-2 t_{3} \\
& =-\frac{1}{6}(E-v)(1+E-v)(2+E-v)+t_{1}(E-v)+t_{2}-2 t_{3} \\
& =-\left(\begin{array}{c}
p_{1}+1 \\
3
\end{array}\right)+t_{1}\left(p_{1}-1\right)+t_{2}-2 t_{3}
\end{aligned}
$$

Note that $t_{1}=t_{3}=0$ if $G$ is bipartite.

The rank of $H_{\mathcal{A}_{2}}^{4, v-4}(G)$ and the torsion of $H_{\mathcal{A}_{2}}^{5, v-5}(G)$ can be described using a proof similar to that of the previous result.

\section{Theorem 22.}

$$
\begin{array}{r}
\text { rk } H_{\mathcal{A}_{2}}^{4, v-4}(G)=\left\{\begin{array}{lr}
\left(\begin{array}{c}
p_{1} \\
2
\end{array}\right)+a_{v-4} & \text { G bipartite } \\
\left(\begin{array}{c}
p_{1} \\
2
\end{array}\right)-t_{1}+1+a_{v-4} & \text { otherwise }
\end{array}\right. \\
\text { tor } H_{\mathcal{A}_{2}}^{5, v-5}(G)= \begin{cases}\left.\mathbb{Z}_{2}^{\left(p_{1}\right.} 2\right)+a_{v-4} & G \text { bipartite } \\
\left.\mathbb{Z}_{2}^{\left(p_{1}\right.} 2\right)-t_{1}+1+a_{v-4} & \text { otherwise }\end{cases}
\end{array}
$$

The coefficient $a_{v-4}$ depends on $v$ and on the first five coefficients of $P_{G}(\lambda)$.

$$
a_{v-4}=\left(\begin{array}{l}
v \\
4
\end{array}\right)-E\left(\begin{array}{c}
v-1 \\
3
\end{array}\right)+\left(\left(\begin{array}{c}
E \\
2
\end{array}\right)-t_{1}\right)\left(\begin{array}{c}
v-2 \\
2
\end{array}\right)+c_{v-3}(v-3)+c_{v-4} .
$$

Proof. Similar to the proof of Theorem 21, using Theorem 20 and the equation $\operatorname{rk} H_{\mathcal{A}_{2}}^{4, v-4}(G)=$ 
$\operatorname{rk} H_{\mathcal{A}_{2}}^{2, v-2}(G)+a_{v-4}$

Finally, we describe the rank of $H_{\mathcal{A}_{2}}^{5, v-5}(G)$ and the torsion of $H_{\mathcal{A}_{2}}^{6, v-6}(G)$. This approach can theoretically be extended to further groups on the diagonal, using the method of [Bie05] to find formulas for additional chromatic coefficients.

\section{Theorem 23.}

$$
\begin{aligned}
& \operatorname{rk} H_{\mathcal{A}_{2}}^{5, v-5}(G)= \begin{cases}p_{1}+\left(\begin{array}{c}
p_{1}+1 \\
3
\end{array}\right)-t_{2}-a_{v-5} & G \text { bipartite } \\
p_{1}+\left(\begin{array}{c}
p_{1}+1 \\
3
\end{array}\right)-t_{1}\left(p_{1}-1\right)-t_{2}+2 t_{3}-1-a_{v-5} & \text { otherwise }\end{cases} \\
& \text { tor } H_{\mathcal{A}_{2}}^{6, v-6}(G)= \begin{cases}\mathbb{Z}_{2}^{p_{1}+\left(\begin{array}{c}
p_{1}+1 \\
3
\end{array}\right)-t_{2}-a_{v-5}} & G \text { bipartite } \\
\mathbb{Z}_{2}^{p_{1}+\left(\begin{array}{c}
p_{1}+1 \\
3
\end{array}\right)-t_{1}\left(p_{1}-1\right)-t_{2}+2 t_{3}-1-a_{v-5}} & \text { otherwise }\end{cases}
\end{aligned}
$$

The coefficient $a_{v-5}$ depends on $v$ and on the first six coefficients of $P_{G}(\lambda)$.

$$
a_{v-5}=\left(\begin{array}{l}
v \\
5
\end{array}\right)-E\left(\begin{array}{c}
v-1 \\
4
\end{array}\right)+\left(\left(\begin{array}{l}
E \\
2
\end{array}\right)-t_{1}\right)\left(\begin{array}{c}
v-2 \\
3
\end{array}\right)+c_{v-3}\left(\begin{array}{c}
v-3 \\
2
\end{array}\right)+c_{v-4}(v-4)+c_{v-5} .
$$

Proof. Similar to the proof of Theorem 21, using Theorem 20 and the equation $\operatorname{rk} H_{\mathcal{A}_{2}}^{5, v-5}(G)=$ rk $H_{\mathcal{A}_{2}}^{3, v-3}(G)-a_{v-5}$.

For a reduced alternating diagram $D$, the first three coefficients of the Jones polynomial may be stated in terms of the all- $A$-state graph $A(D)$ [DL06] which is equivalent to our all-positive graph $G_{+}(D)$. If $G_{+}(D)$ has girth $\ell$, we can extend this description to the first $\ell$ coefficients of the Jones polynomial via the following result about chromatic coefficients.

Theorem 24. [Mer72] If $G$ is a graph with girth $\ell>2$ and $n_{\ell}$ cycles of length $\ell$, then the first $\ell$ coefficients of the chromatic polynomial are:

$$
c_{v-i}= \begin{cases}(-1)^{i}\left(\begin{array}{c}
E \\
i
\end{array}\right) & 0 \leq i<\ell-1 \\
(-1)^{\ell-1}\left(\left(\begin{array}{c}
E \\
\ell-1
\end{array}\right)-n_{\ell}\right) & i=\ell-1\end{cases}
$$

Remark 3. The statement of this result in [Mer72, Theorem 2] is not explicitly restricted to graphs with $\ell>2$. In the case $i=\ell-1$, the proof contains an assumption that the number of cycle-containing subgraphs with $v-1$ connected components and $t$ edges is zero for $t>2$; this is not true for graphs with edge multiplicities of 3 or higher.

Theorem 25. Let $D$ be a diagram of a link $L$ such that $K h(L)$ is homologically thin and the girth of $G_{+}(D)$ is $\ell>2$. Let $p_{1}$ be the cyclomatic number of $G_{+}(D)$, and $n_{\ell}$ be the number of $\ell$-cycles. 
Then the first $\ell-1$ coefficients of the Jones polynomial $J(L)$ are given by the sequence:

$$
1,-p_{1},\left(\begin{array}{c}
p_{1}+1 \\
2
\end{array}\right), \ldots,(-1)^{i}\left(\begin{array}{c}
p_{1}-1+i \\
i
\end{array}\right), \ldots
$$

and the $\ell$ th coefficient is $(-1)^{\ell-1}\left(\left(\begin{array}{c}p_{1}-1+(\ell-1) \\ \ell-1\end{array}\right)-n_{\ell}\right)$. If we consider the all-negative state graph $G_{-}(D)$, an analogous statement holds for the first $\ell\left(G_{-}(D)\right)$ ultimate coefficients of $J(L)$.

Proof. For notational convenience, let $G=G_{+}(D)$. We first change variables from $\lambda$ to $q+1$ and calculate the coefficients of $P_{G}(q)$, in the same manner as in the proof of Theorem 21 .

$$
\begin{aligned}
P_{G}(q) & =(q+1)^{v}+c_{v-1}(q+1)^{v-1}+\ldots+c_{2}(q+1)^{2}+c_{1}(q+1) \\
& =q^{v}+a_{v-1} q^{v-1}+\ldots+a_{2} q^{2}+a_{1} q+a_{0} .
\end{aligned}
$$

The coefficient on the term $q^{v-i}$ is given by

$$
a_{v-i}=\left(\begin{array}{c}
v \\
v-i
\end{array}\right)+c_{v-1}\left(\begin{array}{c}
v-1 \\
v-i
\end{array}\right)+\ldots+c_{v-i}\left(\begin{array}{c}
v-i \\
v-i
\end{array}\right)=\sum_{k=0}^{i} c_{v-k}\left(\begin{array}{c}
v-k \\
v-i
\end{array}\right)
$$

For $i<\ell-1$, we combine this formula with Theorem 24. (See Appendix A, Proposition 10.)

$$
a_{v-i}=\sum_{k=0}^{i} c_{v-k}\left(\begin{array}{c}
v-k \\
v-i
\end{array}\right)=\sum_{k=0}^{i}(-1)^{k}\left(\begin{array}{l}
E \\
k
\end{array}\right)\left(\begin{array}{c}
v-k \\
v-i
\end{array}\right)=(-1)^{i}\left(\begin{array}{c}
p_{1}-2+i \\
i
\end{array}\right)
$$

For $i=\ell-1$, a similar computation shows that $a_{v-\ell-1}=(-1)^{\ell-1}\left(\left(\begin{array}{c}p_{1}-2+(\ell-1) \\ \ell-1\end{array}\right)-n_{\ell}\right)$. Now let the Jones polynomial of $L$ be written with positive first coefficient as

$$
J_{L}(q)=\beta_{0} q^{C}+\beta_{1} q^{C+2}+\beta_{2} q^{C+4}+\beta_{3} q^{C+6}+\ldots
$$

We write the unnormalized Jones polynomial with coefficients $\alpha_{i}$ :

$$
\begin{aligned}
\hat{J}_{L}(q) & =\left(q+q^{-1}\right) J_{L}(q) \\
& =\left(\beta_{0} q^{C-1}+\beta_{0} q^{C+1}\right)+\left(\beta_{1} q^{C+1}+\beta_{1} q^{C+3}\right)+\left(\beta_{2} q^{C+3}+\beta_{2} q^{C+5}\right)+\left(\beta_{3} q^{C+5}+\beta_{3} q^{C+7}\right)+\ldots \\
& =\beta_{0} q^{C-1}+\left(\beta_{0}+\beta_{1}\right) q^{C+1}+\left(\beta_{1}+\beta_{2}\right) q^{C+3}+\left(\beta_{2}+\beta_{3}\right) q^{C+5}+\ldots \\
& =\alpha_{0} q^{C-1}+\alpha_{1} q^{C+1}+\alpha_{2} q^{C+3}+\alpha_{3} q^{C+5}+\ldots
\end{aligned}
$$

We observe the following relationship: $\alpha_{0}=\beta_{0}=1$ and for $n>0, \alpha_{n}=\beta_{n-1}+\beta_{n}$. Solving for $\beta_{n}$ explicitly in terms of the $\alpha$, we find $\beta_{n}=\sum_{i=0}^{n}(-1)^{n+i} \alpha_{i}$ for $n>0$.

Since $K h(L)$ lies only on two diagonals, the isomorphism of Theorem 7 implies that $\alpha_{i}=a_{v-i}$ 
for $0 \leq i \leq \ell-1$, where the $a_{v-i}$ s are the coefficients of the chromatic polynomial $P_{G}(q)$. Hence we can express the coefficient $\beta_{n}$ in terms of the $a_{v-i}$ s. (See Appendix A, Proposition 11.)

$$
\beta_{n}=\sum_{i=0}^{n}(-1)^{n+i} a_{v-i}=\sum_{i=0}^{n}(-1)^{n}\left(\begin{array}{c}
p_{1}-2+i \\
i
\end{array}\right)=(-1)^{n}\left(\begin{array}{c}
p_{1}-1+n \\
n
\end{array}\right)
$$

for $n<\ell-1$, and

$$
\begin{aligned}
\beta_{\ell-1} & =\sum_{i=0}^{\ell-2}(-1)^{\ell-1+i}(-1)^{i}\left(\begin{array}{c}
p_{1}-2+i \\
i
\end{array}\right)+(-1)^{\ell-1}\left(\left(\begin{array}{c}
p_{1}+2+(\ell-1) \\
\ell-1
\end{array}\right)-n_{\ell}\right) \\
& =(-1)^{\ell-1}\left(\sum_{i=0}^{\ell-2}\left(\begin{array}{c}
p_{1}-2+i \\
i
\end{array}\right)+\left(\begin{array}{c}
p_{1}+2+(\ell-1) \\
\ell-1
\end{array}\right)\right)-(-1)^{\ell-1} n_{\ell} \\
& =(-1)^{\ell-1}\left(\left(\begin{array}{c}
p_{1}-1+(\ell-1) \\
\ell-1
\end{array}\right)-n_{\ell}\right) .
\end{aligned}
$$

Example 3. Let $K=12 a 1242$ (the $(-3,-4,-5)$ pretzel knot) with Jones polynomial $J(K)=$ $q^{-22}-2 q^{-20}+3 q^{-18}-4 q^{-16}+5 q^{-14}-6 q^{-12}+6 q^{-10}-6 q^{-8}+5 q^{-6}-4 q^{-4}+3 q^{-2}-1+q^{2}$. This knot has a diagram whose all-positive state graph is $\left.P_{7}\right|^{3} P_{8}$ with $\ell=7$ and $p_{1}=2$. The first 6 coefficients are equal to $(-1)^{i}\left(\begin{array}{c}1+i \\ i\end{array}\right)=1,-2,3,-4,5,-6$ and the rth coefficient is $(-1)^{6}\left(\begin{array}{l}7 \\ 6\end{array}\right)-1=6$.

Corollary 15. Let $D$ be a diagram of $L$ with $c_{+}$positive crossings and $c_{-}$negative crossings. Let $\ell$ be the girth of $G_{+}(D), p_{1}$ be the cyclomatic number, and $n_{i}$ equal to the number of $i$-cycles. If $3 \leq i<\ell$ with $i$ odd, then

$$
\operatorname{rk} K h^{i-c_{-},-v+2 i+c_{+}-2 c_{-}}(L)=\left(\sum_{k \text { odd }, 3 \leq k \leq i}\left(\begin{array}{c}
p_{1}-2+k \\
k
\end{array}\right)\right)+p_{1}-n_{i+1}-\delta_{\text {odd }}
$$

where $\delta_{\text {odd }}=1$ if $G_{+}(D)$ has an odd cycle and 0 otherwise.

If $3<i<\ell$ with $i$ even, then

$$
\operatorname{rk} K h^{i-c_{-},-v+2 i+c_{+}-2 c_{-}}(L)=\left(\sum_{k \text { even, } 2 \leq k \leq i}\left(\begin{array}{c}
p_{1}-2+k \\
k
\end{array}\right)\right)-n_{i+1}+\delta_{\text {odd }}
$$

where $\delta_{\text {odd }}=1$ if $G_{+}(D)$ has an odd cycle and 0 otherwise.

By Theorems 5 and 7 , these formulas also give the number of $\mathbb{Z}_{2}$-torsion groups on the next grading of this diagonal: tor $K h^{(i+1)-c_{-},-v+2(i+1)+c_{+}-2 c_{-}}(L)$. If we consider the all-negative state graph $G_{-}(D)$, an analogous statement holds for the highest homological gradings in $K h(L)$.

Proof. We prove the statement for $i=3$ and $i=4$ and use induction on these base cases. Note 
that the general formula for a coefficient of the Jones polynomial of a homologically thin link is $\alpha_{k}=(-1)^{k-c_{-}} \mathrm{rk} K h^{k-c_{-},-v+2 k+c_{+}-2 c_{-}}(L)+(-1)^{(k-1)-c_{-}} \mathrm{rk} K h^{(k-1)-c_{-},-v+2 k+c_{+}-2 c_{-}}(L)$. In our convention for the Jones polynomial, we fix the sign of the leading term to be positive, and the other coefficients alternate in sign. Thus, in this context, we will write

$$
\alpha_{k}=(-1)^{k} \mathrm{rk} K h^{k-c_{-},-v+2 k+c_{+}-2 c_{-}}(L)+(-1)^{k-1} \mathrm{rk} K h^{(k-1)-c_{-},-v+2 k+c_{+}-2 c_{-}}(L) .
$$

Suppose $i=3<\ell$ :

$$
\begin{aligned}
\alpha_{3}=a_{v-3} & =-\mathrm{rk} K h^{3-c_{-},-v+6+c_{+}-2 c_{-}}(L)+\mathrm{rk} K h^{2-c_{-},-v+6+c_{+}-2 c_{-}}(L) \\
& =-\mathrm{rk} K h^{3-c_{-},-v+6+c_{+}-2 c_{-}}(L)+\mathrm{rk} K h^{1-c_{-},-v+2+c_{+}-2 c_{-}}(L) \\
& =-\mathrm{rk} K h^{3-c_{-},-v+6+c_{+}-2 c_{-}}(L)+ \begin{cases}p_{1} & G \text { bipartite } \\
p_{1}-1 & G \text { otherwise }\end{cases}
\end{aligned}
$$

The girth of $G$ may be 4 or greater, so from Theorem 24 and its proof we see that $a_{v-3}=$ $-\left(\begin{array}{c}p_{1}+1 \\ 3\end{array}\right)+n_{4}$. The formula for $\mathrm{rk} K h^{3-c_{-},-v+6+c_{+}-2 c_{-}}(L)$ follows from the equation above.

Now suppose $i=4<\ell$ :

$$
\begin{aligned}
\alpha_{4}=a_{v-4} & =\operatorname{rk} K h^{4-c_{-},-v+8+c_{+}-2 c_{-}}(L)-\operatorname{rk} K h^{3-c_{-},-v+8+c_{+}-2 c_{-}}(L) \\
& =\operatorname{rk} K h^{4-c_{-},-v+8+c_{+}-2 c_{-}}(L)-\operatorname{rk} K h^{2-c_{-},-v+4+c_{+}-2 c_{-}}(L) \\
& =\operatorname{rk} K h^{4-c_{-},-v+8+c_{+}-2 c_{-}}(L)+\left\{\begin{array}{cc}
\left(\begin{array}{c}
p_{1} \\
2
\end{array}\right) & G \text { bipartite } \\
\left(\begin{array}{c}
p_{1} \\
2
\end{array}\right)+1 & G \text { otherwise }
\end{array}\right.
\end{aligned}
$$

The girth of $G$ may be 5 or greater, so $a_{v-4}=\left(\begin{array}{c}p_{1}+2 \\ 4\end{array}\right)-n_{5}$. Then we use the equation above to determine the formula for $\mathrm{rk} K h^{4-c_{-},-v+8+c_{+}-2 c_{-}}(L)$.

For the induction step, let $3<i<\ell$ with $i$ odd. Then

$$
\begin{aligned}
& \alpha_{i}=a_{v-i}=-\mathrm{rk} K h^{i-c_{-},-v+2 i+c_{+}-2 c_{-}}(L)+\mathrm{rk} K h^{(i-1)-c_{-},-v+2 i+c_{+}-2 c_{-}}(L) \\
& =-\mathrm{rk} K h^{i-c_{-},-v+2 i+c_{+}-2 c_{-}}(L)+\mathrm{rk} K h^{(i-2)-c_{-},-v+2(i-2)+c_{+}-2 c_{-}}(L) \\
& =-\mathrm{rk} K h^{i-c_{-},-v+2 i+c_{+}-2 c_{-}}(L)+\left(\sum_{k \text { odd }, 3 \leq k \leq i-2}\left(\begin{array}{c}
p_{1}-2+k \\
k
\end{array}\right)\right)+p_{1}-\delta_{\text {odd }}
\end{aligned}
$$

Note the absence of an $n_{i-1}$ term in the expression for rk $K h^{(i-2)-c_{-},-v+2(i-2)+c_{+}-2 c_{-}}(L)$, since we know $\ell>i-1$. 
Since $a_{v-i}=-\left(\begin{array}{c}p_{1}-2+i \\ i\end{array}\right)+n_{i+1}$, we have:

$$
\begin{aligned}
\operatorname{rk} & K h^{i-c_{-},-v+2 i+c_{+}-2 c_{-}(L)} \\
& =\left(\sum_{k \text { odd, } 3 \leq k \leq i-2}\left(\begin{array}{c}
p_{1}-2+k \\
k
\end{array}\right)\right)+p_{1}-\delta_{\text {odd }}+\left(\begin{array}{c}
p_{1}-2+i \\
i
\end{array}\right)-n_{i+1} \\
& =\left(\sum_{k \text { odd }, 3 \leq k \leq i}\left(\begin{array}{c}
p_{1}-2+k \\
k
\end{array}\right)\right)+p_{1}-n_{i+1}-\delta_{\text {odd }} .
\end{aligned}
$$

Finally, let $4<i<\ell$ with $i$ even. Then

$$
\begin{aligned}
& \alpha_{i}=a_{v-i}=-\mathrm{rk} K h^{i-c_{-},-v+2 i+c_{+}-2 c_{-}}(L)+\mathrm{rk} K h^{(i-1)-c_{-},-v+2 i+c_{+}-2 c_{-}}(L) \\
& =-\mathrm{rk} K h^{i-c_{-},-v+2 i+c_{+}-2 c_{-}}(L)+\mathrm{rk} K h^{(i-2)-_{-},-v+2(i-2)+c_{+}-2 c_{-}}(L) \\
& =-\mathrm{rk} K h^{i-c_{-},-v+2 i+c_{+}-2 c_{-}}(L)+\left(\sum_{k \text { even, } 4 \leq k \leq i-2}\left(\begin{array}{c}
p_{1}-2+k \\
k
\end{array}\right)\right)+\delta_{\text {odd }}
\end{aligned}
$$

Since $a_{v-i}=\left(\begin{array}{c}p_{1}-2+i \\ i\end{array}\right)-n_{i+1}$, we have:

$$
\begin{aligned}
\operatorname{rk} K h^{i-c_{-},-v+2 i+c_{+}-2 c_{-}}(L) & =\left(\sum_{k \text { even, } 4 \leq k \leq i-2}\left(\begin{array}{c}
p_{1}-2+k \\
k
\end{array}\right)\right)+\delta_{\text {odd }}+\left(\begin{array}{c}
p_{1}-2+i \\
i
\end{array}\right)-n_{i+1} \\
& =\left(\sum_{k \text { even, } 4 \leq k \leq i}\left(\begin{array}{c}
p_{1}-2+k \\
k
\end{array}\right)\right)-n_{i+1}+\delta_{\text {odd }} .
\end{aligned}
$$

Future work will focus on extending Theorem 25 and Corollary 15 to large classes of links whose Khovanov homology is thick. In Chapter 5, we apply Theorem 25 to find upper bounds for possible girths of $G_{+}(D)$ over all diagrams $D$ of $L$. 


\section{Chapter 4}

\section{Structure of chromatic homology over $\mathcal{A}_{m}=\mathbb{Z}[x] /\left(x^{m}\right), m>2$}

In this section we provide generalizations of some of the results and patterns observed in chromatic homology over $\mathcal{A}_{2}$ to the algebra $\mathcal{A}_{m}=\mathbb{Z}[x] /\left(x^{m}\right)$, focusing on $m=3$. We show that some properties which are constant over $\mathcal{A}_{2}$, such as width, become dependent both on the choice of algebra $\mathcal{A}_{m}$ for $m>2$, and and the choice of graph. These preliminary results indicate that chromatic homology may have richer algebraic structure over other algebras and may be better at distinguishing graphs.

\subsection{Width of chromatic homology over $\mathcal{A}_{m}$}

Computations indicate that the homological span of chromatic homology is invariant under the choice of algebra $\mathcal{A}_{m}$.

Conjecture 1. The homological span of chromatic homology over algebra $\mathcal{A}_{m}$ of any graph $G$ with $v$ vertices and b blocks is equal to $\operatorname{hspan}\left(H_{\mathcal{A}_{m}}(G)\right)=v-b$.

At the moment, we can only show that we have a lower bound on width following the reasoning in Theorem 8 and basic results from [HGPR06]:

Theorem 26. Homological span of chromatic homology over any algebra $\mathcal{A}_{m}$ depends only on the number of vertices $v$ and blocks $b$ of a graph $G$ : hspan $\left(H_{\mathcal{A}_{m}}(G)\right) \geq v-b$.

Note that, unlike the case of $\mathcal{A}_{2}$ where width is equal to two, the width of the chromatic homology increases with $m$ and depends on the number of vertices of the graph.

Theorem 27. For any graph $G$ the width of $H_{\mathcal{A}_{m}}(G)$ is equal to hw $\left(H_{\mathcal{A}_{m}}(G)\right)=(m-2) v+2$. 
Proof. In case that $G$ is a tree note that $|E(G)|=v-1$. Next note that $H_{\mathcal{A}_{m}}^{0}(G)=\mathcal{A}_{m} \otimes \mathcal{A}_{m}^{\prime \otimes^{v-1}}$ where $\mathcal{A}_{m}^{\prime}$ is the submodule of $\mathcal{A}_{m}$ such that $\mathcal{A}_{m}=\mathbb{Z}_{\mathbf{1}} \oplus \mathcal{A}^{\prime}$ with $\mathbf{1}$ the identity of $\mathcal{A}_{m}$ (Corollary 2 ). Therefore the highest non-zero homology group is $H_{\mathcal{A}_{m}}^{0,(m-1) v}(G)=\mathbb{Z}$, on the diagonal $i+$ $j=(m-1) v$. The lowest non-zero group in $\mathcal{A}_{m}$ is located on the diagonal $i+j=v-1$, so $\mathrm{hw}\left(H_{\mathcal{A}_{m}}(G)\right)=(m-1) v-(v-1)+1=(m-2) v+2$.

If $G$ is not a tree we still have $H_{\mathcal{A}_{m}}^{0,(m-1) v}(G)=\mathbb{Z}$. It remains to show that there exists a nontrivial entry on $i+j=v-1$ diagonal; i.e., that there exists $j>0$ such that $H_{\mathcal{A}_{m}}^{v-1-j, j}(G) \neq 0$. Arguments in the proof of Theorem 8 generalize to $\mathcal{A}_{m}$ to show that $H_{\mathcal{A}_{m}}^{v-b-1, b}(G)$ is non-trivial, which is precisely the group we needed.

Considering homological span of torsion is somewhat more involved. Note that Hochschild homology implies the following about the span of torsion for cycle graphs:

Proposition 8. For $m>2, H_{\mathcal{A}_{m}}\left(P_{n}\right)$ has one $\mathbb{Z}_{m}$ torsion group on each of $\left\lceil\frac{n}{2}-1\right\rceil$ diagonals.

Proposition 9. The torsion width of chromatic homology of a cycle is given by

$$
\operatorname{hw}^{t}\left(H_{\mathcal{A}_{m}}\left(P_{n}\right)\right)= \begin{cases}\frac{m n}{2}-2 m-n+5, & n \text { even } \\ \frac{m n}{2}-\frac{3}{2} m-n+4, & n \text { odd }\end{cases}
$$

We conjecture that the width of torsion over $\mathcal{A}_{3}$ of any graph depends only on the number of vertices and the girth of the graph.

Conjecture 2. Let $G$ be a simple, connected graph with $v$ vertices and girth $\ell$, with $\ell=2 k$ or $\ell=2 k-1$ depending on parity. Then

$$
\operatorname{hw}^{t}\left(H_{\mathcal{A}_{3}}(G)\right)=\operatorname{hw}^{t}\left(H_{\mathcal{A}_{3}}\left(P_{\ell}\right)\right)+v-\ell=(k-1)+v-\ell= \begin{cases}v-k-1, & \ell \text { even } \\ v-k, & \ell \text { odd }\end{cases}
$$

\section{2 $\quad H^{i_{\max }}(G)$ tail of homology}

The fact that chromatic homology $H_{\mathcal{A}_{2}}(G)$ is supported on two diagonals, has the knight move structure [CCR08], contains no torsion other than $\mathbb{Z}_{2}$ and is completely determined by the chromatic polynomial [LS17] enables us to describe the homology in the maximal homological grading $i_{\max }$. $H_{\mathcal{A}_{2}}^{i_{\max }}(G)$ contains a free group on the lowest diagonal, and since $H_{\mathcal{A}_{2}}^{v-b-1, b}(G)=\mathbb{Z}^{k}$ is the only group in $j_{\text {min }}, k$ is equal to the absolute value of the coefficient on the lowest degree term in $P_{G}(1+q)$. The only other non-trivial group in maximal homological grading $i_{\max }=v-b-1$ is $H_{\mathcal{A}_{2}}^{v-b-1, b+1}(G)$ and it contains a copy of $\mathbb{Z}_{2}$ for every copy of $\mathbb{Z}$ in $H_{\mathcal{A}_{2}}^{v-1, b}(G)$. In the rest of the 
Section we will refer to $H_{\mathcal{A}_{2}}^{i_{\max }}(G)=H_{\mathcal{A}_{2}}^{v-b-1}(G)$ as the tail of chromatic homology of $G$ and denote it as $T l_{\mathcal{A}_{2}}(G)$. Notice that the tail of a cycle $P_{n}$ is $T l_{\mathcal{A}_{2}}\left(P_{n}\right)=$\begin{tabular}{|l|}
$\mathbb{Z}_{2}$ \\
\hline $\mathbb{Z}$
\end{tabular}

The tail of any graph consists of some number of copies of $T l_{2}:=T l_{\mathcal{A}_{2}}\left(P_{n}\right)$. The rest of the section contains explicit computations of the tail of chromatic homology based on knowing the lowest coefficient of $P_{G}(1+q)$.

Theorem 28. If $G$ is a connected outerplanar graph, then $T l_{\mathcal{A}_{2}}(G)=T l_{2}$.

Proof. If $G$ has $r_{k} k$-gons and $b$ blocks, then $P_{G}(\lambda)=(-1)^{n} \lambda(\lambda-1)^{b} \prod_{k \geq 3}(1+(1-\lambda)+(1-$ $\left.\lambda)^{2}+\ldots+(1-\lambda)^{k-2}\right)^{r_{k}}$ where $n=\sum_{k \geq 3} r_{k}(k-2)([$ WW92, Theorem 2]). Under the variable change $\lambda=1+q$, the chromatic polynomial of $G$ becomes the $q$-polynomial

$$
(-1)^{n}(1+q) q^{b} \prod_{k \geq 3}\left(1+(-q)+(-q)^{2}+\ldots+(-q)^{k-2}\right)^{r_{k}}
$$

The lowest degree term in this polynomial has coefficient \pm 1 so we get only one copy of $T l_{2}$ in the tail of $G$.

Definition 22. A chord is an edge that joins two vertices of $P_{n}$ but is not itself an edge of $P_{n}$. A chordal graph is a graph in which every cycle of length 4 or higher has a chord. In other words, chordal graphs contain no induced cycles of length greater than 3.

Theorem 29. If $G$ is a chordal graph, $T l_{\mathcal{A}_{2}}(G)$ is the direct sum of $2^{s_{3}} 3^{s_{4}} \cdots(k-1)^{s_{k}}$ copies of $T l_{2}$, where $s_{k}$ is the exponent of $(\lambda-k)$ in $P_{G}(\lambda)$.

Proof. If $G$ is a chordal graph with $v$ vertices, then $P_{G}(\lambda)=\lambda^{s_{0}}(\lambda-1)^{s_{1}}(\lambda-2)^{s_{2}} \cdots(\lambda-k)^{s_{k}}$ with $s_{i} \geq 0, \forall i$ such that $\sum_{i=0}^{k} s_{i}=v\left(\left[\right.\right.$ DKT05]). Next $P_{G}(1+q)=(1+q)^{s_{0}}(q)^{s_{1}}(-1+q)^{s_{2}} \cdots(-(k-$ $1)+q)^{s_{k}}$ whose lowest degree term is $(-1)^{S} 2^{s_{3}} 3^{s_{4}} \cdots(k-1)^{s_{k}} q^{s_{1}}$, where $S=\sum_{i=1}^{k} s_{i}$. The absolute value of the coefficient of the lowest degree term is $2^{s_{3}} 3^{s_{4}} \cdots(k-1)^{s_{k}}$.

Corollary 16. Let $K_{n}$ denote the complete graph on $n$ vertices and $W_{n}$ the wheel graph. Then $T l_{\mathcal{A}_{2}}\left(K_{n}\right) \cong T l_{2}^{\oplus(n-2) !}$, and $T l_{\mathcal{A}_{2}}\left(W_{n}\right)=T l_{2}^{\oplus(n-2)}$.

Proof. We use the formulas $P_{K_{n}}(q)=(q+1) q(q-1) \cdots(q-(n-2))$ [DKT05, Example 1.2.2] and $P_{W_{n}}(q)=(q+1)\left((q-1)^{n-1}+(-1)^{n-1}(q-1)\right)$ [DKT05, Cor. 1.5.1]. For the second formula, note that the constant term of the second factor is always zero, while the $q$ term will be $((n-1)-1) q=$ $(n-2) q$ if $n$ is even, and $(-(n-1)+1) q=-(n-2) q$ if $n$ is even.

Conjecture 3. Let $W_{n}^{\text {in }}$ be the graph obtained from $W_{n}$ by removing an edge that connects the central vertex to one of the outer vertices. Then the tail of $T l_{\mathcal{A}_{2}}\left(W_{n}^{i n}\right)=T l_{2}^{\oplus(n-3)}$. 
It is natural to ask if this phenomenon extends to chromatic homology over other algebras. From the Hochschild homology of $\mathcal{A}_{m}$ (see Theorem 6), we know that the tail of $H_{\mathcal{A}_{m}}\left(P_{n}\right)$, denoted by $T l_{m}:=H_{\mathcal{A}_{m}}^{n-2}\left(P_{n}\right)$, consists of $m-1$ copies of $\mathbb{Z}$ with a $\mathbb{Z}_{m}$ in the highest quantum grading.

We conjecture that the tail of any graph $T l_{\mathcal{A}_{m}}(G)$ consist of some number of copies of the tail of a cycle, but proving this statement would require structure theorems such as those existing in the $\mathcal{A}_{2}$ case. For example, computations for small values of $n, m$ hint that Corollary 16 extends to other $\mathcal{A}_{m}$ in the case of complete graphs.

Conjecture 4. The tail of the complete graph $K_{n}$ in chromatic homology over $\mathcal{A}_{m}$ consists of $(n-2)$ ! copies of the tail of $P_{n}$, i.e. $T l_{\mathcal{A}_{m}}\left(K_{n}\right)=T l_{m}^{\oplus(n-2) !}$ for $m \geq 3$.

Conjecture 5. If $G$ is a polygon tree (see Section 3.2.2) with $P$ pendant edges attached, then

$$
T l_{\mathcal{A}_{m}}(G)=\bigoplus_{i=1}^{(m-2) P+1}\left(T l_{m}^{\oplus D_{m, P}^{i}}\{i+(P-1)\}\right)
$$

where $D_{m, P}^{i}$ is the ith coefficient of the multinomial $\left(1+x+\ldots+x^{m-2}\right)^{P}$. In particular, $T l_{\mathcal{A}_{m}}(G)=$ $T l_{m}$ if $G$ is a polygon-tree with no pendant edges.

Example 4. Let $G$ be the graph consisting of a triangle with 2 pendant edges. We will examine the last non-trivial homological grading of $H_{\mathcal{A}_{5}}(G)$. The numbers $D_{5,2}^{i}$ in the formula above come from the multinomial $\left(1+x+x^{2}+x^{3}\right)^{2}=1+2 x+3 x^{2}+4 x^{3}+3 x^{4}+2 x^{5}+x^{6}$ and the tail of $P_{n}$ over $\mathcal{A}_{5}$ is $T l_{5}=\mathbb{Z}\{1\} \oplus \mathbb{Z}\{2\} \oplus \mathbb{Z}\{3\} \oplus \mathbb{Z}\{4\} \oplus \mathbb{Z}_{5}\{5\}$. According to the formula in Conjecture 5 :

$$
\begin{aligned}
T l_{\mathcal{A}_{5}}(G) & =\bigoplus_{i=1}^{7}\left(T l_{5}^{\oplus D_{5,2}^{i}}\{i+1\}\right) \\
& =T l_{5}\{2\} \oplus T l_{5}^{\oplus 2}\{3\} \oplus T l_{5}^{\oplus 3}\{4\} \oplus T l_{5}^{\oplus 4}\{5\} \oplus T l_{5}^{\oplus 3}\{6\} \oplus T l_{5}^{\oplus 2}\{7\} \oplus T l_{5}\{8\} .
\end{aligned}
$$

This agrees with our computation for the tail of $G$ over $\mathcal{A}_{5}$, shown in Table 4.1.

Table 4.1: Computation of $H_{\mathcal{A}_{5}}^{1, j}(G)$ in Mathematica [Sco], where $G$ is a triangle with 2 pendant edges (see Example 4).

\begin{tabular}{|c|c|c|c|c|c|c|c|c|c|c|c|}
\hline$i$ & 13 & 12 & 11 & 10 & 9 & 8 & 7 & 6 & 5 & 4 & 3 \\
\hline 1 & $\mathbb{Z}_{5}$ & $\mathbb{Z} \oplus \mathbb{Z}_{5}^{2}$ & $\mathbb{Z}^{3} \oplus \mathbb{Z}_{5}^{3}$ & $\mathbb{Z}^{6} \oplus \mathbb{Z}_{5}^{4}$ & $\mathbb{Z}^{10} \oplus \mathbb{Z}_{5}^{3}$ & $\mathbb{Z}^{12} \oplus \mathbb{Z}_{5}^{2}$ & $\mathbb{Z}^{12} \oplus \mathbb{Z}_{5}$ & $\mathbb{Z}^{10}$ & $\mathbb{Z}^{6}$ & $\mathbb{Z}^{3}$ & $\mathbb{Z}$ \\
\hline
\end{tabular}




\subsection{Relative strengths of chromatic homology and graph polyno- mials}

As stated in Section 2.3, chromatic homology over $\mathcal{A}_{2}$ is completely determined by the chromatic polynomial. However, this is not necessarily true over other algebras. In this section, we compare $H_{\mathcal{A}_{m}}(G)$ to several well-known graph invariants: the chromatic polynomial, the Tutte polynomial, and the cycle matroid.

Definition 23. [Tut54, Bol98] The Tutte polynomial of graph $G=(V, E)$ is defined by

$$
T_{G}(x, y)=\sum_{A \subseteq E}(x-1)^{r(E)-r(A)}(y-1)^{|A|-r(A)}
$$

where $r(A)=v-k(A)$ and $k(A)$ is the number of components in the spanning subgraph $(V, A)$.

Equivalently, the Tutte polynomial may be defined as the unique function on graphs which satisfies the following three properties:

1. If $G$ has b bridges, $l$ loops, and no other edges, then $T_{G}(x, y)=x^{b} y^{l}$.

2. If $G$ is obtained from graph $H$ by adding $b$ bridges and $l$ loops, and no other edges, then $T_{G}(x, y)=x^{b} y^{l} T_{H}(x, y)$.

3. A deletion-contraction rule holds for any edge $e$ in $G$ which is neither a bridge nor a loop: $T_{G}=T_{G-e}+T_{G / e}$.

The chromatic polynomial of $G$ is the specialization of the Tutte polynomial given by $P_{G}(\lambda)=$ $(-1)^{v-k(G)} \lambda^{k(G)} T_{G}(1-\lambda, 0)$. The Jones polynomial is also a specialization of the Tutte polynomial. Given an alternating diagram of knot $K$ whose Tait graph is $G$, the Jones polynomial of $K$ is the Tutte polynomial of $G$ evaluated on the hyperbola $x y=1$ [Bol98]. The Tutte polynomial is determined by a stronger graph invariant: the cycle matroid (also known as the graphic matroid).

Definition 24. [Whi35, dMN05] $A$ matroid $M$ is an ordered pair $(\mathcal{S}, \mathcal{I})$ consisting of a finite set $\mathcal{S}$ (called the ground set) and a collection $\mathcal{I}$ of subsets of $\mathcal{S}$ (called the independent sets) satisfying the following conditions:

1. $\emptyset \in \mathcal{I}$

2. If $I \in \mathcal{I}$ and $J \subseteq I$, then $J \in \mathcal{I}$.

3. If $I, J \in \mathcal{I}$ and $|J|<|I|$, then there is an element $x$ of $I-J$ such that $J \cup x \in \mathcal{I}$.

The rank-size generating function of $M$ is defined as $F(M ; x, y)=\sum_{A \subseteq S} x^{r(A)} y^{|A|}$ where $r(A)$ is the rank of $A$ (the size of the largest independent set contained in $A$ ). 
The cycle matroid of graph $G=(V, E)$, denoted $M(G)$, is a matroid whose ground set is $E$ and whose linearly independent sets are the acyclic subsets of $E$. The circuits of $M(G)$ (minimal dependent sets) are precisely the edge sets of cycles in $G$. Up to a change of variables, the Tutte polynomial $T_{G}(x, y)$ is equivalent to the generating function $F(M(G) ; x, y)$ [dMN05].

Definition 25. Graphs $G$ and $H$ are 2-isomorphic if $G$ can be transformed into $H$ by means of these two moves:

(1) Gluing or separating components of a graph at a single vertex;

(2) Whitney flip: if $G$ consists of two subgraphs $A$ and $B$ glued at two vertices $\left(v_{A}=v_{B}\right.$ and $\left.w_{A}=w_{B}\right)$, then flip $B$ and reglue with the original vertex identification reversed $\left(v_{A}=w_{B}\right.$ and $\left.w_{A}=v_{B}\right)$. See Figure 4.1.
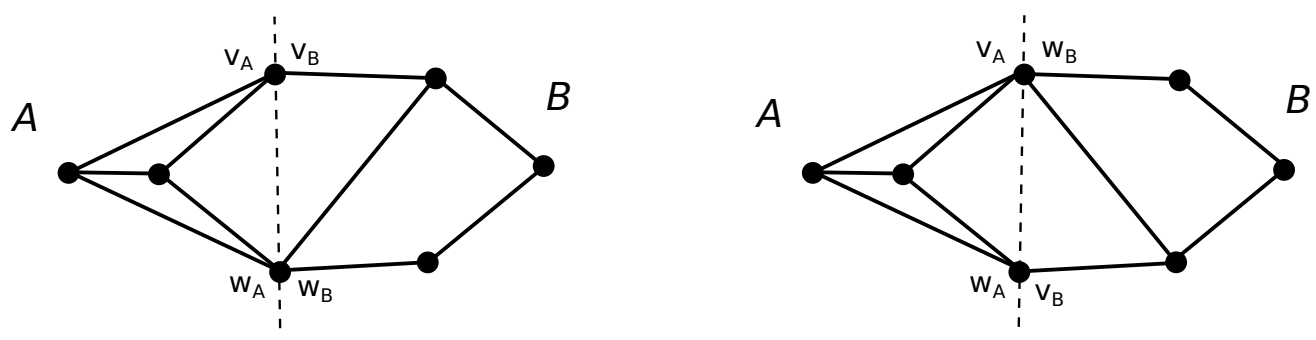

Figure 4.1: Whitney flip operation on graphs.

Theorem 30. [Oxl92, Thm. 5.3.1] $M(G)$ and $M(H)$ are isomorphic matroids if and only if $G$ and $H$ are 2-isomorphic graphs.

Graphs with the same chromatic polynomial may be distinguished by their chromatic homology over $\mathcal{A}_{3}$. For example, the two graphs in Figure 4.2 are cochromatic and share a Tutte polynomial, but $H_{\mathcal{A}_{3}}^{1,9}(A)$ contains an extra copy of $\mathbb{Z}$ which is not found in $H_{\mathcal{A}_{3}}^{1,9}(B)$. The difference which appears in this example can be explained in terms of the edge attachments in the two graphs. In graph $A$, the top triangle shares an edge with another 3 -cycle, while in graph $B$, it has been attached along a different edge which does not have this property. The difference of $\mathbb{Z}$ in chromatic homology is quantified by [PPS09, Proposition 6.3].

In this section we list several examples of cochromatic graphs which are distinguished by chromatic homology over $\mathcal{A}_{3}$. None these pairs differ by an edge attachment and thus are not explained by the result from [PPS09].

Example 5. The graphs in Figure 4.3 appear in [BM76, Exercise 8.4.1] and share the following chromatic polynomial: $\lambda^{6}-10 \lambda^{5}+41 \lambda^{4}-84 \lambda^{3}+84 \lambda^{2}-32 \lambda$. However, $H_{\mathcal{A}_{3}^{1,9}}(A)=\mathbb{Z}^{7} \oplus \mathbb{Z}_{3}^{3}$, which differs from $H_{\mathcal{A}_{3}}^{1,9}(B)=\mathbb{Z}^{8} \oplus \mathbb{Z}_{3}^{3}$. 


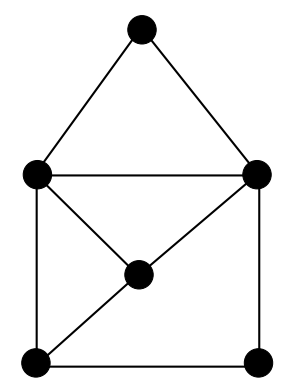

A

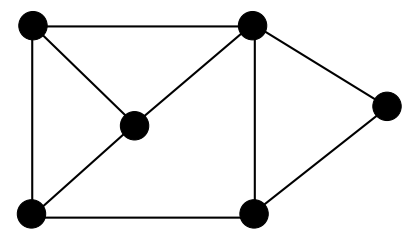

B

Figure 4.2: Cochromatic graphs of Marion C. Gray [PPS09, Example 6.4].

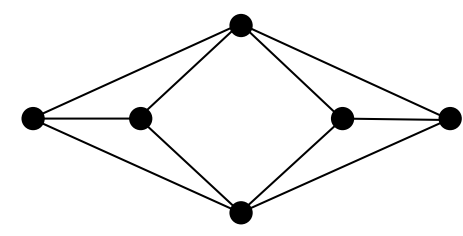

A

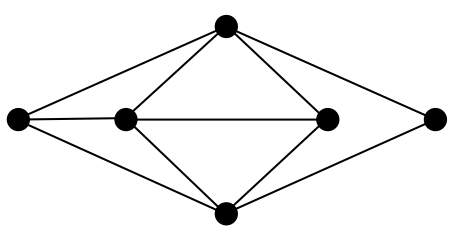

B

Figure 4.3: An example of cochromatic graphs from [BM76].

Example 6. Cochromatic graphs in Figure 4.4 from [CWJ79] and have the following chromatic polynomial: $\lambda^{6}-10 \lambda^{5}+40 \lambda^{4}-80 \lambda^{3}+79 \lambda^{2}-30 \lambda$. Their first chromatic cohomology differ in quantum degree 9: $H_{\mathcal{A}_{3}}^{1,9}(A)=\mathbb{Z}^{5} \oplus \mathbb{Z}_{2} \oplus \mathbb{Z}_{3}^{5}, H_{\mathcal{A}_{3}}^{1,9}(B)=\mathbb{Z}^{6} \oplus \mathbb{Z}_{2} \oplus \mathbb{Z}_{3}^{4}$.

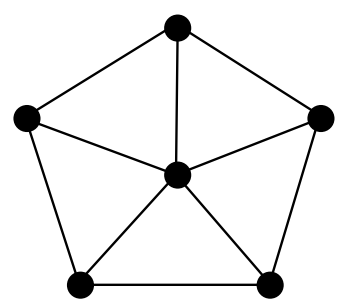

A

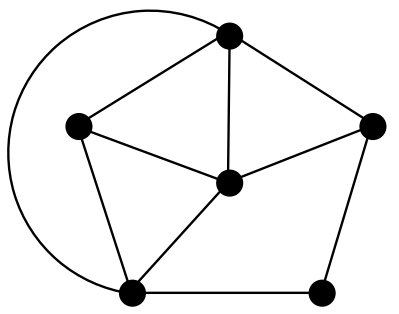

B

Figure 4.4: First example of cochromatic graphs from [CWJ79].

Example 7. The graphs in Figure 4.5, also found in [CWJ79], share the following chromatic polynomial:

$$
\lambda^{7}-11 \lambda^{6}+51 \lambda^{5}-128 \lambda^{4}+184 \lambda^{3}-143 \lambda^{2}+46 \lambda
$$

but $H_{\mathcal{A}_{3}}^{1,11}(A)=\mathbb{Z}^{4} \oplus \mathbb{Z}_{3}^{4}$, which differs from $H_{\mathcal{A}_{3}}^{1,11}(B)=\mathbb{Z}^{5} \oplus \mathbb{Z}_{3}^{3}$. 


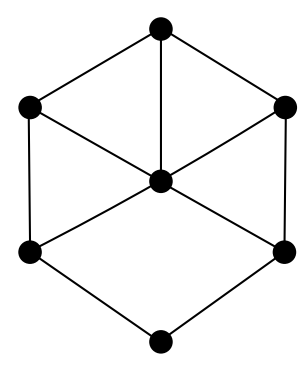

A

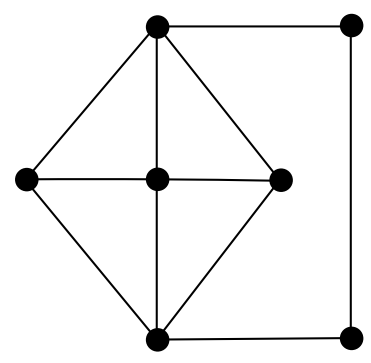

B

Figure 4.5: Second example of cochromatic graphs from [CWJ79].

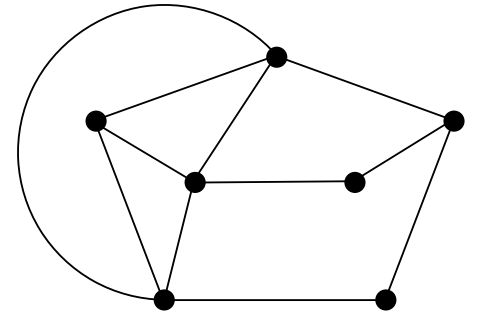

A

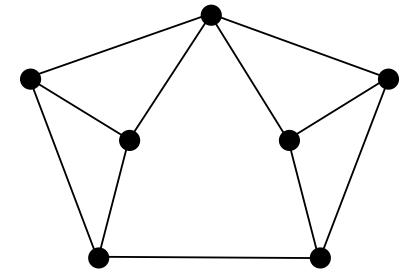

B

Figure 4.6: An example of cochromatic graphs from [KG90].

Example 8. The graphs in Figure 4.6 appear in [KG90] and share the following chromatic polynomial:

$$
\lambda^{7}-11 \lambda^{6}+51 \lambda^{5}-129 \lambda^{4}+188 \lambda^{3}-148 \lambda^{2}+48 \lambda
$$

but $H_{\mathcal{A}_{3}}^{1,11}(A)=\mathbb{Z}^{4} \oplus \mathbb{Z}_{2} \oplus \mathbb{Z}_{3}^{3}$, which differs from $H_{\mathcal{A}_{3}}^{1,11}(B)=\mathbb{Z}^{2} \oplus \mathbb{Z}_{3}^{4}$.

Example 9. The graphs in Figure 4.7 appeared in [DKT05] (attributed to unpublished work by Chee and Royle). They share the following chromatic polynomial:

$$
\lambda^{6}-11 \lambda^{5}+48 \lambda^{4}-103 \lambda^{3}+107 \lambda^{2}-42 \lambda
$$

but $H_{\mathcal{A}_{3}}^{1,9}(A) \cong \mathbb{Z}^{10} \oplus \mathbb{Z}_{2} \oplus \mathbb{Z}_{3}^{4}$, which differs from $H_{\mathcal{A}_{3}}^{1,9}(B) \cong \mathbb{Z}^{9} \oplus \mathbb{Z}_{2} \oplus \mathbb{Z}_{3}^{5}$. Note that [DKT05, Example 3.2.3] has additional examples of cochromatic graphs for which the computation of $H_{\mathcal{A}_{3}}$ exceeds our current resources.

The bigrading $H_{\mathcal{A}_{3}}^{1,2 v-3}(G)$ featured in the above examples is a cycle matroid invariant [PPS09, Theorem 6.2]. The following example demonstrates that the next quantum grading over $\mathcal{A}_{3}, j=$ $2 v-4$, can distinguish 2-isomorphic graphs. 


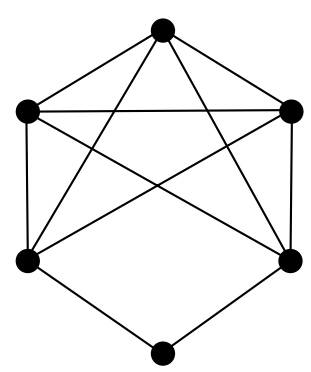

A

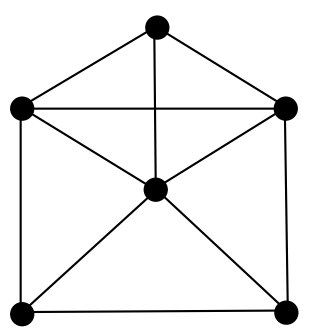

B

Figure 4.7: An example of cochromatic graphs from [DKT05].

Example 10. The graphs in Figure 4.8 are related via a Whitney twist on vertices $v$ and $w$. Therefore they are 2-isomorphic and have the same Tutte polynomial

$T(x, y)=x+3 x^{2}+4 x^{3}+4 x^{4}+3 x^{5}+x^{6}+y+4 x y+5 x^{2} y+4 x^{3} y+2 x^{4} y+2 y^{2}+3 x y^{2}+x^{2} y^{2}+y^{3}$.

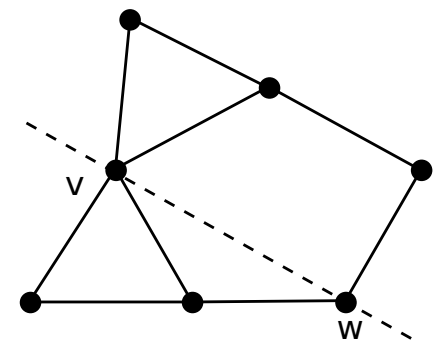

$\mathrm{G}_{1}$

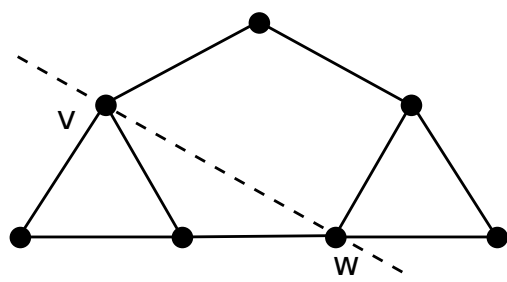

$\mathrm{G}_{2}$

Figure 4.8: Two graphs in the same 2-isomorphism class.

However their chromatic homology over $\mathcal{A}_{3}$ differs already in the zeroth homology group:
$H_{\mathcal{A}_{3}}^{0,10}\left(G_{1}\right)=\mathbb{Z}^{11}$
$H_{\mathcal{A}_{3}}^{1,10}\left(G_{1}\right)=\mathbb{Z}^{5} \oplus \mathbb{Z}_{3}^{8}$
$H_{\mathcal{A}_{3}}^{0,10}\left(G_{2}\right)=\mathbb{Z}^{10}$
$H_{\mathcal{A}_{3}}^{1,10}\left(G_{2}\right)=\mathbb{Z}^{4} \oplus \mathbb{Z}_{3}^{9}$ 


\section{Chapter 5}

\section{The girth of a link}

Each planar diagram of a link $L$ has an associated state graph, whose chromatic homology is related to Khovanov homology by the correspondence described in Theorem 7 . The extent of the correspondence depends on the girth of the associated graph, which varies widely over the set of possible diagrams. For example, adding a right-hand twist to any strand of a diagram $D$ adds a loop to the corresponding all-positive state graph, reducing the girth to 1 . In order to determine the largest correspondence between the Khovanov homology of a link and the chromatic homology of a graph, we would like to identify a diagram which yields a graph of maximal girth. This motivates the definition of a new link invariant $\operatorname{gr}(L)$, the maximum girth over all diagrams, and describe properties of this invariant for certain knots and links. We use the coefficients of the Jones polynomial to obtain upper bounds on $\operatorname{gr}(L)$ for alternating links with 16 or fewer crossings.

\subsection{Definition of girth and properties}

Recall that $G_{+}(D)$ is the graph obtained from the all-positive Kauffman state of diagram $D$ (see Section 2.4), and that the girth $\ell\left(G_{+}(D)\right)$ is the length of the shortest cycle in this graph.

Definition 26. The girth of link $L$ is given by $\operatorname{gr}(L)=\max \left\{\ell\left(G_{+}(D)\right) \mid D\right.$ is a diagram of $\left.L\right\}$.

Theorem 31. Let $L$ be a prime alternating link. If $D, D^{\prime}$ are two reduced alternating diagrams of $L$, then $G_{+}(D), G_{+}\left(D^{\prime}\right)$ have the same girth.

Proof. The Tait flyping conjecture states that any two reduced alternating diagrams of $L$ are related by flypes [MT91]. Flypes may be expressed as a series of mutations, which induce Whitney flips on the corresponding graph [Gre11]. Thus $G_{+}(D)$ and $G_{+}\left(D^{\prime}\right)$ are 2-isomorphic graphs. Girth is an invariant of the cycle matroid [Oxl92], so $G_{+}(D)$ and $G_{+}\left(D^{\prime}\right)$ have the same girth.

For a specific alternating diagram, crossing information gives upper bounds on the girths of $G_{+}(D)$ and $G_{-}(D)$, the graphs related to the all-positive and all-negative Kauffman states $s_{+}(D)$ 
and $s_{-}(D)$ (see Section 2.4). Recall that $\sigma(L)$ is a link invariant given by the signature of the Seifert matrix obtained from any diagram of $L$. We denote the number of crossings in a diagram by $c(D)$ and the numbers of positive and negative crossings by $c_{+}(D), c_{-}(D)$ respectively.

Theorem 32. Let $L$ be a nontrivial, non-split alternating link, and let $D$ be a reduced alternating diagram of $L$. Then $\ell\left(G_{+}(D)\right) \leq \frac{2 c(D)}{c_{+}(D)+\sigma(L)+2}$ and $\ell\left(G_{-}(D)\right) \leq \frac{2 c(D)}{c_{-}(D)-\sigma(L)+2}$.

Proof. The graph $G_{+}(D)$ is planar and connected, so the girth $\ell\left(G_{+}(D)\right)$ is related to the numbers of edges and vertices by the following inequality: $E \leq \frac{\ell}{\ell-2}(v-2)$ (see e.g. [Die00]). Since $D$ is reduced, we may assume that $\ell \geq 2$ and rearrange the inequality as $\ell \leq \frac{2 E}{E-v+2}$. The number of edges $E$ is the number of crossings $c(D)$, and the number of vertices is the number of connected components $s_{+}$in the all-positive smoothing of $D$. Using the formula $\sigma(L)=s_{+}(D)-c_{+}(D)-1$ ([Tra04]) we obtain the first inequality above. Similarly, $\sigma(L)=-s_{-}(D)+c_{-}(D)+1$ gives an upper bound for the girth of $G_{-}(D)$.

As a corollary of Theorem 25 in Section 3.4, if Khovanov and chromatic homology agree on 3 or more gradings, this agreement imposes a restriction on the type of graphs that realize the isomorphism.

Corollary 17. Suppose that link L is homologically thin with Jones polynomial:

$$
J_{L}(q)=\beta_{0} q^{C}+\beta_{1} q^{C+2}+\beta_{2} q^{C+4}+\beta_{3} q^{C+6}+\ldots
$$

and that $D$ is a diagram of $L$ whose all-positive state graph $G$ has girth $\ell>2$. Then the cyclomatic number of $G$ is equal to $-\beta_{1}$ and the number of $\ell$-cycles in $G$ is equal to $\left(\begin{array}{c}-\beta_{1}-1+(\ell-1) \\ \ell-1\end{array}\right)-\left|\beta_{\ell}\right|$.

The 2nd coefficient of $J(L)$ uniquely determines the first $\operatorname{gr}(L)$ coefficients. This leads to a somewhat surprising result.

Theorem 33. Suppose that link $L$ is homologically thin with $\operatorname{gr}(L)=G \geq 3$. If $D$ is a diagram of $L$, the all-positive state graph associated to D must have girth equal to 1, 2, or $G$.

Proof. The result is trivial for $G=3$, so we consider $G>3$. Using the notation of Corollary 17, we know that $\beta_{i}=(-1)^{i}\left(\begin{array}{c}-\beta_{1}+i-1 \\ i\end{array}\right)$ for $0 \leq i<G-1$ and $\beta_{G-1}=(-1)^{G-1}\left(\left(\begin{array}{c}-\beta_{1}+(G-1)-1 \\ G-1\end{array}\right)-n_{G}\right)$, where $n_{G}$ is the number of $G$-cycles in any all-positive state graph graph with girth $G$ (Theorem 25, Corollary 17).

Now suppose that there exists a diagram $D$ of $L$ such that $G_{+}(D)$ has girth $H$, with $3 \leq H<$ $G$. As above, Theorem 25 implies that $\left.\beta_{H-1}=(-1)^{H-1}\left(\begin{array}{c}-\beta_{1}+(H-1)-1 \\ H-1\end{array}\right)-n_{H}\right)$ where $n_{H}$ is the 
number of $H$-cycles in $G_{+}(D)$. Since $H-1<G-1$, we compare the formulas for $\beta_{H-1}$ given by the equations above:

$$
(-1)^{H-1}\left(\begin{array}{c}
-\beta_{1}+(H-1)-1 \\
H-1
\end{array}\right)=\beta_{H-1}=(-1)^{H-1}\left(\left(\begin{array}{c}
-\beta_{1}+(H-1)-1 \\
H-1
\end{array}\right)-n_{H}\right) .
$$

This implies that $n_{H}$ is equal to zero, which is a contradiction since $G_{+}(D)$ must have at least one cycle of order $H$.

We can find lower bounds for $\operatorname{gr}(L)$ by constructing diagrams with favorable properties, as in the following results.

Corollary 18. The girth of the positive $T(2, n)$ torus knot is equal to $n$, and the girth of its mirror image is equal to 2.

Proof. The Jones polynomial of $T(2, n)$ can be written in the form $\sum_{k=1}^{n-1}(-1)^{k} q^{-(n+1)-2 k}-q^{-(n-1)}$ [KSU09]. The coefficients of the Jones polynomial are $\beta_{i}=1$ for $i$ even, $0 \leq i<n-1$ and $\beta_{i}=-1$ for $i$ odd, $0<i<n-1$ as well as $i=n-1$. The first $n-1$ coefficients alternate between 1 and -1 , so these could be the coefficients of the chromatic polynomial of a graph whose girth is $n$ and whose cyclomatic number is $p_{1}=1$ (see Theorem 25). Since $\beta_{n-2}=\beta_{n-1}=-1$, the girth cannot be any greater than $n$. The standard $n$-twist diagram demonstrates equality (see Figure 2.7 in Section $2.4)$.

For the mirror image of $T(2, n)$, the Jones polynomial has the same coefficients in reverse order. After changing signs so that $\beta_{0}=1$ according to our convention, the first two coefficients are both equal to 1 . Since no graph has negative cyclomatic number, Theorem 25 implies the girth of this knot is less than 3 . The mirror of the standard $n$-twist diagram yields a girth of 2 .

Corollary 19. Let $L$ be an alternating pretzel link with twist parameters $\left(-a_{1},-a_{2}, \ldots,-a_{n}\right)$. Then $\operatorname{gr}(L) \geq \min \left\{a_{i}+a_{j} \mid 1 \leq i \neq j \leq n\right\}$.

Proof. Draw the diagram of $L$ which has $n$ columns of left-hand-twists (see Section 3.2.3).

The connected sum of two oriented knots $K_{1}, K_{2}$ is well-defined for any choice of planar diagrams for these two knots. There is a lower bound for the girth of the connected sum $K_{1} \# K_{2}$ in terms of the girths of the summands.

Theorem 34. $\operatorname{gr}\left(K_{1} \# K_{2}\right) \geq \min \left\{g r\left(K_{1}\right), g r\left(K_{2}\right)\right\}$.

Proof. Let $D_{1}$ be a diagram of $K_{1}, D_{2}$ be a diagram of $K_{2}$ such that $\ell\left(G_{+}\left(D_{1}\right)\right)=g r\left(K_{1}\right)$ and $\ell\left(G_{+}\left(D_{2}\right)\right)=g r\left(K_{2}\right)$. When we perform the connected sum operation on $D_{1}$ and $D_{2}$, the all-positive state graph of the new diagram consists of $G_{+}\left(D_{1}\right)$ and $G_{+}\left(D_{2}\right)$ joined at a single vertex, with girth $\min \left\{\operatorname{gr}\left(K_{1}\right), \operatorname{gr}\left(K_{2}\right)\right\}$. 


\subsection{Khovanov homology bound on girth}

In this section, we show that as the girth of a graph goes to infinity, so does the span of chromatic homology and also the corresponding part of Khovanov homology. For any graph $G$, let $v$ be the number of vertices in $G$ and $b$ the number of blocks.

Lemma 4. Let $M$ be the maximum cycle length in a connected graph $G$. Then $b \leq v-M+1$.

Proof. Suppose there exists such a graph $G$ with $b>v-M+1$ or, equivalently, $b-1>v-M$. By assumption, there is a cycle of length $M$ in $G$, call it $P_{M}$. The number of vertices in the set $V(G) \backslash V\left(P_{M}\right)$ is $v-M$, and the number of blocks in $G$ that do not contain $P_{M}$ is $b-1$. Each vertex in $V(G) \backslash V\left(P_{M}\right)$ can contribute at most one additional block to $G$; i.e., $v-M \geq b-1$. But this contradicts our initial assumption.

Lemma 4 holds true if we replace $M$ with the length of any cycle in $G$, including the girth. We will use the inequality with $\ell(G)$ to prove Theorem 35 .

Theorem 35. The homological span of chromatic homology hspan $\left(H_{\mathcal{A}_{m}}(G)\right)$ goes to infinity as the girth $\ell(G)$ goes to infinity.

Proof. By Theorem 26 and Lemma 4: $\operatorname{hspan}\left(H_{\mathcal{A}_{m}}(G)\right) \geq v-b \geq v-(v-\ell+1)=\ell-1$.

Theorem 36. The homological span of Khovanov homology hspan $(K h(L))$ goes to infinity as the girth $\operatorname{gr}(L)$ goes to infinity.

As a corollary, we get that the girth of any link can not be infinite, since we know the span of Khovanov homology.

Theorem 37. The girth $\operatorname{gr}(L)$ of any link $L$ is finite.

In particular, the span of Khovanov homology provides an upper bound on girth of a link. In the case where Khovanov homology of a knot is thick, the number of non-trivial $i$-gradings before homology becomes thick gives an upper bound on girth of $L$, since chromatic homology is always thin.

Example 11 (Family of links with arbitrarily large girth). Consider the mirror of the 12-crossing non-alternating knot $12 n 888$ [CL17, LS17] shown in Figure 5.1(a) and denoted $\overline{12 n 888}$. The Khovanov homology of this knot has minimal homological grading $i=-12$. The homological width of $K h(\overline{12 n 888})$ is three but the homology is supported on two diagonals for $-12 \leq i<-5$, where the width increases to 3 diagonals. This implies that the girth of $\overline{12 n 888}$ lies in the range $3 \leq \operatorname{gr}(\overline{12 n 888}) \leq 7$. 


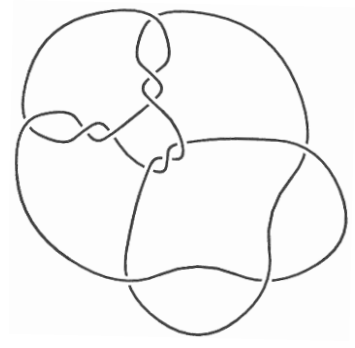

(a)

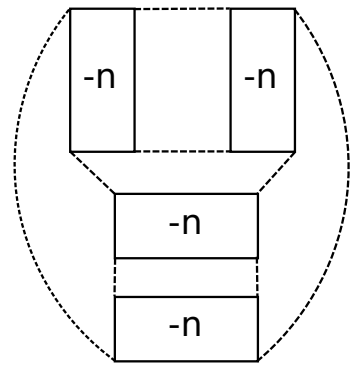

(c)

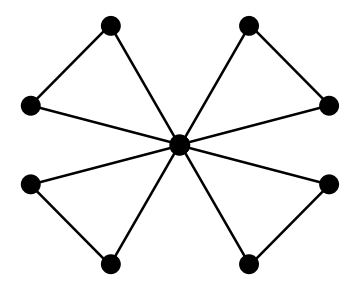

(b)

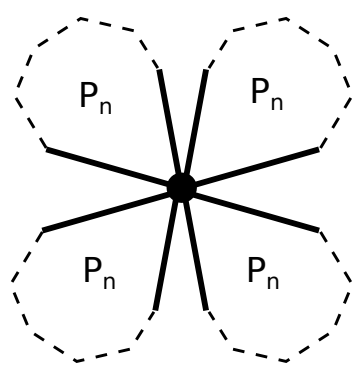

(d)

Figure 5.1: (a) Mirror of the link 12n888; (b) Graph $G_{+}\left(D_{3}\right)$ corresponding to diagram in (a); (c) Infinite family $D_{n}=\overline{-(n ; n)(n ; n)}$; (d) Graph $G_{+}\left(D_{n}\right)$ corresponding to diagram in (c).

The Conway notation for the standard diagram of $12 n 888$ is $-(3 ; 3)(3 ; 3)$ [CL17]. Let $D_{3}=$ $\overline{-(3 ; 3)(3 ; 3)}$ be the diagram corresponding to the mirror of $12 n 888$. The graph $G_{+}\left(D_{3}\right)$ consists of four triangles joined at a single vertex; see Figure 5.1(b).

Let $L D_{n}$ denote a link determined by diagram $D_{n}=\overline{-(n ; n)(n ; n)}$ obtained from $D_{3}$ by simultaneously increasing the number of twists corresponding to each parameter in Conway symbol [JSO7]; see Figure 5.1(c). The family of graphs associated to these diagrams consists of vertex gluing of four $n$-gons $G_{+}\left(D_{n}\right)=P_{n} * P_{n} * P_{n} * P_{n}$; see Figure 5.1(d). Thus the girth $\ell\left(G_{+}\left(D_{n}\right)\right)=n$ and the range of homological degrees where the isomorphism of Theorem 7 holds goes to infinity as $n$ increases. However, the Khovanov homology of these links $L D_{n}$ is thick with much larger span, and we can only describe a portion of the thin part. Tables 5.1 and 5.2 contain partial computations for Khovanov homology of $L D_{4}$ and chromatic homology of $G_{+}\left(D_{4}\right)=P_{4} * P_{4} * P_{4} * P_{4}$ with boldface entries denoting matching homology groups. 
Table 5.1: Khovanov homology of the link $L D_{4}=\overline{-(4 ; 4)(4 ; 4)}$ with boldface entries denoting matching homology with chromatic homology.

\begin{tabular}{|c|c|c|c|c|c|c|c|c|c|}
\hline \multirow{2}{*}{\multicolumn{2}{|c|}{$K h^{p, q}\left(D_{4}\right)$}} & \multicolumn{8}{|c|}{$\mathbf{p}$} \\
\hline & & \multirow{2}{*}{-16} & \multirow{2}{*}{-15} & \multirow{2}{*}{-14} & \multirow{2}{*}{-13} & \multirow[t]{2}{*}{-12} & \multirow[t]{2}{*}{-11} & \multirow{2}{*}{$\begin{array}{c}-10 \\
.\end{array}$} & \multirow{2}{*}{ 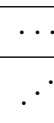 } \\
\hline \multirow{8}{*}{ q } & $\vdots$ & & & & & & & & \\
\hline & -33 & & & & & & $\mathbb{Z}^{13}$ & $\mathbb{Z}^{15} \oplus \mathbb{Z}_{2}^{15}$ & \\
\hline & -35 & & & & & $\mathbb{Z}^{10}$ & $\mathbb{Z}^{15} \oplus \mathbb{Z}_{2}^{13}$ & & \\
\hline & -37 & & & & $\mathbb{Z}^{6}$ & $\mathbb{Z}^{13} \oplus \mathbb{Z}_{2}^{10}$ & & & \\
\hline & -39 & & & $\mathbb{Z}^{4}$ & $\mathbb{Z}^{10} \oplus \mathbb{Z}_{2}^{6}$ & & & & \\
\hline & -41 & & & $\mathbb{Z}^{6} \oplus \mathbb{Z}_{2}^{4}$ & & & & & \\
\hline & -43 & $\mathbb{Z}$ & $\mathbb{Z}^{4}$ & & & & & & \\
\hline & -45 & $\mathbb{Z}$ & & & & & & & \\
\hline
\end{tabular}

Table 5.2: Chromatic homology of $G=G_{+}\left(D_{4}\right)=P_{4} * P_{4} * P_{4} * P_{4}$ with boldface entries denoting matching homology with chromatic homology.

\begin{tabular}{|c|c|c|c|c|c|c|c|}
\hline \multirow{2}{*}{$H_{\mathcal{A}_{2}}^{i, j}(G)$} & \multicolumn{6}{|c|}{ i } \\
\cline { 2 - 8 } & $\mathbf{0}$ & $\mathbf{1}$ & $\mathbf{2}$ & $\mathbf{3}$ & $\mathbf{4}$ & $\cdots$ \\
\hline $\mathbf{1 3}$ & $\mathbb{Z}$ & & & & & \\
\hline $\mathbf{1 2}$ & $\mathbb{Z}$ & $\mathbb{Z}^{4}$ & & & & \\
\hline & $\mathbf{1 1}$ & & & $\mathbb{Z}^{6} \oplus \mathbb{Z}_{2}^{4}$ & & & \\
\cline { 2 - 8 } $\mathbf{j}$ & $\mathbf{1 0}$ & & & $\mathbb{Z}^{4}$ & $\mathbb{Z}^{10} \oplus \mathbb{Z}_{2}^{6}$ & & \\
\cline { 2 - 8 } & $\mathbf{9}$ & & & & $\mathbb{Z}^{6}$ & $\mathbb{Z}^{9} \oplus \mathbb{Z}_{2}^{10}$ & \\
\cline { 2 - 7 } & $\mathbf{8}$ & & & & & $\mathbb{Z}^{10}$ & $\ddots$ \\
\hline & $\vdots$ & & & & & & $\ddots$ \\
\hline
\end{tabular}

\subsection{Computational results}

The description of the Jones coefficients in Theorem 25 provides an upper bound for the girth of homologically thin links. If the first $n-1$ coefficients follow the pattern $1,-b,\left(\begin{array}{c}b+1 \\ 2\end{array}\right),-\left(\begin{array}{c}b+2 \\ 3\end{array}\right), \ldots$ for some $b \in \mathbb{Z}_{\geq 0}$, but the $n$th coefficient is not equal to $(-1)^{n}\left(\begin{array}{c}b-1+n \\ n\end{array}\right)$, then we know that $g r(L)$ cannot be greater than $n$. Using Mathematica and the KnotTheory package [Kat], we computed the Jones polynomials and determined these upper bounds for all prime alternating knots (and their mirror images) with crossing number less than or equal to 16. The results are displayed in Table 5.3. 
Table 5.3: Upper bounds for girth of alternating knots (up to 16 crossings), based on Jones polynomial coefficients.

\begin{tabular}{|c|c|c|c|c|c|c|c|c|c|c|c|c|c|c|c|}
\hline & \multicolumn{14}{|c|}{ Upper bound for $g r(K), K$ alternating } \\
\hline & & 2 & 3 & 4 & 5 & 6 & 7 & 8 & 9 & 10 & 11 & 12 & 13 & 14 & 15 \\
\hline & $\leq 10$ & 4 & 271 & 76 & 29 & 8 & 3 & 0 & 1 & 0 & 0 & 0 & 0 & 0 & 0 \\
\hline & 11 & 1 & 566 & 108 & 51 & 5 & 2 & 0 & 0 & 0 & 1 & 0 & 0 & 0 & 0 \\
\hline & 12 & 0 & 1949 & 503 & 99 & 21 & 4 & 0 & 0 & 0 & 0 & 0 & 0 & 0 & 0 \\
\hline$c(K)$ & 13 & 1 & 7580 & 1555 & 569 & 47 & 2 & 1 & 0 & 0 & 0 & 0 & 1 & 0 & 0 \\
\hline & 14 & 0 & 30681 & 6629 & 1656 & 97 & 7 & 1 & 1 & 0 & 0 & 0 & 0 & 0 & 0 \\
\hline & 15 & 1 & 133803 & 29736 & 6681 & 291 & 10 & 2 & 0 & 1 & 0 & 0 & 0 & 0 & 1 \\
\hline & 16 & 0 & 607412 & 119148 & 32131 & 876 & 25 & 2 & 3 & 1 & 0 & 0 & 0 & 0 & 0 \\
\hline
\end{tabular}

Note that for knots with odd crossing numbers in Table 5.3, there is a single knot for which the upper bound on girth is equal to the minimal crossing number, and a single knot for which the upper bound is equal to 2 . These are the positive $(2, n)$ torus link and its mirror, respectively. After 11 crossings, the distribution of the upper bounds seems to stabilize, with the proportion of knots with girth 2, 3, or 4 staying consistently close to 95 percent of the total. The girth of an alternating knot will never be equal to 1 , because any alternating knot has a reduced diagram. 


\section{Chapter 6}

\section{Future directions}

Here we list some open problems directly related to our results and some of the methods we will use to attack them, organized by topic.

\subsection{Chromatic graph homology over $\mathcal{A}_{2}$}

Through results about the structure of chromatic homology over $\mathcal{A}_{2}$, we can prove statements about Khovanov homology and the Jones polynomial. Future work may build on results in Chapter 3 to describe how various operations on graphs affect their chromatic homology. This investigation might lead to simpler proofs of chromatic polynomial results and allow us to explicitly describe Khovanov homology for links with certain families of diagrams.

We would like to investigate how torsion in Khovanov homology is related to classical link invariants and topological properties of links. It is interesting to note that the reduced version of the standard Khovanov homology has very little torsion, and none at all for knots with fewer than 13 crossings. However, the reduced odd version may have torsion of order 3 or higher for relatively small knots [Shu11]. These computations suggest that some torsion may arise from algebraic considerations rather than the topological properties of a link.

The coefficients of the Jones polynomial give an upper bound for the girth of a link. We would like to determine whether every link has a diagram whose all-positive state graph realizes the maximum possible girth. For specific families of links, results about the Jones polynomial (e.g., [Lan98] for pretzel links) may provide new bounds or explicit formulas for girth. At present, Theorems 25 and 33 are stated only for homologically thin links. These results may potentially be generalized by showing that there are no generators for Khovanov homology in the lowest quantum gradings, so that the head of the Jones polynomial is determined entirely by gradings which correspond to chromatic homology. Our approach will utilize a spanning tree model for Khovanov homology [Weh08]. 


\subsection{Chromatic graph homology over $\mathcal{A}_{m}, m>2$}

The general form of the tail (last nontrivial homological grading) of chromatic homology over $\mathcal{A}_{m}$ is still unknown. In Section 4.2, we conjecture that the tail consists of some number of copies of the tail of a cycle $P_{m}$, and that this number may vary depending on the structural properties of the graph. In future work, we will find a precise characterization of the final grading and use it to describe the homological span (Conjecture 1).

Other properties of chromatic homology do not have an explicit combinatorial description. For instance, while the width of chromatic homology is known over all values of $m$ (Theorem 27), we do not have an analogous formula for the width of torsion. In [PPS09], part of the chromatic homology of graph $G$ over $\mathcal{A}_{3}$ is described using a cell complex that depends on the interactions between cycles of $G$. There may be similar geometric realizations that describe a larger part of chromatic homology.

General constructions for pairs of non-isomorphic graphs with the same chromatic polynomial are described in [Chi88, DKT05]. It may be possible to distinguish all pairs generated by these constructions using chromatic homology over $\mathcal{A}_{3}$. Chromatic homology over $\mathcal{A}_{3}$ is stronger than the $\mathcal{A}_{2}$ version, but we do not know the relative strengths of chromatic homology for $m>2$. For instance, we are not aware of any examples which show whether $\mathcal{A}_{5}$ is stronger than $\mathcal{A}_{3}$. Future work will determine whether such relationships exist and whether torsion is needed to distinguish cochromatic graphs over $\mathcal{A}_{m}$. 


\section{REFERENCES}

[AP04] Marta Asaeda and Jozef Przytycki. Khovanov homology: torsion and thickness. In Advances in Topological Quantum Field Theory, volume 179 of NATO Science Series II, pages 135-166. Springer, 2004.

[Bal11] Jonathan Baldwin. On the spectral sequence from Khovanov homology to Heegaard Floer homology. International Mathematics Research Notices, 15:3426-3470, 2011.

[Bar02] Curtis Barefoot. Block-cutvertex trees and block-cutvertex partitions. Discrete Mathematics, 256(1-2):35-54, 2002.

[Bie05] Halina Bielak. A new method for counting chromatic coefficients. Annales UMCS Informatica AI, 3(1):179-189, 2005.

[BM76] John Bondy and Uppaluri Murty. Graph theory with applications. Elsevier, 1976.

[BN02] Dror Bar-Natan. On Khovanov's categorification of the Jones polynomial. Algebraic and Geometric Topology, 2:337-370, 2002.

[Bol98] Béla Bollobás. Modern Graph Theory. Graduate Texts in Mathematics. Springer, 1998.

[BS15] Joshua Batson and Cotton Seed. A link-splitting spectral sequence in Khovanov homology. Duke Mathematical Journal, 164(5):801-841, 2015.

[CCR08] Michael Chmutov, Sergei Chmutov, and Yongwu Rong. Knight move in chromatic cohomology. European Journal of Combinatorics, 29(1):311-321, 2008.

[Chi88] Gek Chia. Some remarks on the chromatic uniqueness of graphs. Ars Combinatoria, 26A:65-72, 1988.

[CL85] Chong-Yun Chao and Nian-Zu Li. On trees of polygons. Archiv der Mathematik, 45(2):180-185, 1985. 
[CL17] Jae Choon Cha and Charles Livingston. KnotInfo: Table of Knot Invariants. http://www.indiana.edu/ knotinfo/, July 27, 2017.

[CWJ79] Chong-Yun Chao and Earl Whitehead Jr. Chromatically unique graphs. Discrete Mathematics, 27(2):171-177, 1979.

$\left[\mathrm{DHK}^{+} 04\right]$ Fengming Dong, Michael Hendy, Khee Meng Koh, Charles Little, and Kie Leong Teo. Chromatically unique multibridge graphs. Electronic Journal of Combinatorics, 11(1), 2004.

[Die00] Reinhard Diestel. Graph Theory. Graduate Texts in Mathematics. Springer, 2000.

[DKT05] Fengming Dong, Khee Meng Koh, and Kie Leong Teo. Chromatic Polynomials and Chromaticity of Graphs. World Scientific, Singapore, 2005.

[DL06] Oliver Dasbach and Xiao-Song Lin. On the head and tail of the colored Jones polynomial. Compositio Mathematica, 142(2):1332-1342, 2006.

[dMN05] Anna de Mier and Mark Noy. On matroids determined by their Tutte polynomials. Discrete Mathematics, 302(1-3):52-76, 2005.

[EH07] Michael Eastwood and Stephen Huggett. Euler characteristics and chromatic polynomials. European Journal of Combinatorics, 28(6):1553-1560, 2007.

[Far80] Edward Farrell. On chromatic coefficients. Discrete Mathematics, 29:257-264, 1980.

[Gre10] Joshua Greene. Homologically thin, non-quasi-alternating links. Mathematical Research Letters, 17(1):39-49, 2010.

[Gre11] Joshua Greene. Conway mutation and alternating links. In Proceedings of Gökova Geometry-Topology Conference 2011, pages 31-41, 2011.

[Hav] Frédéric Havet. Combinatorial optimization - algorithms for telecommunications. http://www-sop.inria.fr/members/Frederic.Havet/Cours/ubinet.html. 
[HGPR06] Laure Helme-Guizon, Jozef Przytycki, and Yongwu Rong. Torsion in graph homology. Fundamenta Mathematicae, 190:139-177, 2006.

[HGR05] Laure Helme-Guizon and Yongwu Rong. A categorification for the chromatic polynomial. Algebraic and Geometric Topology, 5:1365-1388, 2005.

[HGR12] Laure Helme-Guizon and Yongwu Rong. Khovanov type homologies for graphs. Kobe Journal of Mathematics, 29(1-2):25-43, 2012. URL: https://arxiv.org/pdf/math/ 0506023.pdf.

[Hoc45] Gerhard Hochschild. On the cohomology groups of an associative algebra. Annals of Mathematics, 46:58-67, 1945.

[HP66] Frank Harary and Geert Prins. The block-cutpoint-tree of a graph. Publicationes Mathematicae Debrecen, 13:103-107, 1966.

[HW14] Richard Hepworth and Simon Willerton. Categorifying the magnitude of a graph. Homology, Homotopy and Applications, 16(2):1-30, 2014.

[JHR06] Edna Jasso-Hernandez and Yongwu Rong. A categorification for the Tutte polynomial. Algebraic and Geometric Topology, 6(5):2031-2049, 2006.

[JS07] Slavik Jablan and Radmila Sazdanović. LinKnot: Knot Theory by Computer. World Scientific, 2007.

[Kat] The Knot Atlas. http://katlas.org.

[Kau11] Louis Kauffman. Khovanov homology. arXiv:1107.1524 [math.GT], 2011.

[KG90] Khee Meng Koh and Bee Hua Goh. Two classes of chromatically unique graphs. Discrete Mathematics, 82(1):13-24, 1990.

[Kho00] Mikhail Khovanov. A categorification of the Jones polynomial. Duke Mathematical Journal, 101(3):359-426, 2000. 
[Kho03] Mikhail Khovanov. Patterns in knot cohomology, I. Experimental Mathematics, $12(3): 365-374,2003$.

[KM11] Peter Kronheimer and Tomasz Mrowka. Khovanov homology is an unknot detector. Publications mathématiques de l'IHÉS, 113(1):97-208, 2011.

[KR08a] Mikhail Khovanov and Lev Rozansky. Matrix factorizations and link homology. Fundamenta Mathematicae, 199(1):1-91, 2008.

[KR08b] Mikhail Khovanov and Lev Rozansky. Matrix factorizations and link homology ii. Geometry and Topology, 12(3):1387-1425, 2008.

[KSU09] Abdullah Kopuzlu, Abdulgani Sahin, and Tamer Ugur. On polynomials of $K(2, n)$ torus knots. Applied Mathematical Sciences, 3(59):2899-2910, 2009.

[Lan98] Ryan Landvoy. The Jones polynomial of pretzel knots and links. Topology and its Applications, 83:135-147, 1998.

[Lee05] Eun Soo Lee. An endomorphism of the Khovanov invariant. Advances in Mathematics, 197(2):554-586, 2005.

[Lic97] William Lickorish. An introduction to knot theory. Graduate Texts in Mathematics. Springer, 1997.

[Lod98] John-Louis Loday. Cyclic homology, volume 301 of Grundlehren der mathematischen Wissenschaften. Springer, 2nd edition, 1998.

[LR11] Kerry Luse and Yongwu Rong. A categorification for the Penrose polynomial. Journal of Knot Theory and its Ramifications, 20(1):141-157, 2011.

[LS17] Adam Lowrance and Radmila Sazdanović. Khovanov homology, chromatic homology, and torsion. Topology and its Applications, 222:77-99, 2017.

[Man13] Andrew Manion. The Khovanov homology of 3-strand pretzels, revisited. arXiv:1303.3303 [math.GT], 2013. 
[Man14] Andrew Manion. The rational Khovanov homology of 3-strand pretzel links. Journal of Knot Theory and its Ramifications, 23(8), 2014.

[Mer72] Guy Meredith. Coefficients of chromatic polynomials. Journal of Combinatorial Theory, Series B, 13:14-17, 1972 .

$\left[\mathrm{MPS}^{+} 17\right]$ Sujoy Mukherjee, Jozef Przytycki, Marithania Silvero, Xiao Wang, and Seung Yeop Yang. Search for torsion in Khovanov homology. Experimental Mathematics, 2017.

[MT91] William Menasco and Morwen Thistlethwaite. The Tait flyping conjecture. Bulletin of the American Mathematical Society, 25(2):403-412, 1991.

[Ng05] Lenhard Ng. A Legendrian Thurston-Bennequin bound from Khovanov homology. Algebraic and Geometric Topology, 5:1637-1653, 2005.

[ORS13] Peter Oszváth, Jacob Rasmussen, and Zoltán Szabó. Odd Khovanov homology. Algebraic and Geometric Topology, 13:1465-1488, 2013.

[OS04] Peter Oszváth and Zoltán Szabó. Holomorphic disks and knot invariants. Advances in Mathematics, 186(1):58-116, 2004.

[OS05] Peter Oszváth and Zoltán Szabó. On the Heegaard Floer homology of branched doublecovers. Advances in Mathematics, 194(1):1-33, 2005.

[OS08] Peter Oszváth and Zoltán Szabó. On the Khovanov and Knot Floer homologies of quasialternating links. Proceedings of the Gökova Geometry-Topology Conference 2007. International Press of Boston, 2008.

[Ox192] James Oxley. Matroid Theory. Oxford University Press, New York, 1992.

[PPS09] Milena Pabiniak, Jozef Przytycki, and Radmila Sazdanović. On the first group of the chromatic cohomology of graphs. Geometriae Dedicata, 140(1):19-48, 2009.

[Prz10] Jozef Przytycki. When the theories meet: Khovanov homology as Hochschild homology of links. Quantum Topology, 1(2):93-109, 2010. 
[PS14] Jozef Przytycki and Radmila Sazdanović. Torsion in Khovanov homology of semiadequate links. Fundamenta Mathematicae, 225:277-303, 2014.

[PS16] Krzysztof Putyra and Alexander Shumakovitch. Knot invariants arising from homological operations on Khovanov homology. J. of Knot Theory and Ram., 25(3), 2016.

[Qaz11] Khaled Qazaqzeh. The Khovanov homology of a family of three-column pretzel links. Communications in Contemporary Mathematics, 13(5), 2011.

[Ras10] Jacob Rasmussen. Khovanov homology and the slice genus. Inventiones Mathematicae, 182(2):419-447, 2010.

[Rea68] Ronald Read. An introduction to chromatic polynomials. Journal of Combinatorial Theory, 4:52-71, 1968.

[Rol03] Dale Rolfsen. Knots and links. AMS Chelsea, 2003.

[Sco] Daniel Scofield. URL: https://danscofield.wordpress.ncsu.edu/files/2018/03/ graphhomology-A5.pdf.

[Shu11] Alexander Shumakovitch. Patterns in odd Khovanov homology. Journal of Knot Theory and its Ramifications, 20(1):203-222, 2011.

[Shu14] Alexander Shumakovitch. Torsion of Khovanov homology. Fundamenta Mathematicae, 225(0):343-364, 2014.

[Shu16] Alexander Shumakovitch. Torsion in Khovanov homology of homologically thin knots. Forthcoming, 2016.

[Sta12] Laura Starkston. The Khovanov homology of $(p,-p, q)$ pretzel knots. Journal of Knot Theory and its Ramifications, 21(5), 2012.

[Sto08] Marko Stosić. Categorification of the dichromatic polynomial for graphs. Journal of Knot Theory and its Ramifications, 17(31):31-45, 2008. 
[SY18] Radmila Sazdanović and Martha Yip. A categorification of the chromatic symmetric function. Journal of Combinatorial Theory, Series A, 154:218-246, 2018.

[Sys79] Maciej Syslo. Characterizations of outerplanar graphs. Discrete Mathematics, 26:47-53, 1979.

[Tra04] Pawel Traczyk. A combinatorial formula for the signature of alternating diagrams. Fundamenta Mathematicae, 184:311-316, 2004.

[Tut54] William Tutte. A contribution to the theory of chromatic polynomials. Canadian Journal of Mathematics, 6:80-91, 1954.

[Vir04] Oleg Viro. Khovanov homology, its definitions and ramifications. Fundamenta Mathematicae, 184:317-342, 2004.

[Weh08] Stephan Wehrli. A spanning tree model for Khovanov homology. Journal of Knot Theory and its Ramifications, 17(12):1561-1574, 2008.

[Whi35] Hassler Whitney. On the abstract properties of linear dependence. American Journal of Mathematics, 57(3):509-533, 1935.

[Wit12] Edward Witten. Khovanov homology and gauge theory. Geometry and Topology Monographs, 18:291-308, 2012.

[WJZ84] Earl Whitehead Jr. and Lian-Chang Zhao. Cutpoints and the chromatic polynomial. Journal of Graph Theory, 8(3):371-377, 1984.

[WW92] Christopher Wakelin and Douglas Woodall. Chromatic polynomials, polygon trees, and outerplanar graphs. Journal of Graph Theory, 16(5):459-466, 1992.

[Zyk49] Aleksandr Zykov. On some properties of linear complexes. Matematicheskii Sbornik, 24(66):163-188, 1949. 
APPENDIX 


\section{Appendix A}

\section{Proofs of combinatorial identities}

The proof of Theorem 25 transforms the coefficients of chromatic polynomial to the coefficients of the Jones polynomial. Two steps in the proof require the use of combinatorial identities which are not immediately obvious and whose justification would interrupt the flow of the text.

Proposition 10. $\sum_{i=0}^{n}(-1)^{i}\left(\begin{array}{l}x \\ i\end{array}\right)\left(\begin{array}{c}y-i \\ y-n\end{array}\right)=(-1)^{n}\left(\begin{array}{c}x-y-1+n \\ n\end{array}\right)$.

Proof. We will use the formula $\left(\begin{array}{l}a \\ b\end{array}\right)=(-1)^{a-b}\left(\begin{array}{c}-(b+1) \\ a-b\end{array}\right)$ as well as the Chu-Vandermonde identity $\sum_{k=0}^{n}\left(\begin{array}{l}a \\ k\end{array}\right)\left(\begin{array}{c}b \\ n-k\end{array}\right)=\left(\begin{array}{c}a+b \\ n\end{array}\right)$.

$$
\begin{aligned}
\sum_{i=0}^{n}(-1)^{i}\left(\begin{array}{c}
x \\
i
\end{array}\right)\left(\begin{array}{c}
y-i \\
y-n
\end{array}\right) & =\sum_{i=0}^{n}(-1)^{i}\left(\begin{array}{l}
x \\
i
\end{array}\right)(-1)^{n-i}\left(\begin{array}{c}
-(y-n+1) \\
n-i
\end{array}\right) \\
& =(-1)^{n} \sum_{i=0}^{n}\left(\begin{array}{c}
x \\
i
\end{array}\right)\left(\begin{array}{c}
-(y-n+1) \\
n-i
\end{array}\right) \\
& =(-1)^{n}\left(\begin{array}{c}
x-y+n-1 \\
n
\end{array}\right) .
\end{aligned}
$$

Proposition 11. $\sum_{i=0}^{n}\left(\begin{array}{c}x-2+i \\ i\end{array}\right)=\left(\begin{array}{c}x-1+n \\ n\end{array}\right)$.

Proof. For any positive integers $a, b$, we have $\left(\begin{array}{c}a-1 \\ b-1\end{array}\right)+\left(\begin{array}{c}a-1 \\ b\end{array}\right)=\left(\begin{array}{l}a \\ b\end{array}\right)$, and so $\left(\begin{array}{l}a \\ b\end{array}\right)-\left(\begin{array}{c}a-1 \\ b\end{array}\right)=\left(\begin{array}{l}a-1 \\ b-1\end{array}\right)$. We take the difference of the two quantities above and apply this identity $n$ times to show that the 
result is equal to zero.

$$
\begin{aligned}
\left(\begin{array}{c}
x-1+n \\
n
\end{array}\right) & -\sum_{i=0}^{n}\left(\begin{array}{c}
x-2+i \\
i
\end{array}\right) \\
& =\left(\begin{array}{c}
x-1+n \\
n
\end{array}\right)-\left(\left(\begin{array}{c}
x-2+n \\
n
\end{array}\right)+\left(\begin{array}{c}
x-2+(n-1) \\
n-1
\end{array}\right)+\ldots+\left(\begin{array}{c}
x-2 \\
0
\end{array}\right)\right) \\
& =\left(\begin{array}{c}
x-1+n \\
n
\end{array}\right)-\left(\begin{array}{c}
x-2+n \\
n
\end{array}\right)-\left(\left(\begin{array}{c}
x-2+(n-1) \\
n-1
\end{array}\right)+\ldots+\left(\begin{array}{c}
x-2 \\
0
\end{array}\right)\right) \\
& =\left(\begin{array}{c}
x-2+n \\
n-1
\end{array}\right)-\left(\left(\begin{array}{c}
x-2+(n-1) \\
n-1
\end{array}\right)+\left(\begin{array}{c}
x-2+(n-2) \\
n-2
\end{array}\right)+\ldots+\left(\begin{array}{c}
x-2 \\
0
\end{array}\right)\right) \\
& =\left(\begin{array}{c}
x-2+n \\
n-1
\end{array}\right)-\left(\begin{array}{c}
x-2+(n-1) \\
n-1
\end{array}\right)-\left(\left(\begin{array}{c}
x-2+(n-2) \\
n-2
\end{array}\right)+\ldots+\left(\begin{array}{c}
x-2 \\
0
\end{array}\right)\right) \\
& \ldots\left(\begin{array}{c}
x-1-n+n \\
0
\end{array}\right)-\left(\begin{array}{c}
x-2 \\
0
\end{array}\right)=\left(\begin{array}{c}
x-1 \\
0
\end{array}\right)-\left(\begin{array}{c}
x-2 \\
0
\end{array}\right)=0 .
\end{aligned}
$$

